\title{
T-branes at the limits of geometry
}

\author{
Lara B. Anderson, ${ }^{a}$ Jonathan J. Heckman, ${ }^{b}$ Sheldon Katz $^{c}$ and Laura P. Schaposnik ${ }^{d}$ \\ ${ }^{a}$ Physics Department, Robeson Hall, Virginia Tech, \\ 850 West Campus Drive, Blacksburg, VA 24061, U.S.A. \\ ${ }^{b}$ Department of Physics, University of North Carolina, \\ 120 E Cameron Ave, Chapel Hill, NC 27599, U.S.A. \\ ${ }^{c}$ Department of Mathematics, University of Illinois at Urbana-Champaign, \\ 1409 W. Green Street, Urbana, IL 61801, U.S.A. \\ ${ }^{d}$ Department of Mathematics, University of Illinois at Chicago, \\ 851 S. Morgan Street, Chicago, IL 60607, U.S.A. \\ E-mail: lara.anderson@vt.edu, jheckman@email.unc.edu, \\ katzs@illinois.edu, schapos@uic.edu
}

ABSTRACT: Singular limits of 6D F-theory compactifications are often captured by Tbranes, namely a non-abelian configuration of intersecting 7-branes with a nilpotent matrix of normal deformations. The long distance approximation of such 7-branes is a Hitchin-like system in which simple and irregular poles emerge at marked points of the geometry. When multiple matter fields localize at the same point in the geometry, the associated Higgs field can exhibit irregular behavior, namely poles of order greater than one. This provides a geometric mechanism to engineer wild Higgs bundles. Physical constraints such as anomaly cancellation and consistent coupling to gravity also limit the order of such poles. Using this geometric formulation, we unify seemingly different wild Hitchin systems in a single framework in which orders of poles become adjustable parameters dictated by tuning gauge singlet moduli of the F-theory model.

Keywords: F-Theory, Differential and Algebraic Geometry, Superstring Vacua

ARXiv EPRINT: 1702.06137 


\section{Contents}

1 Introduction $\quad 1$

2 T-branes on a curve $\quad 6$

2.1 Nilpotent nucleation $\quad 7$

2.1.1 Heterotic dual 8

3 T-branes at a simple point $\quad 10$

3.1 Minimal nilpotent orbits: classical algebras 12

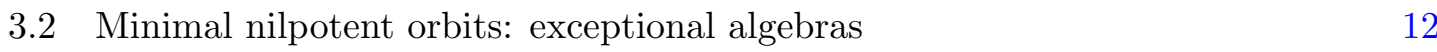

4 T-branes gone wild $\quad 13$

$\begin{array}{lll}4.1 & \text { Wild matter } & 15\end{array}$

$\begin{array}{ll}\text { 4.1.1 Coordinate free formulation } & 19\end{array}$

$\begin{array}{lll}4.2 & \text { Moduli spaces } & 22\end{array}$

5 Geometric unification $\quad 24$

5.1 A wild compact SU(2) model 26

5.2 Tangent bundle to $K 3$ revisited 29

6 Conclusions $\quad 30$

A Introduction to wild Hitchin systems

B An integrable system $\quad 43$

C Complex structure deformations $\quad 45$

C.1 Unfolding $A_{2} \quad 50$

\section{Introduction}

There is a close interplay between the geometry of extra dimensions in string theory and low energy effective field theory. In a theory of open and closed strings it is common to associate geometry with closed string modes such as the graviton, and field theory sectors with open string modes. This leads to a physically rich space of vacua.

An important goal in string compactification is to characterize all resulting effective field theories. One general lesson is that the open and closed string sectors often provide complementary pictures, much as one would assign coordinate patches on a manifold. Celebrated examples include open/closed string channel duality, and the AdS/CFT correspondence [1]. A priori, however, there is no reason to expect a single patch to cover 
all regimes. From this perspective, the important question is to determine the transition functions required to move from one patch to another.

This is particularly pressing in F-theory, where the backreaction of 7-branes on the 10D spacetime is encoded in terms of an auxiliary 12D geometry given by a torus fibration over the 10D spacetime. This "closed string" perspective is quite helpful in determining how to consistently couple 7-branes to gravity. In this approach, one also encounters singular regions in the $10 \mathrm{D}$ spacetime where the torus fibration degenerates. In such situations, the geometric picture breaks down, and one instead passes to the gauge theory on a 7-brane, namely the open string sector.

But in general the moduli space of the 7-brane gauge theory will contain more than just classical commutative geometry. This is because the degrees of freedom for 7-branes are captured by matrix degrees of freedom. As such, typically only the eigenvalues of a matrix translate into commutative geometry. When the matrix degrees of freedom do not commute, we pass to a more general configuration in which the 7-brane puffs up in directions transverse to its worldvolume. This is known as a T-brane [2,3], as the matrix of normal deformations is upper triangular. For recent work on the formal structure of T-branes in F-theory, see e.g. [4-8]. For phenomenological applications of T-branes, see e.g. [3, 9-15]. For reviews on F-theory model building, see e.g. [16-20].

In this paper we study T-brane vacua for $6 \mathrm{D}$ and $4 \mathrm{D}$ theories with eight real supercharges. More precisely, we consider F-theory compactified on an elliptically fibered Calabi-Yau threefold. This yields a $6 \mathrm{D}$ theory with $\mathcal{N}=(1,0)$ supersymmetry. Further compactification on a $T^{2}$ yields a $4 \mathrm{D} \mathcal{N}=2$ theory which we can alternatively study using type IIA string theory compactified on the same Calabi-Yau threefold. Our goal will be to take steps towards a general prescription for the limiting behavior of T-branes as we pass from the "open string patch" of moduli space to the "closed string patch" captured by Calabi-Yau geometry.

This point of view can lead to a sharp correspondence between the moduli spaces of a Hitchin system [21] on a Riemann surface $C$ with gauge group $G$ of ADE type, and the local Calabi-Yau threefold $X$ associated with a curve of ADE singularities [4, 22, 23]. Recall that in the Hitchin system we have an adjoint valued $(1,0)$ form $\Phi$ and a gauge connection $A$. Gauge invariant Casimir invariants of $\Phi$ translate in the type IIA CalabiYau geometry to complex structure deformations, while periods of the gauge field (more precisely its holonomies) translate to periods of the Ramond-Ramond three-form potential, with values in the intermediate Jacobian $H^{3}(X, \mathbb{R}) / H^{3}(X, \mathbb{Z})$. The transition between these two descriptions of patches of moduli space is captured by the theory of limiting mixed Hodge structures [4]. There a global/compact description of T-branes was proposed that described the Hitchin moduli space (open string degrees of freedom) as "emergent" in a singular limit of the CY geometry. The precise correspondence between Hitchin and 
singular CY moduli was laid out in [4] and can be summarized by the following diagram

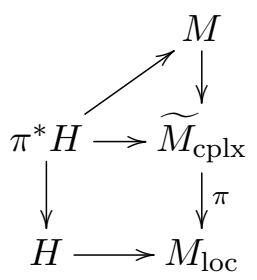

where $H$ and $M$ are the full Hitchin and Calabi-Yau moduli spaces, respectively, and $\widetilde{M}_{\text {cplx }}$ and $M_{l o c}$ the complex structure moduli spaces of the resolved Calabi-Yau geometry and local (singularity preserving) complex structure deformations of the singular Calabi-Yau geometry (note: the bottom map is the Hitchin fibration and the top map is an inclusion).

This prompts a number of natural questions:

- Does this correspondence extend to singular field configurations of the Hitchin system? And in what singular Calabi-Yau geometries might these arise?

- Does F-theory impose physical constraints on such singularities?

While we will not fully resolve these questions in the present work, our aim in this paper will be to show that to a large extent, there is a natural extension to the case of Higgs fields with singularities, which again can lead to a perfect match between open and closed string moduli. In addition, we will also show that the overall type of singularities which can be engineered are often constrained by the further condition that a compact F-theory or type IIA background really exists.

In physical terms, the Hitchin system on a Riemann surface $C$ emerges as the long distance description of 7-branes wrapped on $C$. It is specified by introducing a gauge group $G$, an adjoint valued $(1,0)$-form $\Phi$, and a gauge field $A$. Solutions to the equations of motion at generic points of $C$ are [21]:

$$
\bar{\partial}_{A} \Phi=0 \text { and } F+\left[\Phi, \Phi^{\dagger}\right]=0,
$$

modulo gauge transformations. The correspondence between the moduli of this system and the associated local curve of ADE singularities has been studied in references [4, 2224]. T-Branes correspond to the special class of configurations where $\Phi$ is nilpotent in the Lie algebra. For a matrix valued $\Phi$, i.e., for the classical algebras, this amounts to the condition $\operatorname{Tr}\left(\Phi^{l}\right)=0$ for sufficiently large $l$. As the moduli space of the Hitchin system (with smooth Higgs field) is connected, there is a sense in which we can build up quite general solutions starting from a T-brane configuration. Indeed, starting from such a configuration, we can perform perturbations in the entries of this solution, thus realizing a broad class of additional solutions [25]. In the dual frame of heterotic string constructions, this is the statement that there is a single connected component to the moduli space of stable holomorphic vector bundles on a $K 3$ surface.

Now, in actual physical applications, we also expect to have matter fields localized at points of the geometry. In F-theory, these matter fields are realized from collisions of 


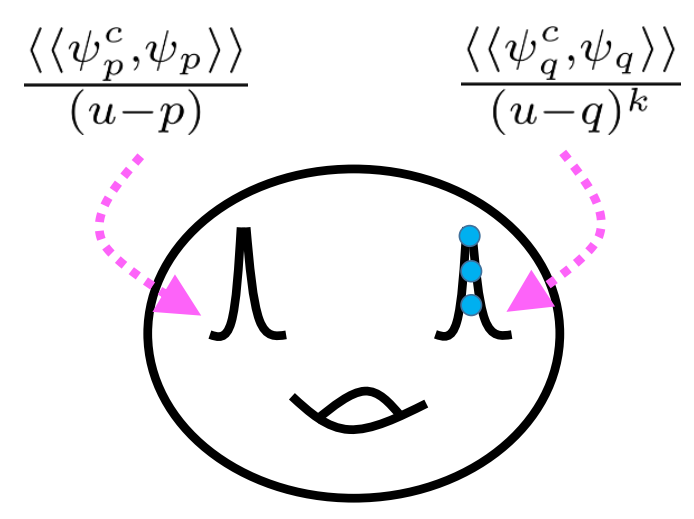

Figure 1. Depiction of a Hitchin system on a genus one curve with poles at marked points indicated by narrow cylindrical regions, that is, spikes. Background values for localized matter fields induce poles in the Higgs field of the Hitchin system. On the left we depict matter localized at $u=p$ which generates a simple pole. On the right we depict matter localized at the non-reduced scheme $(u-q)^{k}=0$ which generates a higher order pole.

intersecting 7-branes. Background values for these fields lead to localized sources for the Hitchin system equations. This is reflected in singularities (including possibly higher order singularities) for the Higgs field:

$$
\Phi \sim d u\left(\frac{T_{k}}{u^{k}}+\ldots+\frac{T_{1}}{u}\right)
$$

where $T_{i}$ are elements of the complexified algebra, and the singularity is concentrated at $u=0$, with $u$ a local coordinate on the curve. The case of a simple pole, namely $k=1$ has been studied in various contexts, and is known in the Hitchin system literature as a regular singularity. This case is particularly tractable because the data of the singularity is fully captured by a residue around $u=0$. Higher order poles are often referred to as irregular or wild. This case is more delicate because a residue will fail to detect higher order terms. T-brane configurations correspond to cases where any or all of the $T_{i}$ are actually nilpotent.

Physical considerations impose limits on possible T-brane phenomena. Such constraints arise because the total number of matter fields in a string compactification often obeys additional conditions beyond those imposed by the local Hitchin system. In this paper we provide a physical picture from string compactification for such singularities. Moreover, we will show the sense in which the structure of possible singularities is constrained by the additional assumption that they arise from a genuine string compactification.

The essential point is that these singularities are all induced from background values for localized matter fields. The case of higher order singularities involves a particular subtlety in F-theory compactification which as far as we are aware, has not been addressed previously. Much as in earlier work on localized matter in F-theory (see e.g. [3, 24, 26]), we consider a Hitchin system with gauge group $G_{\text {parent }}$. Activating a background value for the Higgs field $\Phi_{\text {parent }}$ initiates a breaking pattern to a lower rank gauge group $G$, with matter fields in various irreducible representations of $G$. Schematically, localized matter 
fields are associated with elements in the ring:

$$
\frac{\mathbb{C}[u]}{\left(\alpha_{R}(u)\right)}
$$

where $\alpha_{R}(u)$ serves to remind us that the localization depends on the choice of representation $R$ for a given matter field. Now, in the generic case, $\alpha_{R}$ has a simple zero, and this corresponds to the case of a single localized matter field. When we have higher order zeros, we obtain additional matter fields localized at the same point. This is possible because strictly speaking, there is a difference between the point defined by $u=0$ and that defined by $u^{k}=0$. In the latter case, we have what is sometimes referred to as a non-reduced scheme. Additional structure lurks in such objects, which as we argue is crucial in developing a consistent picture for how localized matter appears in F-theory compactifications. Indeed, background values for these higher order matter fields translate to higher order poles for the Higgs field of the theory with gauge group $G$. Varying these background values then determines a moduli space of vacua which we match to that of a wild Hitchin system. See figure 1 for a depiction of higher order poles induced from background values for matter fields.

In some sense, this accomplishes the main point of matching open and closed string moduli. Indeed, since expectation values for localized matter correspond in F-theory to complex structure deformations of the Calabi-Yau, we see that the limiting behavior of these moduli (in tandem with the rest of the intermediate Jacobian) provide a characterization which extends to wild Hitchin systems as well.

Physical considerations impose additional constraints. In 6D vacua, anomaly cancellation conditions tend to impose tight restrictions on the total number of matter fields. This in turn limits the possible order of poles which can be realized in a Hitchin system derived from intersecting 7 -branes. In $4 \mathrm{D} \mathcal{N}=2$ vacua, the related anomaly cancellation condition is quite innocuous, but is instead replaced by the stringent condition that gravity can be consistently decoupled.

On the other hand, the geometric formulation of these emergent Hitchin-like systems provides a single framework to unify seemingly different moduli space problems. Indeed, we can pass to wild Higgs bundle configurations with different orders for poles simply by adjusting the gauge singlet moduli of the F-theory model.

To test these ideas further, we also present some examples of compact F-theory geometries which realize the above considerations. In particular, we focus on the case of an SU(2) Hitchin system with poles. An additional feature of these global examples is that there can often be multiple higher order singularities which must all be treated simultaneously in the Hitchin system. Tracking through the different possible singularity types for the Hitchin system and their geometric avatars reveals a precise match.

The rest of this paper is organized as follows. In section 2 we review some of the previous work on T-branes in F-theory and type IIA compactifications on an elliptically fibered Calabi-Yau threefold. We also extend some of these results, explaining how Tbranes can be used as a nucleation point for building more general solutions to the Hitchin system, and consequently, the class of geometries realized by such configurations. After this, in section 3 we turn to the case of T-branes at a simple point, namely cases where 
the Higgs field develops a simple pole. In section 4 we turn to the case of Higgs fields with higher order singularities. We derive the main equations for the Higgs field in these cases, determine the structure of the moduli space, and explain the sense in which physics imposes non-trivial constraints on the order of poles in the system. We follow this with explicit compact models in section 5. We present our conclusions and future directions for research in section 6 . In the appendices we provide additional mathematical details on the formal structure of wild Hitchin systems and the correspondence with geometry.

\section{T-branes on a curve}

In this section we review the realization of T-branes on a curve in the Hitchin system, and in particular, how this data can be mapped to the local moduli of a Calabi-Yau threefold defined by a curve of singularities. This correspondence actually appears in two related physical contexts. First of all, we can consider $6 \mathrm{D}$ supersymmetric vacua generated by F-theory on an elliptically fibered Calabi-Yau threefold. The Hitchin system on a curve $C$ comes about as the long distance approximation for 7-branes wrapped on $C$. As already mentioned, this yields a system with $\mathcal{N}=(1,0)$ supersymmetry, i.e. eight real supercharges. Compactifying on an additional $T^{2}$, we obtain type IIA string theory on the same CalabiYau threefold, and a $4 \mathrm{D} \mathcal{N}=2$ supersymmetric effective field theory.

Consider, then, F-theory on an elliptically fibered Calabi-Yau threefold $X$ with base $B$. In minimal Weierstrass form, we have:

$$
y^{2}=x^{3}+f x+g
$$

where $f$ and $g$ are sections of $\mathcal{O}_{B}\left(-4 K_{B}\right)$ and $\mathcal{O}_{B}\left(-6 K_{B}\right)$, with $K_{B}$ the canonical class of the base $B$. For each irreducible component $C$ of the discriminant $\Delta=4 f^{3}+27 g^{2}$ we get a 7-brane gauge theory with gauge group $G$, as dictated by the order of vanishing for $f$ and $g$, as well as possible monodromic identifications in the fiber. The field content which propagates on $C$ includes an adjoint valued $(1,0)$ form $\Phi$, and a gauge connection $A$. When the background values of all localized matter fields are zero, the equations of motion for this system are [21]:

$$
\bar{\partial}_{A} \Phi=0 \text { and } F+\left[\Phi, \Phi^{\dagger}\right]=0,
$$

modulo unitary gauge transformations:

$$
\Phi \mapsto g^{\dagger} \Phi g \text { and } A \mapsto g^{\dagger} A g+g^{\dagger} \bar{\partial} g
$$

We can parameterize the moduli space of solutions using gauge invariant Casimir invariants constructed from $\Phi$. This yields the base of the Hitchin system moduli space. For example, in the case of an $\mathrm{SU}(N)$ gauge theory, take $\operatorname{Tr}\left(\Phi^{j}\right)$ for $j=2, \ldots, N$. The full hyperkahler moduli space is then filled out by also specifying the holonomies of the gauge field $A$ along one-cycles of $C$.

In the match to Calabi-Yau geometry, the base of the Hitchin system maps to the local complex structure moduli, that is, those moduli which can deform the singularity type of 
a local curve of singularities. ${ }^{1}$ The fiber of the moduli space is, in IIA language given by the $\mathrm{RR}$ moduli filling out periods in $H^{3}(X, \mathbb{R}) / H^{3}(X, \mathbb{Z})$, the intermediate Jacobian. A T-brane configuration corresponds to the special case where $\Phi$ is nilpotent over all of $C$. This is clearly a rather special set of conditions to satisfy.

The match between Hitchin space degrees of freedom and localized moduli has nontrivial implications for Calabi-Yau geometry. For example, since the moduli space of the Hitchin system (with smooth Higgs field) consists of a single connected component, we can perform a small perturbation in such a configuration to reach one in which $\Phi$ is not nilpotent. One way to establish the existence of a single connected component is to work in terms of the complexified connection $\mathcal{A}=A+\Phi+\Phi^{\dagger}$ with curvature $\mathcal{F}$ so that the Hitchin system equations become [21]:

$$
\mathcal{F}=0
$$

The existence of the match with Calabi-Yau moduli, in tandem with the existence of a single connected component means it is enough to take limiting behavior in the CalabiYau complex structure moduli to produce simple T-branes of the Hitchin system. In future sections we will consider the extension of some of these results to the case of Hitchin sytems with singularities and the associated T-branes.

\subsection{Nilpotent nucleation}

Even though such nilpotent configurations are quite special, they provide a convenient way to generate a broad class of explicit solutions to the Hitchin system. To illustrate, we will construct below T-branes in 6D theories which are never physically "rigid" (i.e. forming an isolated component of moduli space). Instead, starting from a nilpotent solution, we can perturb to more general configurations.

We construct a T-brane by holding fixed a nilpotent element $\mu$ of the complexified Lie algebra $\mathfrak{g}_{\mathbb{C}}$. At the level of group theory, by a theorem of Jacobson and Morozov there exists a homomorphism

$$
\rho_{\mu}: \mathfrak{s l}(2, \mathbb{C}) \rightarrow \mathfrak{g}_{\mathbb{C}}
$$

taking the raising operator of $\mathfrak{s l}(2, \mathbb{C})$ to $\mu$. In general, the image of this $\mathfrak{s l}(2, \mathbb{C})$ will take values in a maximal subalgebra $\mathfrak{h}_{\mathbb{C}}$ such that its commutant $\mathfrak{c}_{\mathbb{C}}$ is the "unbroken" gauge symmetry. Using this, we can construct T-branes in two steps:

- First, construct a solution in the nilpotent cone for the $\mathrm{SU}(2)$ Hitchin system.

- Second, define a map from the $\mathrm{SU}(2)$ Hitchin system to the Hitchin system with gauge group $G$ induced by the homomorphism $\rho_{\mu}$.

A general theorem of Hitchin [25] ensures that there is a corresponding solution to the Hitchin system with gauge symmetry $\mathfrak{h}_{\mathbb{C}}$. So, starting from a T-brane configuration, we sweep out a local neighborhood in the moduli space.

\footnotetext{
${ }^{1}$ For some discussion of the extension of this correspondence to the case of Calabi-Yau fourfolds, see e.g. $[24,27,28]$.
} 
In [29], more general moduli spaces of nilpotent SU(2) Higgs fields were constructed in which the Higgs fields were allowed to vanish at isolated points. Even more generally, the above construction of T-branes can be extended to higher rank gauge groups whose Higgs fields can take values in nonzero nilpotent orbits of smaller dimension at isolated points. For example, an SU(3) T-brane on a curve can be constructed whose Higgs field has rank 2 at the generic point of the curve, has rank 1 at isolated points, and vanishes at another set of isolated points.

\subsubsection{Heterotic dual}

It is also instructive to study the structure of this 'nucleation' in the heterotic dual. Recall that in 6D Heterotic / F-theory duality, stable holomorphic vector bundles on an elliptically fibered K3 surface correspond to elliptically fibered Calabi-Yau threefolds with base a Hirzebruch surface $\mathbb{F}_{n}$ with $-12 \leq n \leq 12 .{ }^{2}$ From this perspective, we would like to verify that starting from a stable holomorphic vector bundle $V$ with structure group SU(2), we can construct a holomorphic vector bundle with more general structure group $G \subset E_{8}$. In particular, we wish to verify that there are deformation moduli available which can connect this solution to one with generic values of complex structure in the associated spectral cover. Phrased differently, we will use embeddings of the bundle structure group $\mathrm{SU}(2) \subset E_{8}$ to probe $^{3}$ the general moduli space of $G$-bundles over $K 3$.

Assuming a particular embedding of $\mathrm{SU}(2) \rightarrow E_{8}$, the adjoint representation of $E_{8}$ will decompose into various symmetric powers of the fundamental representation of SU(2). These additional representations specify smoothing deformations which take us from the original embedding of the vector bundle to a more general vector bundle with structure group $G$. It should be noted that the exact structure group obtainable will be determined by $c_{2}(V)$ and a complete description of those $G$-bundle moduli spaces here is beyond the scope of the present work. It would be interesting to fully classify this structure in future work (see e.g. [33] for more details on the possible moduli spaces). We now establish that some such smoothing deformations always exist. Said differently, our aim is to count the number of zero modes associated with various symmetric powers of $S^{j} V$.

Since we are assuming $V$ is a stable vector bundle with a non-trivial instanton number, we have that:

$$
\int_{K 3} c_{2}(V)=12+n
$$

where $-8 \leq n \leq 12$. The reason for the lower bound -8 is that we are assuming we can performing a breaking pattern down to $E_{7}$. For supersymmetric vacua there is also an upper bound of at most 24 instantons. As $V$ is stable, we also have:

$$
h^{0}\left(K 3, S^{j} V\right)=h^{2}\left(K 3, S^{j} V\right)=0 \quad \text { for } \quad j>0 .
$$

\footnotetext{
${ }^{2}$ We are using the standard convention of $\mathrm{F}$-theory whereby $\mathbb{F}_{n}$ means $\mathbb{F}_{-n}$ if $n<0$. This convention is made to distinguish between the situations where the section of $\mathbb{F}_{n}$ on which a gauge group is placed has positive or negative self-intersection.

${ }^{3}$ Note that the notion of using simple bundles as a "probe" of more general bundle moduli spaces has also been successfully employed in 4D heterotic compactifications [30-32].
} 
As a consequence, the index theorem counts all the zero modes coming from $S^{j} V$ :

$$
-h^{1}\left(K 3, S^{j} V\right)=\int_{K 3} \operatorname{ch}\left(S^{j} V\right) \operatorname{Td}(K 3)=\operatorname{rk}\left(S^{j} V\right) \chi\left(K 3, \mathcal{O}_{K 3}\right)+\int_{K 3} \operatorname{ch}_{2}\left(S^{j} V\right)
$$

or:

$$
-h^{1}\left(K 3, S^{j} V\right)=2(j+1)+\int_{K 3} \operatorname{ch}_{2}\left(S^{j} V\right) .
$$

Our task therefore reduces to calculating the second Chern character of $S^{j} V$. Here, we use the splitting principle. For some line bundle $L$ on $K 3$, we have, for $k>0$ an integer:

$$
\begin{aligned}
\operatorname{ch}_{2}(V) & =\operatorname{ch}_{2}\left(L \oplus L^{-1}\right) \\
\operatorname{ch}_{2}\left(S^{2 k} V\right) & =\operatorname{ch}_{2}\left(L^{2 k} \oplus L^{2 k-2} \ldots \oplus \mathcal{O}_{K 3} \oplus \ldots \oplus L^{2-2 k} \oplus L^{-2 k}\right) \\
& =\operatorname{ch}_{2}\left(L^{2 k}\right)+\operatorname{ch}_{2}\left(L^{2 k-2}\right)+\ldots+\operatorname{ch}_{2}\left(L^{2-2 k}\right)+\operatorname{ch}_{2}\left(L^{-2 k}\right) \\
\operatorname{ch}_{2}\left(S^{2 k+1} V\right) & =\operatorname{ch}_{2}\left(L^{2 k+1} \oplus L^{2 k-1} \ldots \oplus L \oplus L^{-1} \oplus \ldots \oplus L^{1-2 k} \oplus L^{-2 k-1}\right) \\
& =\operatorname{ch}_{2}\left(L^{2 k+1}\right)+\operatorname{ch}_{2}\left(L^{2 k-1}\right)+\ldots+\operatorname{ch}_{2}\left(L^{1-2 k}\right)+\operatorname{ch}_{2}\left(L^{-2 k-1}\right) .
\end{aligned}
$$

In other words, we get:

$$
\operatorname{ch}_{2}\left(S^{2 k} V\right)=\sum_{m=1}^{k} 4 m^{2} c_{1}(L)^{2}=\frac{2(2 k+1)(k+1) k}{3} c_{1}(L)^{2} .
$$

Returning to our computation of the dimension $h^{1}\left(K 3, S^{j} V\right)$ therefore yields:

$$
-h^{1}\left(K 3, S^{2 k} V\right)=2(2 k+1)+\frac{2(2 k+1)(k+1) k}{3} \int_{K 3} c_{1}(L)^{2} .
$$

On the other hand, we also know that the instanton number of the vector bundle is set by:

$$
\int_{K 3} c_{1}(L)^{2}=\int_{K 3} \operatorname{ch}_{2}(V)=-\int_{K 3} c_{2}(V)=-(12+n) .
$$

Hence, we get:

$$
h^{1}\left(K 3, S^{2 k} V\right)=\left(\frac{2 k+1}{3}\right)(2 k(k+1)(12+n)-6) .
$$

Similarly, for odd symmetric powers we get

$$
\operatorname{ch}_{2}\left(S^{2 k+1} V\right)=\sum_{m=0}^{k}(2 m+1)^{2} c_{1}(L)^{2}=\frac{(2 k+3)(2 k+1)(k+1)}{3} c_{1}(L)^{2},
$$

which leads to

$$
h^{1}\left(K 3, S^{2 k+1} V\right)=\left(\frac{k+1}{3}\right)((2 k+3)(2 k+1)(12+n)-12)
$$

as above.

The important point for us is that the deformation moduli of our vacuum configuration have $j>1$, and thus in particular we always have $S^{2} V=\operatorname{End}_{0}(V)$. As $j=2$ sets a lower bound for the dimension $h^{1}\left(K 3, S^{j} V\right)$, we have:

$$
h^{1}\left(K 3, S^{j} V\right) \geq 42+4 n>0
$$


where in the rightmost inequality we used the fact that $n \geq-8$. So, we always have deformation moduli available to move us back to a non-singular configuration (in the language of the F-theory dual geometry). Note that this result implies that for the case of F-theory duals of such heterotic models, the moduli space of the induced singular Hitchin systems can also be connected (see $[4,34]$ for examples of such dual heterotic/F-theory pairs).

\section{T-branes at a simple point}

In the previous section we focused on the case of T-brane phenomena for a genus $g$ Riemann surface. Now, in physical realizations, it is also quite common that the fields of the Hitchin system may develop singularities at points of this Riemann surface. To illustrate, observe that without any such singularities, $\Phi$ is an adjoint valued $(1,0)$ form, so the Casimir invariants $\operatorname{Tr}\left(\Phi^{n}\right)$ will be holomorphic sections of the bundle $K_{C}^{n}$, i.e. elements in $H^{0}\left(C, K_{C}^{n}\right)$. On the other hand, it is also common in physical applications for the Riemann surface to be a $\mathbb{P}^{1}$ for which $H^{0}\left(C, K_{C}^{n}\right)=0$. In these cases, the Hitchin system becomes non-trivial via the fact that a $(1,0)$-form or a higher differential on $\mathbb{P}^{1}$ can develop poles at various points of the curve. This is in fact a general occurrence in F-theory. Examples include elliptically fibered Calabi-Yau threefolds with base a Hirzebruch surface.

Now, another closely related feature of physical models is the presence of matter localized at points of the geometry. In the context of $6 \mathrm{D}$ theories, these matter fields fill out $6 \mathrm{D}$ hypermultiplets which transform in some representation $R$ of the gauge group $G$. When the representation is pseudo-real, it is also possible to have half hypermultiplets. For a hypermultiplet, we have a pair of scalars $\psi \oplus \psi^{c}$, where the first scalar transforms in the representation $R$ and the second transforms in the conjugate (i.e., dual) representation $R^{c}$. In the associated Calabi-Yau geometry, localized matter fields are often interpreted near the collision of distinct components of the discriminant locus. ${ }^{4}$

There is a close interplay between the background values for these hypermultiplet scalars and possible polar terms in the Higgs field. Indeed, the holomorphic F-term data of the Hitchin system now receives the correction term (see e.g. [24]):

$$
\bar{\partial}_{A} \Phi=\delta_{p}\left\langle\left\langle\psi^{c}, \psi\right\rangle\right\rangle
$$

where $\delta_{p}$ is a delta function (namely, a $(1,1)$ current) localized at the point $u=p$. Here, we have also introduced the canonical pairing with image in the adjoint representation of the complexified algebra:

$$
\langle\langle\cdot, \cdot\rangle\rangle: R^{c} \otimes R \rightarrow \operatorname{ad}\left(\mathfrak{g}_{\mathbb{C}}\right) .
$$

In the context of colliding 7-branes, it can happen that there are actually multiple hypermultiplets all concentrated at the same point. For example, in the collision of an $\operatorname{SU}(N)$ 7-brane with an $\mathrm{SU}(M)$ 7-brane, the hypermultiplets transform in the bifundamental representation $(\mathbf{N}, \overline{\mathbf{M}})$, so from the perspective of the $\mathrm{SU}(N)$ gauge theory we actually have $M$ hypermultiplets in the fundamental representation of $\mathrm{SU}(N)$. Let us also note that it is

\footnotetext{
${ }^{4}$ Of course, this presupposes that geometry is an accurate guide to the matter spectrum, a point which can be obscured by T-branes [3]!
} 
not even necessary to have weakly coupled matter fields. Strongly coupled generalizations of such hypermultiplets known as conformal matter generate the same sort of deformations of the Hitchin system [35-38]. In this more general formulation, we simply have a source term sitting on the right hand side of equation (3.1). Assuming such a source term is present, and denoting by "..." the regular terms, integrating equation (3.1) yields:

$$
\Phi \sim d u \frac{\left\langle\left\langle\psi^{c}, \psi\right\rangle\right\rangle}{u-p}+\ldots
$$

In physical constructions, one typically has multiple marked points, each with localized matter. When this matter has a non-zero background value, we obtain a parabolic Higgs bundle. See appendix A for review of some aspects of this case. ${ }^{5}$ Holding fixed a choice of boundary conditions at each such marked point, we can then construct a corresponding moduli space for the Hitchin system. Here, the gauge invariant data of the boundary condition is captured by the conjugacy class in $\mathfrak{g}_{\mathbb{C}}$ of the residue. Of course, in the full physical construction we are free to vary the background values of the hypermultiplets $\psi \oplus \psi^{c}$, and in so doing change the boundary conditions for the parabolic Higgs bundle.

For a given choice of background fields at a marked point, we obtain a nilpotent element $\mu \in \mathfrak{g}_{\mathbb{C}}$. The conjugacy class is then specified by the nilpotent orbit of this element. This non-zero background value also initiates a breaking pattern of $\mathfrak{g}_{\mathbb{C}}$ to a commutant subalgebra which we denote by $\mathfrak{c}_{\mathbb{C}}$. Roughly speaking, the more hypermultiplets with non-zero background values, the lower the rank of $\mathfrak{c}_{\mathbb{C}}$. The precise breaking pattern of course depends on the specific representations in question, and is best addressed using the Bala-Carter theory of nilpotent orbits (see e.g. [40]).

This data is hidden from the complex structure of the local Calabi-Yau geometry. Just as in reference [4], we can start from a nilpotent element, and the corresponding raising operator $T_{+}$of the associated $\mathfrak{s u}(2)$ subalgebra. Perturbing by the lowering operator $T_{-}=T_{+}^{\dagger},{ }^{6}$ we obtain a family of diagonalizable deformations:

$$
T(\varepsilon)=T_{+}+\varepsilon T_{-} .
$$

An interesting feature of this procedure is that the closure of the conjugacy class can indeed jump between the cases $\varepsilon=0$ and $\varepsilon \neq 0$. Let us note that examples of this type include minimal rigid nilpotent orbits. Such boundary conditions are important in the context of geometric Langlands duality and rigid surface operators [41].

Indeed, if we are only interested in the parabolic Hitchin system, each choice of conjugacy class labels a distinct component of the moduli space. Physically, however, we recognize that these different choices of boundary conditions are connected to one another by activating background values of localized matter [24]. One can view the results of the present paper as a general method for geometrically engineering various surface operators, but in which we extend the moduli space by promoting some boundary conditions to dynamical fields.

\footnotetext{
${ }^{5}$ For recent work on parahoric Hitchin systems and the corresponding integrable systems, see [39].

${ }^{6}$ Strictly speaking, this perturbation as we have described it may only make sense locally along the curve.
} 
Our plan in the rest of this section will be to present some examples of T-branes at a simple point. In particular, we shall focus on the case of minimal nilpotent orbits, namely those cases where the commutant subalgebra has maximal rank. For all simple algebras other than $\mathfrak{e}_{8}$, this is realized via a non-zero background value for a single hypermultiplet in the fundamental representation of the algebra. In the case of $\mathfrak{e}_{8}$, the analogue of localized matter fields is instead played by conformal matter fields, namely, the Higgs branch of heterotic small instantons. We revisit this example in subsection 5.2.

\subsection{Minimal nilpotent orbits: classical algebras}

To illustrate the general idea, we begin by constructing the minimal nilpotent orbits when the gauge group $G$ is a simple classical algebra, namely the cases of the $\mathrm{SU}(N), \operatorname{Sp}(2 N)$ and $\mathrm{SO}(2 N)$ algebras. Since the latter two cases arise in string constructions from adding orientifolds and/or monodromic quotients to the $\mathrm{SU}(N)$ case, we shall primarily confine our discussion to the geometric realization of minimal nilpotent $\mathrm{SU}(N)$ algebras.

Recall that we are interested in constructing a T-brane configuration such that the commutant subalgebra has maximal rank. The relevant decomposition into subalgebras is:

$$
\begin{aligned}
\mathfrak{s u}(N) & \supset \mathfrak{s u}(2) \times \mathfrak{s u}(N-2) \times \mathfrak{u}(1) \\
\mathfrak{s p}(2 N) & \supset \mathfrak{s u}(2) \times \mathfrak{s p}(2 N-2) \\
\mathfrak{s o}(2 N) & \supset \mathfrak{s u}(2) \times \mathfrak{s o}(2 N-4) \times \mathfrak{u}(1) \\
\mathfrak{s o}(2 N+1) & \supset \mathfrak{s u}(2) \times \mathfrak{s} \mathfrak{o}(2 N-3) \times \mathfrak{u}(1),
\end{aligned}
$$

where the T-brane is embedded in the $\mathfrak{s u}(2)$ factor. Referring back to equation (3.4), let us note that for all cases other than the $\mathfrak{s u}(N)$ example, the closure of the conjugacy classes for $\varepsilon=0$ and $\varepsilon \neq 0$ are different.

We shall now turn to the geometric realization of these deformations, at least for the case $\varepsilon \neq 0$. For ease of exposition, we focus on the case of the $\mathfrak{s u}$ algebra. Similar considerations hold for the other cases, using for example the spectral curve of the associated Hitchin system. The local presentation of the Calabi-Yau threefold is given by a curve of A-type singularities. We can write this as:

$$
y^{2}=x^{2}+u^{N}+\varepsilon u^{N-2} .
$$

We realize a T-brane by taking a limit with $\varepsilon \rightarrow 0$. The analysis of this case is rather similar to what is presented in reference [4].

\subsection{Minimal nilpotent orbits: exceptional algebras}

In the case of the exceptional algebras, the relevant decomposition into subalgebras is:

$$
\begin{aligned}
\mathfrak{e}_{8} & \supset \mathfrak{e}_{7} \times \mathfrak{s u}(2) \\
\mathfrak{e}_{7} & \supset \mathfrak{s o}(12) \times \mathfrak{s u}(2) \\
\mathfrak{e}_{6} & \supset \mathfrak{s u}(6) \times \mathfrak{s u}(2) \\
\mathfrak{f}_{4} & \supset \mathfrak{s p}(6) \times \mathfrak{s u}(2) \\
\mathfrak{g}_{2} & \supset \mathfrak{s u}(2) \times \mathfrak{s u}(2),
\end{aligned}
$$


where the T-brane is embedded in the $\mathfrak{s u}(2)$ subalgebra. The F-theory realization of these $\varepsilon$-deformed T-brane configurations is:

$$
\begin{aligned}
& \mathfrak{e}_{8}: y^{2}=x^{3}+u^{5}+\varepsilon x u^{3} \\
& \mathfrak{e}_{7}: y^{2}=x^{3}+x u^{3}+\varepsilon x^{2} u \\
& \mathfrak{e}_{6}: y^{2}=x^{3}+u^{4}+\varepsilon x u^{2} \\
& \mathfrak{f}_{4}: y^{2}=x^{3}+q u^{4}+\varepsilon x u^{2} \\
& \mathfrak{g}_{2}: y^{2}=x^{3}+q x u^{2}+\varepsilon u^{2} .
\end{aligned}
$$

The factors of $q$ in the non-simply laced cases are introduced in order to pass to the non-split type of each elliptic fiber [42].

\section{T-branes gone wild}

In this section we consider a more general class of T-brane configurations which originate from allowing $\Phi$ to develop higher order poles. More precisely, we now ask whether we can realize a Higgs field of the form:

$$
\Phi=d u\left(\frac{T_{k}}{u^{k}}+\ldots+\frac{T_{1}}{u}+\ldots\right)
$$

where the rightmost set of "..." refers to regular terms in the Higgs field. Here, the generators $T_{k}$ take values in $\mathfrak{g}_{\mathbb{C}}$, the complexification of the gauge algebra for the Hitchin system.

Our plan will be to geometrically engineer such configurations via colliding 7-branes. In particular, we will argue that just as in the case of simple poles, these higher order residues can be understood as background values of matter fields. The main distinction compared with the case of simple poles is that now we allow matter to be localized at a non-reduced scheme $u^{k}=0$. Another goal will be to understand how constraints from anomaly cancellation (in the case of $6 \mathrm{D}$ F-theory vacua) or the condition that a global model exists (in the case of $4 \mathrm{D}$ type IIA vacua) leads to a non-trivial upper bound on the singular behavior possible in such configurations.

The moduli space of solutions for the Hitchin system with wild ramification (i.e. an irregularity singularity) is quite subtle, and is the subject of much work in the mathematics and physical mathematics literature, and originated with the work of Boalch (for a general survey see e.g. [43] and references therein). In appendix A we present a brief overview of some of these results in the case of SU(2) gauge theory with poles of order up to four.

To briefly illustrate some of these subtleties, consider the parameterization of the Hitchin system in terms of the complexified connection $\mathcal{A}=A+\Phi+\Phi^{\dagger}$. The complexified connection will also have poles at the same locations as $\Phi$. The first issue is that although the holonomy of $\mathcal{A}$ detects first order poles (via a residue theorem), higher order poles are not purely topological in form, but appear to depend on a choice of coordinate system near the marked point. Indeed, observe that a complexified gauge transformation:

$$
(d+\mathcal{A}) \mapsto g_{\mathbb{C}}^{-1} \cdot(d+\mathcal{A}) \cdot g_{\mathbb{C}},
$$


can shift the order of higher order poles provided we allow $g_{\mathbb{C}}$ to also be singular at $u$. To deal with such issues, we need to have a more precise notion of which types of singular behavior one should allow.

In physical applications, we can fix some of these ambiguities by requiring that all localized matter fields in the associated geometry remain normalizable. To illustrate, consider a $4 \mathrm{D}$ F-theory vacuum containing a 7-brane gauge theory wrapped on the Kähler surface $C \times T^{2}$. In this system, we can have matter fields localized on either the factor $C$ or the factor $T^{2}$. Consider, then, a matter field which is localized at a point of $T^{2}$, but which transforms as a holomorphic section of a bundle defined on $C$. Following the discussion presented in [44], such matter fields obey an equation of the schematic form:

$$
\left(\frac{\partial}{\partial u}+\mathcal{A}_{u}\right) \cdot \Psi=0
$$

where we assume $\Psi$ transforms in a representation $R$ of the gauge group. The presence of the singularity at $u=0$ means that we must exercise care in writing the normalizable solutions to this equation. For example, we can formally solve equation (4.3) to find:

$$
\Psi^{(i)} \sim \exp \left(\frac{a_{k}^{(i)}}{u^{k-1}}+\ldots\right)
$$

for some $a_{k}^{(i)}$. Here, the superscript $(i)$ labels one component in the vector defined by $\Psi$ in the representation $R$.

The solution (4.4) is only normalizable in the sector of the (complex) $u$-plane where $\operatorname{Re}\left(a_{k}^{(i)} / u^{k-1}\right)<0$. When we pass to another sector, we must take a linear combination of the solutions in this sector to obtain another solution. Following Boalch's work, there are precisely $2(k-1)$ such sectors, i.e. Stokes chambers, and for each one we get a transition matrix from chamber $i$ to chamber $i+1$, which we denote by $S_{i}$. The moduli space problem of interest will then involve holding fixed the generalized monodromy:

$$
\widehat{M}=\exp \left(2 \pi i T_{1}\right) \cdot S_{1} S_{2} \ldots S_{2(k-1)} .
$$

We refer to deformations which hold fixed this data as isomonodromic. Note that the $T_{k}$ of equation (4.1) are still free to vary. For additional details on the theory of isomonodromic deformations of meromorphic differential equations, see for example [45].

Our plan in this section will be to show how to engineer wild T-branes in F-theory. Our main result is that these higher order poles are generated by matter fields localized at non-reduced schemes such as $u^{k}=0$. In this sense, it simply requires additional tuning in the complex structure moduli of an F-theory compactification to realize these more subtle configurations. Now, precisely because this deformation problem is captured by the moduli space of a local Calabi-Yau, constraints from anomaly cancellation bound the number of such matter fields. Moreover, further constraints arise if we attempt to embed the local model in a globally complete geometry. All told, this greatly limits the possible configurations of wild T-branes, including the total order of poles, as well as the possible values of the generalized residues $T_{i}$ which can actually be engineered. To illustrate, we calculate both the physical moduli space (as defined by F-theory) as well as that defined by the wild Hitchin system of isomonodromic deformations. 


\subsection{Wild matter}

We shall now turn to the way in which higher order poles in the Higgs field can arise. To this end, let us consider in more detail the way in which we generate localized modes from the perspective of an 8D 7-brane gauge theory. Along these lines, it is again helpful to work in terms of a 7-brane wrapping a Kähler surface $S=C \times T^{2}$, i.e., we compactify our $6 \mathrm{D}$ theory on an additional $T^{2}$ to four dimensions. Our plan will be to study localized matter fields obtained by Higgsing a parent gauge theory defined on a patch of this Kähler surface. We use a local coordinate $u$ for $C$ and $v$ for the $T^{2}$ factor. Much as in earlier work on modelling intersecting 7-branes using this $8 \mathrm{D}$ gauge theory, matter fields will arise from localized vortex equations. The key difference from earlier work will be in the profile for the parent gauge theory Higgs field we use to trap matter along a non-reduced scheme.

Considering a 7-brane wrapped on a Kähler surface, we can parameterize the higher Kaluza-Klein modes of the system in terms of a collection of $4 \mathrm{D} \mathcal{N}=1$ superfields [24] (see also [46-48]). The resulting supersymmetric equations of motion for the system dictate the profiles of the internal fields. To capture the main features of higher order poles in the Higgs field, it is enough to track the F-term equations of motion, modulo complexified gauge transformations. That is, we shall exclusively work in holomorphic gauge. This will make the match with complex geometry especially transparent, and with no loss of generality. ${ }^{7}$ For a 7 -brane with no localized matter, the F-term equations of motion are governed by the superpotential $[3,24,49,50]$ :

$$
W_{\text {bulk }}=\int_{S} \operatorname{Tr}\left(\Phi_{(2,0)} \wedge F_{(0,2)}\right) .
$$

The first order equations of motion for this system are:

$$
\bar{\partial}_{A} \Phi=0 \quad \text { and } \quad F_{(0,2)}=0 .
$$

Next, expand around a specific background $A^{(0)}$ and $\Phi^{(0)}$ which satisfies these equations of motion, allowing $\Phi^{(0)}$ to possibly have poles along a divisor of $S$. Expanding around this background, we write:

$$
\begin{aligned}
& A=A^{(0)}+A^{(1)}, \\
& \Phi=\Phi^{(0)}+\Phi^{(1)} .
\end{aligned}
$$

Plugging this into the original system of equations, we obtain the first order F-term relations:

$$
\bar{\partial}_{A^{(0)}} \Phi^{(1)}+\left[A^{(1)}, \Phi^{(0)}\right]=0 \quad \text { and } \quad \bar{\partial}_{A^{(0)}} A^{(1)}=0 .
$$

Following [49], since $\left(\bar{\partial}_{A^{(0)}}\right)^{2}=0$ we can express our solution in a local gauge as:

$$
\Phi^{(1)}=\left[\xi, \Phi^{(0)}\right]+h \quad \text { and } \quad A^{(1)}=\bar{\partial}_{A^{(0)}} \xi,
$$

\footnotetext{
${ }^{7}$ The passage back to a unitary frame where we impose F- and D-terms modulo unitary gauge transformations is achieved by a suitable complexified gauge transformation (see e.g. [3, 49]).
} 
for $h$ a holomorphic $(2,0)$ form valued in $\operatorname{ad} P$ with $P$ a principal $G_{\text {parent-bundle, and } \xi \text { a }}$ $(0,0)$ form valued in $\operatorname{ad} P$.

To proceed further, we now assume a specific form for $\Phi^{(0)}$. In terms of the local coordinates $u$ and $v$ introduced previously, $\Phi^{(0)}$ takes the form:

$$
\Phi^{(0)}=\phi d u \wedge d v
$$

for $\phi$ an adjoint valued scalar in the complexification of $\mathfrak{g}_{\mathbb{C}}^{\text {parent }}$. Denote the adjoint action by $\phi$ as $\operatorname{ad}_{\phi}$. In this case, we can make a further decomposition of the adjoint action according to the decomposition into irreducible representations of the unbroken gauge group. Assuming that we have a parent gauge group $G_{\text {parent }}$ which breaks to $G$ (which may contain multiple semi-simple factors), we have a further decomposition into irreducible representations of the original adjoint representation:

$$
\operatorname{ad}\left(G_{\text {parent }}\right)=\underset{i}{\oplus} R_{i}
$$

For each such irreducible representation, denote the eigenvalue of $\operatorname{ad}_{\phi}$ by $\alpha_{R}$. The resulting matter fields transforming in a representation $R$ of $G$ then satisfy the equations:

$$
\Phi_{R}^{(1)}=\alpha_{R} \xi_{R}+h_{R} \quad \text { and } \quad A_{R}^{(1)}=\bar{\partial}_{A^{(0)}} \xi_{R},
$$

in the obvious notation. We can then present localized solutions as:

$$
\Phi_{R}^{(1)}=\alpha_{R} \xi_{R}+h_{R} \quad \text { and } \quad A_{R}^{(1)}=\bar{\partial}_{A^{(0)}}\left(\frac{\Phi_{R}^{(1)}-h_{R}}{\alpha_{R}}\right) .
$$

We obtain a class of solutions by taking $\phi$ valued in the Cartan subalgebra with simple zeros. For example, we can consider the breaking pattern induced by taking:

$$
\phi=\left[\begin{array}{ll}
M u 1_{N \times N} & \\
& -N u 1_{M \times M}
\end{array}\right] .
$$

In this case, we have localized modes in the bifundamental representation of $\operatorname{SU}(N) \times$ $\mathrm{SU}(M)$, which are trapped at $u=0$ :

$$
\Phi_{N \times M}^{(1)}=(M+N) u \xi_{N \times M}+h_{N \times M} \quad \text { and } \quad A_{N \times M}^{(1)}=\bar{\partial}_{A^{(0)}}\left(\frac{\Phi_{N \times M}^{(1)}-h_{N \times M}}{u}\right),
$$

where the subscript $R$ denotes the representation with respect to the gauge group left unbroken by the background choice of $\phi$.

We can also entertain more general polynomials in $u$ :

$$
\phi=\left[\begin{array}{ll}
M \alpha_{R}(u) 1_{N \times N} & \\
& -N \alpha_{R}(u) 1_{M \times M}
\end{array}\right],
$$

which yields the zero modes:

$$
\Phi_{N \times M}^{(1)}=(M+N) \alpha_{R}(u) \xi_{N \times M}+h_{N \times M} \quad \text { and } \quad A_{N \times M}^{(1)}=\bar{\partial}_{A^{(0)}}\left(\frac{\Phi_{N \times M}^{(1)}-h_{N \times M}}{\alpha_{R}(u)}\right) .
$$


Provided $\alpha_{R}(u)$ has simple zeros, we get localized matter in the bifundamental of $\mathrm{SU}(N) \times$ $\mathrm{SU}(M)$. If, however, multiple zeroes coincide, we instead obtain a higher order pole.

Returning to the general thread of our discussion, we see that the localized modes which descend from bulk modes are captured in terms of the quantity $\xi$, which has the local expression:

$$
\xi_{R}=\frac{\psi(u)}{\alpha_{R}(u)}, \text { and } \xi_{R^{c}}=\frac{\psi^{c}(u)}{\alpha_{R}(u)} .
$$

Here, we have used the fact that a full hypermultiplet localizes together (see appendix B of reference [3]). The general statement, then, is that for hypermultiplet matter $\psi \oplus \psi^{c}$ localized at the zeroes of $\alpha_{R}(u)$, we have local representatives in:

$$
\psi \in K_{T^{2}}^{1 / 2} \otimes R \otimes \frac{\mathbb{C}[u]}{\left(\alpha_{R}(u)\right)} \quad \text { and } \quad \psi^{c} \in K_{T^{2}}^{1 / 2} \otimes R^{c} \otimes \frac{\mathbb{C}[u]}{\left(\alpha_{R}(u)\right)},
$$

that is, we can write down power series expansions:

$$
\psi(u)=\sum_{i=0}^{k-1} \psi_{i} u^{i} \quad \text { and } \quad \psi^{c}(u)=\sum_{i=0}^{k-1} \psi_{i}^{c} u^{i} .
$$

In the above expressions, we note in passing that both $\psi$ and $\psi^{c}$ also transform as spinors on the matter curve $T^{2}$ factor, i.e. we have included a factor of $K_{T^{2}}^{1 / 2}$.

Consider now the coupling of these localized modes to the other bulk degrees of freedom of the system. For a local model with matter generated by $\alpha_{R}=u^{k}$, we get $k$ zero modes all localized at $u=0$. Therefore, plugging into our bulk superpotential, we can read off the coupling of the bulk gauge field to these boundary modes:

$$
W_{T^{2}}=\int_{S} \bar{\partial}_{\bar{u}}\left(\Phi_{R^{c}}^{(1)} \cdot\left(\bar{\partial}_{\bar{v}}+A_{\bar{v}}\right) \cdot A_{R}^{(1)}-\Phi_{R}^{(1)} \cdot\left(\bar{\partial}_{\bar{v}}+A_{\bar{v}}\right) \cdot A_{R^{c}}^{(1)}\right),
$$

where $u$ is a local coordinate transverse to the matter curve and $v$ is a local coordinate along the matter curve. In this expression, we have also kept implicit the pairing with respect to just one of the simple gauge group factors, namely the one localized on $S=C \times T^{2}$. We trace over the representation content of the other gauge group factors. As an example of this procedure, consider the case of $G=\mathrm{SU}(N)$, with each matter field a bifundamental of $\mathrm{SU}(N) \times \mathrm{SU}(M)$. In this case, we trace over the flavor index, i.e. the index of $\mathrm{SU}(M)$, whilst allowing a non-trivial covariant derivative of $\mathrm{SU}(N)$ to act on the localized matter fields.

In arriving at (4.23) we have used the fact that there is a natural symplectic pairing between the scalars of the hypermultiplet [3]. Inserting our expressions for the localized fluctuations $\Phi_{R}^{(1)}$ and $A_{R}^{(1)}$ from (4.14) and (4.20) into $W_{T^{2}}$ then yields:

$$
W_{T^{2}}=\int_{S} \bar{\partial}_{\bar{u}}\left(\frac{\psi^{c}(u) \cdot\left(\bar{\partial}_{\bar{v}}+A_{\bar{v}}\right) \cdot \psi(u)}{u^{k}}\right) .
$$

Note that in the above, we have a power series expansion in $u$ for $\psi(u)$ and $\psi^{c}(u)$. So, although the product of $\psi(u)$ and $\psi^{c}(u)$ has terms of degree zero to degree $2(k-1)$, the 
only terms which actually survive are from degree zero to degree $k-1$, the higher order terms being regular, and thus annihilated by $\bar{\partial}_{\bar{u}}$. Another feature of this formula is that there is a non-degenerate pairing of the simple poles. Such terms can be evaluated via a residue integral, and yield standard kinetic terms on the $T^{2}$ factor $[3,49]$.

The higher order poles present in (4.24) are what generate wild behavior in our Hitchin system. To see this, consider the equations of motion obtained by varying with respect to the bulk fields of the system. The bulk F-term equations of motion are now:

$$
\bar{\partial}_{A} \Phi=\bar{\partial}_{\bar{u}}\left(\frac{\left\langle\left\langle\psi^{c}(u), \psi(u)\right\rangle\right\rangle}{u^{k}}\right) \quad \text { and } \quad F_{(0,2)}=0
$$

A similar expression holds for the D-term equations of motion. Much as in [24], we have introduced a canonical pairing $\left\langle\left\langle\psi^{c}(u), \psi(u)\right\rangle\right\rangle$ valued in $K_{T^{2}} \otimes \operatorname{ad} P$, with $P$ a principal $G$ bundle. Here, we also trace over the flavor indices. For example, in the special case of $G=\mathrm{SU}(N)$ and a flavor group $\mathrm{SU}(M)$, we write, for $\alpha$ an index for the adjoint representation of $G$ :

$$
\left\langle\left\langle\psi^{c}(u), \psi(u)\right\rangle\right\rangle_{\alpha}=\sum_{m} \psi_{m}^{c}(u) \cdot V_{\alpha}^{(R)} \cdot \psi_{m}(u),
$$

where $V_{\alpha}^{(R)}$ is a generator of the algebra in the representation $R$.

Let us now collect the terms of the outer product $\left\langle\left\langle\psi(u), \psi^{c}(u)\right\rangle\right\rangle$ in terms of a collection of $k$ rank $M$ matrices, $T_{1}, \ldots, T_{k}$. Strictly speaking, we view the $T_{j}$ as holomorphic sections of $K_{T^{2}}$ valued in the adjoint representation of $G$. Since, however, $K_{T^{2}}$ is trivial, we can freely switch between these conventions. Explicitly, the terms of the outer product are obtained by expanding our power series and keeping all terms which are not regular in our meromorphic expansion. Doing so, we arrive at the final form of the F-term equations of motion:

$$
\bar{\partial}_{A} \Phi=\bar{\partial}_{\bar{u}}\left(\frac{T_{k}+\ldots+T_{1} u^{k-1}}{u^{k}}\right) \quad \text { and } \quad F_{(0,2)}=0 .
$$

One can also express the right hand side as:

$$
\bar{\partial}_{A} \Phi=2 \pi i\left(\delta_{u} T_{1}+\sum_{j=1}^{k-1} \frac{(-1)^{j}}{j !} \partial_{u}^{j} \delta_{u} T_{j+1}\right) .
$$

Locally, then, we have:

$$
\Phi \sim d u\left(\frac{T_{k}}{u^{k}}+\ldots+\frac{T_{1}}{u}+\text { regular terms at }(u=0)\right),
$$

that is, we obtain the expected behavior of the Higgs field. Of course, we are now free to restrict to the case of the Hitchin system, i.e., by decompactifying the $T^{2}$ factor.

Another approach to the study of Hitchin systems with poles is the use of star shaped quivers (see e.g. [51-53] and associated work on hyperpolygons [54]). Mathematically, the connections between such quivers and wild Hitchin systems have been viewed as a novel correspondence between disparate geometric objects. Here, we would like to understand how this structure emerges naturally from a physical point of view. Since we have an $\mathrm{SU}(N)$ 
gauge theory, we have a central quiver node with this gauge group. In mathematical terms, we have a copy of the fundamental representation, namely the vector space $\mathbb{C}^{N}$. Now, once we include the presence of intersections with additional 7 -branes, there are additional bifundamental fields, the $\psi \oplus \psi^{c}$. For each stack of $M_{i}$ 7-branes intersecting the Hitchin curve at a marked point $p_{i}$, we have a $\mathrm{SU}\left(M_{i}\right)$ flavor symmetry, with defining representation a copy of $\mathbb{C}^{M_{i}}$. As we have already remarked, the order of vanishing for the parent Higgs field dictates the total number of such bifundamentals, so we introduce an additional label as $\psi_{(1, i)} \oplus \psi_{(1, i)}^{c}, \ldots, \psi_{\left(k_{i}, i\right)} \oplus \psi_{\left(k_{i}, i\right)}^{c}$. For each such pair, we get maps:

$$
\begin{aligned}
& \psi_{(s, i)} \in \operatorname{Hom}\left(\mathbb{C}^{N}, \mathbb{C}^{M_{i}}\right), \\
& \psi_{(s, i)}^{c} \in \operatorname{Hom}\left(\mathbb{C}^{M_{i}}, \mathbb{C}^{N}\right) .
\end{aligned}
$$

See figure 2 for a depiction of this quiver. We construct a higher order pole for the Higgs field of the Hitchin system using suitable bilinears in the $\psi$ and $\psi^{c}$ 's. Referring to this pairing as before, namely $\langle\langle\cdot, \cdot\rangle\rangle$, we have:

$$
\Phi \sim d u \sum_{i} \sum_{l_{i}=1}^{k_{i}} \frac{\sum_{s+t=l_{i}}\left\langle\left\langle\psi_{(s, i)}^{c}, \psi_{(t, i)}\right\rangle\right\rangle}{\left(u-p_{i}\right)^{l_{i}}}+\ldots
$$

where the ellipsis "..." refers to regular terms. In this case, the notion of Stokes chambers and Stokes data must be consistently combined across different patches. We refer to appendix A for some examples of this analysis.

\subsubsection{Coordinate free formulation}

In our physical derivation of wild Higgs fields, we made use of a particular coordinate system with matter localized at the non-reduced scheme $u^{k}=0$. Indeed, as we have already remarked, one of the subtle features of wild Higgs fields is the fact that the higher order poles are not detected by a residue formula. In this subsection we develop a coordinate free formulation of the same data obtained above.

Consider the curve $C$ of genus $g$, with marked points $p_{i}$ with multiplicities $n_{i}$. We view the $p_{i}$ as the locations of the poles for the Higgs field, and the $n_{i}$ as the order of each pole. Given the effective divisor $D=\sum_{i} n_{i} p_{i}$, we consider the sheaf $\mathcal{O}_{D}$ of holomorphic functions on $D$ as a subscheme of $C$, so that multiplicities are considered. Since functions can be multiplied, $\mathcal{O}_{D}$ is a sheaf of rings. Then the ring $\mathbb{C}[u] /\left(\alpha_{R}(u)\right)$ appearing in $(4.21)$ can be more intrinsically written as $H^{0}\left(\mathcal{O}_{D}\right)$. Another useful way to think of $\mathcal{O}_{D}$ is as the quotient $\mathcal{O}_{C}$ by the ideal sheaf of functions vanishing on $D$ (including multiplicities). Since the ideal sheaf of $D$ is isomorphic to $\mathcal{O}_{C}(-D)$, we have a short exact sequence

$$
0 \rightarrow \mathcal{O}_{C}(-D) \rightarrow \mathcal{O}_{C} \rightarrow \mathcal{O}_{D} \rightarrow 0
$$

It turns out that there is a natural notion of differentials with poles on $D$ including multiplicity in terms of the notion of the dualizing sheaf $\omega_{D}$ of $D$ [55]. This is a generalization to singular schemes of the canonical bundle of a smooth variety. Since $D$ is just a collection of points, there is a simpler coordinate-free description of $\omega_{D}$ in terms of 1 -forms 


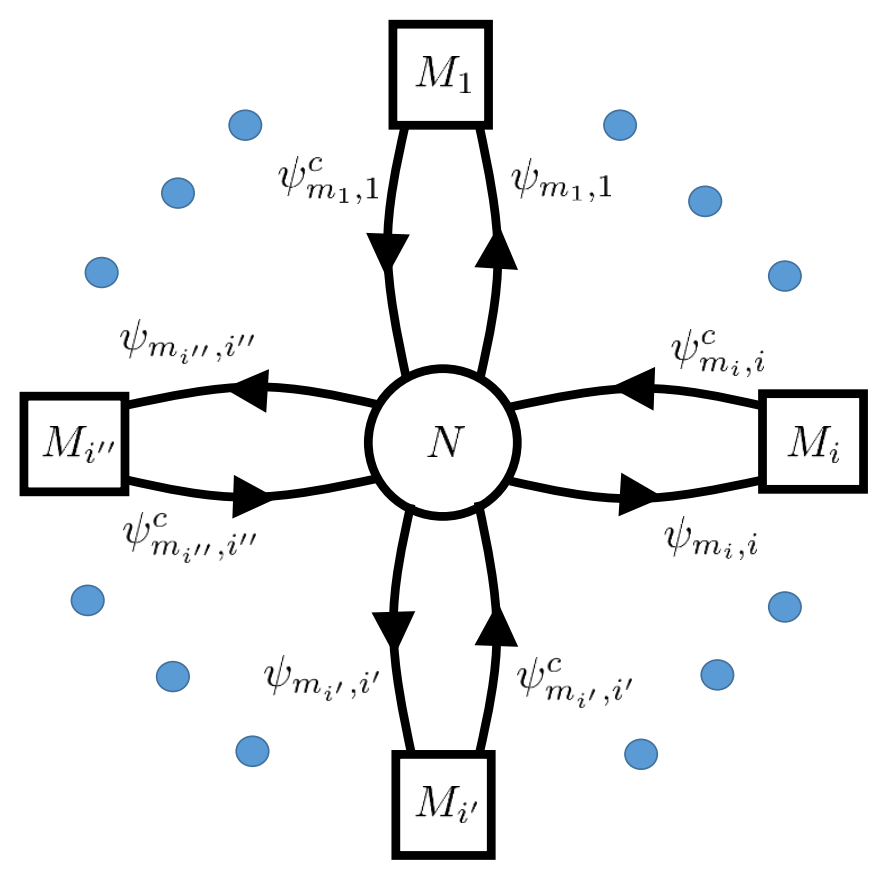

Figure 2. Depiction of the star shaped quiver generated by an intersecting brane configuration in F-theory. The central node corresponds to the contribution from the 7-brane wrapped over the gauge theory curve, and the satellite nodes indicated as squares correspond to the flavor branes of the system. These intersect the Hitchin system curve at points, and for $k$ such intersecting fields, there are regions in the moduli space which are represented by higher poles in the Hitchin system Higgs field. Each such satellite node corresponds to the location of a distinct marked point.

and poles (which we will also derive below), but we include the more general description of the dualizing sheaf here in anticipation of applications to $4 \mathrm{D}$ models in which we can have defects along singular curves.

The dualizing sheaf can be computed as in [55] by considering

$$
\omega_{D}=\underline{\operatorname{Ext}}_{\mathcal{O}_{C}}^{1}\left(\mathcal{O}_{D}, \mathcal{O}\left(K_{C}\right)\right)
$$

where $\omega_{C}=\mathcal{O}\left(K_{C}\right)$ is usual sheaf associated with the canonical bundle of $C$ and $\underline{\text { Ext }}_{\mathcal{O}_{C}}^{1}$ denotes the Ext sheaf, rather than the Ext group. Applying the long exact sequence of $\underline{\operatorname{Ext}}_{\mathcal{O}_{C}}^{*}\left(\cdot, \mathcal{O}\left(K_{C}\right)\right)$ to $(4.34)$ gives

$$
\begin{aligned}
\underline{\operatorname{Ext}}_{\mathcal{O}_{C}}^{0}\left(\mathcal{O}_{C}, \mathcal{O}\left(K_{C}\right)\right) & \rightarrow \operatorname{Ext}_{\mathcal{O}_{C}}^{0}\left(\mathcal{O}_{C}(-D), \mathcal{O}\left(K_{C}\right)\right) \\
& \rightarrow \operatorname{Ext}_{\mathcal{O}_{C}}^{1}\left(\mathcal{O}_{D}, \mathcal{O}\left(K_{C}\right)\right) \rightarrow \underline{\operatorname{Ext}}_{\mathcal{O}_{C}}^{1}\left(\mathcal{O}_{C}, \mathcal{O}\left(K_{C}\right)\right) .
\end{aligned}
$$

The terms in (4.35) can all be identified since $\underline{\operatorname{Ext}}_{\mathcal{O}_{C}}^{0}$ is just the Hom sheaf, while

$$
\underline{\operatorname{Ext}}^{1}\left(\mathcal{O}_{C}, \mathcal{O}\left(K_{C}\right)\right)=0
$$

since $\mathcal{O}_{C}$ is locally free. So (4.35) becomes

$$
\mathcal{O}\left(K_{C}\right) \rightarrow \mathcal{O}\left(K_{C}+D\right) \rightarrow \omega_{D} \rightarrow 0
$$


Moreover, the first map in (4.37) is just multiplication by the local equations defining $D$, and thus we see that $\omega_{D}$ is just the restriction of $\mathcal{O}\left(K_{C}+D\right)$ to $D$ :

$$
\omega_{D}=\left.\mathcal{O}\left(K_{C}+D\right)\right|_{D}=: \mathcal{O}_{D}\left(K_{C}+D\right) .
$$

At a point $p$ with local coordinate $u$ occurring with multiplicity $k$ in $D$, then the sections of $\omega_{D}$ at $p$ are precisely the expressions

$$
d u\left(\frac{T_{k}}{u^{k}}+\ldots+\frac{T_{1}}{u}\right)
$$

modulo regular terms, since we have modded out by the regular differentials $\mathcal{O}\left(K_{C}\right)$ in (4.37).

Now $\omega_{D}$ is a sheaf of modules on $D$ rather than a sheaf of rings. In down to earth terms, though we cannot multiply differentials, we can multiply functions and differentials to get a differential. Furthermore, $\omega_{D}$ is a free sheaf and hence one has an isomorphism of sheaves of modules (not rings):

$$
\beta: \mathcal{O}_{D} \simeq \omega_{D}
$$

In this language the apparent coordinate dependence in the wild system is the statement that $\beta$ is not intrinsic. The ambiguity that we faced earlier in the choice of local coordinates is replaced by an ambiguity in a choice of isomorphism (4.40), which occurs only at the points of $D$ rather than in a neighborhood. We can use coordinates to define an isomorphism by

$$
T_{k}+\ldots T_{1} u^{k-1} \mapsto\left(\frac{T_{k}}{u^{k}}+\ldots+\frac{T_{1}}{u}\right) d u
$$

as an isomorphism which respects multiplication by sections of $\mathcal{O}_{D}$. But any isomorphism of modules will do.

More abstractly, we can see (4.40) without computation by noting that $D$ is locally defined by one equation (e.g. by $\alpha$ in our earlier notation) hence $D$ is Gorenstein, which implies that $\omega_{D}$ is locally free [55]. Then since $D$ just consists of finitely many points, the local isomorphism is in fact a global isomorphism.

From the above analysis, we can rewrite $(4.21) \mathrm{as}^{8}$

$$
\psi \in R \otimes H^{0}\left(\mathcal{O}_{D}\right), \quad \psi^{c} \in R^{c} \otimes H^{0}\left(\mathcal{O}_{D}\right)
$$

Then to get the F-term equation of motion, we combine multiplication in $\mathcal{O}_{\mathcal{D}}$, the representation-theoretic pairing $\langle\langle\cdot, \cdot\rangle\rangle$, and the isomorphism $\beta$ to define $\beta\left(\left\langle\left\langle\psi, \psi^{c}\right\rangle\right\rangle\right)$, an adjointvalued section of $\omega_{D}$, which is just the polar part of a 1 form at each point of $D$ as explained above. So the equation of motion on the curve $C$ can be rewritten as:

$$
\bar{\partial}_{A}(\Phi)=0 \text { on } C-D \text {, singular part of } \Phi=\beta\left(\left\langle\left\langle\psi, \psi^{c}\right\rangle\right\rangle\right) .
$$

We now check that the physics does not depend on the choice of $\beta$. Given a second isomorphism $\beta^{\prime}: \mathcal{O}_{D} \rightarrow \omega_{D}$, we exhibit an explicit redefinition of the fields $\psi, \psi^{c}$ which

\footnotetext{
${ }^{8}$ Here we drop the factors of $K_{T^{2}}^{1 / 2}$ appearing in (4.21) since we are dealing with the vacua of a $6 \mathrm{D}$ theory.
} 
takes the equation of motion for $\beta$ to the equation of motion for $\beta^{\prime}$. Indeed, consider $\mathfrak{A}=\left(\beta^{\prime}\right)^{-1} \circ \beta$, an automorphism of the free module $\mathcal{O}_{D}$. Let $f=\mathfrak{A}(1) \in H^{0}\left(\mathcal{O}_{D}\right)$. Then using the module homomorphism property of $\mathfrak{A}$, we see that $\mathfrak{A}=m_{f}$, the automorphism $m_{f}(g)=f g$ of $\mathcal{O}_{D}$ given as multiplication by $f$.

Since $\mathfrak{A}$ is an automorphism, we have that $f\left(p_{i}\right) \neq 0$ for each $p_{i}$. So we can find a welldefined square root of $f$ which we write as $\sqrt{f} \in H^{0}\left(\mathcal{O}_{D}\right)$. Then via the field redefinition for $\psi$

$$
R \otimes H^{0}\left(\mathcal{O}_{D}\right) \stackrel{1 \otimes m_{\sqrt{f}}}{\longrightarrow} R \otimes H^{0}\left(\mathcal{O}_{D}\right)
$$

and a similar field redefinition for $\psi^{c}$, we verify that the equation of motion for $\beta$ are transformed into the equation of motion for $\beta^{\prime}$, since the combined effect of these transformations on $\left\langle\left\langle\psi, \psi^{c}\right\rangle\right\rangle$ is just $1 \otimes m_{f}$. The treatment of bifundamental matter works in the same fashion, in which case one includes a sum over flavors in our definition of the pairing $\langle\langle\cdot, \cdot\rangle\rangle$.

\subsection{Moduli spaces}

Now that we have presented a general method for constructing Higgs fields with higher order poles, it is natural to ask about the resulting moduli space of vacua.

To a certain extent, this depends on the physical context of the problem. If we treat the Hitchin system with wild ramification as the full system, then we have a well-defined moduli space problem which has previously been studied in the math literature. We shall shortly review this parametrization of the moduli space, but first we would like to understand what sort of constraints embedding in F-theory or type IIA string theory imposes on these systems.

To illustrate some of these points, consider first the case of $6 \mathrm{D}$ F-theory vacua. For a $6 \mathrm{D}$ gauge theory with gauge group $G$, there is typically a coupling to $6 \mathrm{D}$ tensor multiplets as well as $6 \mathrm{D}$ hypermultiplets. Anomaly cancellation for each such gauge group factor imposes tight constraints on the total number of hypermultiplets which are present. For example, in the case of a local base $\mathcal{O}(-2) \rightarrow \mathbb{P}^{1}$, consider an $\mathrm{SU}(N)$ 7-brane wrapped on the $\mathbb{P}^{1}$. Cancellation of anomalies then yields the condition that there are precisely $F=2 N$ matter fields in the fundamental representation of $\mathrm{SU}(N)$. Similar considerations hold for other curves and gauge group factors for $6 \mathrm{D}$ vacua.

Such constraints are seemingly less stringent in the related context of $4 \mathrm{D} \mathcal{N}=2$ vacua obtained, for example, by compactifying on a further $T^{2}$ factor. For example, as this is not a chiral theory in four dimensions, the constraints from gauge anomaly cancellation are no longer present. There are still constraints, however, because at least in a local model, we must require that we can write a complete metric for the local Calabi-Yau in the neighborhood of the Hitchin system curve. Otherwise, we must include additional sectors and / or reintroduce gravity into the model. If gravity is to remain decoupled on a -2 curve engineering an $\mathrm{SU}(N)$ gauge theory, we must require that the number of $4 \mathrm{D}$ hypermultiplets in the fundamental representation is $F \leq 2 N$. So again, we see that physical constraints limit the total number of matter fields.

But once we accept that the total number of matter fields is bounded above, we must also accept that the order of poles in any wild Hitchin system will also be bounded 
above. Indeed, our whole method for generating higher order poles relies on activating background values for these matter fields. To illustrate, we see that for $k_{i}$ hypermultiplets transforming in the bifundamental representation $\left(\mathbf{N}, \overline{\mathbf{M}_{i}}\right)$, the total number of flavors $F_{\mathbf{N}}$ in the fundamental representation is:

$$
F_{\mathbf{N}}=\sum_{i} k_{i} M_{i}
$$

Neglecting the singlet moduli (which we associate with a decoupled gravitational sector), the moduli space swept out by these charged fields are associated with the bulk Higgs field, the gauge connection, modulo complexified gauge transformations, for a total of $(2 g-$ 2) $\operatorname{dim} G$ complex degrees of freedom, as well as $F_{R}$ localized matter fields in some representation $R$ of the gauge group. The dimension of the moduli space from these 7-branes is then:

$$
\operatorname{dim} \mathcal{M}_{7 \text {-branes }}=(2 g-2) \operatorname{dim} G+\sum_{R} 2 F_{R} \times \operatorname{dim} R,
$$

in the obvious notation.

Let us now match this structure to the moduli space of the wild Hitchin system. As we have already remarked, some care must be exercised in even defining this moduli space, since we are dealing with a singular field configuration. For simplicity, consider the moduli space on a genus $g$ curve with a single marked point at $u=0$ in which the Higgs field has singular part:

$$
\Phi=d u\left(\frac{T_{k}}{u^{k}}+\ldots+\frac{T_{1}}{u}\right) .
$$

Given some fixed choice of the $T_{i}$ 's one can consider solutions to the Hitchin system. This question is studied in $[43,44,56,57]$ for the case where all $T_{j}$ are regular and semi-simple (that is, diagonalizable with all eigenvalues non-zero). Call this moduli space $\mathcal{M}_{H}\left(T_{1}, \ldots, T_{k}\right)$. One can also consider the moduli space associated where $T_{1}$ is not held fixed, which we denote by $\mathcal{M}_{H}\left(T_{2}, \ldots, T_{k}\right)$. In reference [44], the former is denoted by $\mathcal{M}_{H}\left(T_{1}\right)$ while the latter is denoted by $\mathcal{M}_{H}$. This notation emphasizes the point that the complex structure and symplectic structure of the moduli space does not depend on the higher order polar terms. To avoid confusion, we shall keep manifest all $k$ terms in what follows.

The dimension of the moduli space is calculated in [44, 57], with the end result:

$$
\operatorname{dim} \mathcal{M}_{H}\left(T_{1}, \ldots, T_{k}\right)=(2 g-2) \operatorname{dim} G+k(\operatorname{dim} G-\operatorname{rk} G) .
$$

The contribution $(2 g-2) \operatorname{dim} G$ is the dimension of the Hitchin moduli space on a genus $g$ curve, and the additional terms are associated with a single order $k$ pole. From the parameters $T_{1}, \ldots, T_{k}$, we have $k \times \operatorname{dim} G$ complex parameters. Of these, a topological interpretation either from a holonomy or topological Stokes data can be given to $k \times$ $\operatorname{dim} G-(k-1) \times \operatorname{rk} G$ of these parameters. The remaining $(k-1) \times \operatorname{rk} G$ parameters are then associated with isomonodromic deformations [45]. See appendix A for further discussion as well as some explicit examples. 
Let us now determine the dimension of the moduli space in accord with an algebrogeometric construction, geared towards an eventual F-theory construction. We calculate the contribution to the various Casimir invariants which are independent of regular terms. Focusing on the special case $G=\mathrm{SU}(N)$, we have:

$$
\operatorname{Tr}\left(\Phi^{j}\right)=d u^{\otimes j}\left(\frac{\operatorname{Tr}\left(T_{k}^{j}\right)}{u^{j k}}+\ldots+\frac{j \operatorname{Tr}\left(T_{k}^{j-1} T_{1}+\ldots\right)}{u^{k(j-1)+1}}+(\text { contributions from regular terms })\right) .
$$

Following [44], expressions of the form (4.49) form an affine space isomorphic to

$$
H^{0}\left(C, K_{C}^{j} \otimes \mathcal{O}(p)^{k(j-1)}\right),
$$

since any two expressions of the form (4.49) differ by a $j$-differential with a pole at $p$ of order at most $k(j-1)$.

Let us count the dimension of this moduli space. From Riemann-Roch, we have:

$$
\operatorname{dim} H^{0}\left(C, K_{C}^{j} \otimes \mathcal{O}(p)^{k(j-1)}\right)=\left(j-\frac{1}{2}\right)(2 g-2)+k(j-1) .
$$

Summing over the independent Casimirs, i.e. from $j=2$ to $j=N$, we have the total number of such deformation moduli is:

$$
\operatorname{dim} \mathcal{M}_{\text {cplx }}=\sum_{j=2}^{N} \operatorname{dim} H^{0}\left(C, K_{C}^{j} \otimes \mathcal{O}(p)^{k(j-1)}\right)=\frac{1}{2}[(2 g-2) \operatorname{dim} G+k(\operatorname{dim} G-\operatorname{rk} G)],
$$

where in the above we used the fact that $\operatorname{dim} G=N^{2}-1$ and $\operatorname{rk} G=N-1$. Now, in addition to the complex structure moduli captured by the base of the Hitchin system, we also have (in the IIA formulation) the intermediate Jacobian of the Calabi-Yau threefold defined by the local model. In the Hitchin system formulation, these originate from the Prym variety of the spectral curve $[21,58]$. The total dimension of the moduli space is therefore:

$$
\operatorname{dim} \mathcal{M}_{H}\left(T_{1}, \ldots, T_{k}\right)=(2 g-2) \operatorname{dim} G+k(\operatorname{dim} G-\operatorname{rk} G) .
$$

In these calculations, we have used the fact that the relevant moduli can be counted solely from the Casimirs of the Higgs field without regard to the Higgs field itself. This follows from the fibration structure of the integrable system reviewed in appendix B.

\section{Geometric unification}

Our discussion in the previous sections focused on the physical origin of wild Hitchin systems in local F-theory models. To complete the circle of ideas we now present some illustrative examples generated by successive tuning in the limiting behavior of complex structure moduli. We demonstrate that this global perspective also unifies different wild Hitchin systems in one geometric framework.

In a global F-theory model the complex structure moduli can either be localized on a component of the discriminant locus — as in the case of charged matter — or can be 
moduli which transform as singlets under all gauge group factors. In the latter case, such moduli can often be thought of as remnants from Higgsing a higher rank gauge group. This often occurs in the unfolding of colliding singularities. For example, if we have matter fields in a representation $R_{\text {parent }}$ of a parent gauge group $G_{\text {parent }}$, decomposition into irreducible representations of a possibly semi-simple descendant gauge group $G$ can include singlets of any or all of the simple gauge group factors of $G$.

There are also "purely gravitational" gauge singlet hypermultiplets which should best be viewed as moving the locations of various marked points. Geometrically, these define torsion deformations of the local model. In appendix $\mathrm{C}$ we extend the analysis presented in [4], showing that all such deformations are physical moduli in an F-theory model.

Including such closed string sectors provides a unified perspective for wild systems. For example, since we can move the locations of various poles, we can address what happens when different marked points with possibly different matter content and pole orders are brought together at a single location in the geometry. This question is difficult to answer in the wild Higgs bundle literature, because even having wild ramification at different points requires introducing additional gluing conditions known as tentacles which match the Stokes chambers present near distinct marked points. Additionally, there is always the possibility that additional moduli must be incorporated into the structure of the wild system to properly account for such gluing operations. From the geometric perspective, however, all such moduli spaces are on an equal footing and simply correspond to different parameterizations specified by the singlet moduli of the global F-theory model.

To illustrate, consider an $\mathrm{SU}(2)$ 7-brane gauge theory localized on a $\mathbb{P}^{1}$ with selfintersection -2 in an F-theory base. As we have already remarked, 6D anomaly cancellation requires precisely four matter fields in the fundamental representation of $\mathrm{SU}(2)$. These matter fields may be localized at distinct points of the curve, or could be collected together at the same point. As we do this, the pole structure, as well as flavor 7-branes of the system will also change. The moduli controlling these deformations are those responsible for moving the locations of marked points. As we establish in appendix C, such moduli are part of the physical moduli of an F-theory model.

To provide some further examples, in this section we will focus on F-theory compactified on an elliptically fibered Calabi-Yau threefold $X \rightarrow \mathbb{F}_{n}$ with base a Hirzebruch surface. Consistency of the model requires $-12 \leq n \leq 12$. The minimal Weierstrass model for such geometries is:

$$
y^{2}=x^{3}+f x+g
$$

with:

$$
\begin{aligned}
f(w, u) & \sim \sum_{i} w^{i} f_{8+n(4-i)}(u) \\
g(w, u) & \sim \sum_{j} w^{j} g_{12+n(6-j)}(u)
\end{aligned}
$$

where $i$ is bounded by the largest value less than or equal to 8 such that $8+n(4-I) \geq 0$ and $j$ is bounded by the largest number less than or equal to 12 with $12+n(6-J) \geq 0$. 
Here $w=0$ determines the zero section of the Hirzebruch surface (i.e. the base $\mathbb{P}^{1}$ ) and $u$ is a local coordinate on the base $\mathbb{P}^{1}$. By tuning the Weierstrass coefficients, namely by adjusting the values of charged and gauge singlet hypermultiplets, we will show how to interpolate between various types of wild Hitchin systems.

Our plan in the rest of this section will be to present some illustrative examples, focusing on models where we preserve an $\mathrm{SU}(2)$ gauge group factor. After this, we revisit the analysis presented in [4] for the F-theory dual to the Heterotic tangent bundle on a $K 3$ surface.

\subsection{A wild compact SU(2) model}

To illustrate the above points, let us consider an explicit example. We consider an F-theory model with base $\mathbb{F}_{n}$ for $n$ a non-negative integer. We will be interested in the case where the $w=0$ locus supports an $\mathfrak{s u}(4)$ gauge symmetry, which is associated with an $I_{4}$ fiber. Recall that this means $f$ and $g$ must not vanish along $w=0$ but the discriminant must vanish to order $w^{4}$. Furthermore, we will require that this locus intersects a curve supporting an $\mathfrak{s u}(2)$ symmetry (i.e. $I_{2}$ fiber). Producing a product group requires tuning singularities on two loci in the base, $w=0$ and $w=\epsilon$ simultaneously, which is difficult to identify when $f$ and $g$ are expanded in $w$ alone. This tuning process was described in [59], where $f$ and $g$ can be systematically expanded in $w(w-\epsilon)$. Letting $\sigma=w-\epsilon$ for brevity:

$$
\begin{aligned}
& f=F_{0}+F_{1} w \sigma+F_{2} w^{2} \sigma^{2}+\ldots \\
& g=G_{0}+G_{1} w \sigma+G_{1} w^{2} \sigma^{2}+\ldots,
\end{aligned}
$$

where $F_{i}=f_{2 i}+f_{2 i+1} u$ and $G_{i}=g_{2 i}+g_{2 i+1} w$. For this example, we will set $\epsilon=\epsilon_{1} \beta$ (note that in the limit $\epsilon_{1} \rightarrow 0$ this leads to an $\mathrm{SU}(6)$ theory).

It is convenient to define the $\mathfrak{s u}(2)$ locus as $\sigma=w-\beta \epsilon_{1}$ where $\beta$ is a polynomial of degree $r$ and $\epsilon_{1}$ has degree $n-r$ over the $\mathbb{P}^{1}$ base. To leading order the Weierstrass coefficients take the form

$$
\begin{aligned}
& f=-\frac{\alpha^{4} \beta^{4}}{48}+\frac{1}{18}\left(2 \alpha^{2} \beta^{2} \epsilon_{1} \phi-3 \alpha^{2} \beta^{3} \nu\right) w+\ldots \\
& g=\frac{\alpha^{6} \beta^{6}}{864}+\frac{1}{216}\left(3 \alpha^{4} \beta^{5} \nu-2 \alpha^{4} \beta^{4} \epsilon_{1} \phi\right) w+\ldots
\end{aligned}
$$

with corresponding discriminant locus

$$
\Delta=(\sigma)^{2}\left(w^{4}\right)\left(\frac{1}{5184}\left(\alpha^{4} \beta^{2}\right)\left[12 \beta \phi^{3}\left(\alpha^{2}+2 \nu \epsilon_{1}\right)+\ldots\right]+\mathcal{O}(w)+\ldots\right)
$$

where in addition to the functions $\beta$ and $\epsilon_{1}$, the solution is parameterized by functions $\alpha$ (of degree $2+n-r$ ), $\phi$ (of degree $4+r$ ), and $\nu$ (of degree $4+n-r$ ).

The total matter content ${ }^{9}$ of the theory is determined by the two integers $(n$ and $r$ ) and is given in table 1 . It is clear that $\epsilon_{1}=0$ counts the bifundamental matter, while

\footnotetext{
${ }^{9}$ The singlet moduli counted above are only those contributing on the patch containing $w=0$. The degrees of freedom in $f_{i}$ with $i \geq 4$ and $g_{j}$ with $j \geq 6$ are unconstrained and omitted from the count above. In the heterotic dual theory these degrees of freedom are merely the 20 moduli of the heterotic $K 3$ and the $-30 n+112$ moduli associated to an $E_{8}$ bundle with $c_{2}=12-n$.
} 


\begin{tabular}{|cccc|}
\hline Representation & Multiplicity & Representation & Multiplicity \\
\hline$(\mathbf{1}, \mathbf{1})$ & $4 n-2 r+22$ & $\mathbf{( 4 , \mathbf { 1 } )}$ & $2 n+2 r+16$ \\
$(\mathbf{6}, \mathbf{2})$ & $\frac{r}{2}$ & $\mathbf{( 4 , 2 )}$ & $n-r$ \\
$(\mathbf{1}, \mathbf{2})$ & $2 n+r+16$ & $\mathbf{( 6 , 1 )}$ & $n-r+2$ \\
\hline
\end{tabular}

Table 1. The multiplicity of matter fields (in full hypermultiplets) in the $\mathrm{SU}(4) \times \mathrm{SU}(2)$ theory on base $\mathbb{F}_{n}$.

$\alpha=0$ corresponds to the $(\mathbf{6}, \mathbf{1})$. Intersections with the $I_{1}$ component of the discriminant gives $2 n+16+2 r(\mathbf{4}, \mathbf{1})$ multiplets and $2 n+16+r(\mathbf{2}, \mathbf{1})$ multiplets. The locus with $\beta=0$ corresponds to a $D_{5}$ enhancement which gives $\frac{r}{2}(\mathbf{6}, \mathbf{2})$ multiplets. Observe that especially for the $\mathrm{SU}(4)$ gauge theory, we have matter fields in different representations. This can also be covered in a local model, and the Hitchin system for such a case has recently been studied for example in reference [60]. It is illuminating to consider the degrees of freedom visible only on the $\mathrm{SU}(2)$ component of the discriminant locus in the case that there are no $(\mathbf{6}, \mathbf{2})$ anti-symmetric fields. In this case $r=0$ and we have $n$ bifundamentals and $2 n+16$ fundamentals of SU(2). There are $4 n+22$ total moduli of the system, plus $3 n+18$ additional $\mathrm{SU}(2)$ singlets not visible from the $\mathrm{SU}(2)$ component of the discriminant (i.e. matter in the $(\mathbf{4}, \mathbf{1})$ and $(\mathbf{6}, \mathbf{1})$ representations). The previously mentioned example of a local -2 curve with $\mathrm{SU}(2)$ gauge theory corresponds to the special case where we take $n=-2$ and $r=0$. Note that in this case, we cannot retain the SU(4) enhancement locus. Indeed, otherwise some of the entries in table 1 would have negative multiplicity.

Let us now turn to the Hitchin system interpretation of this model. Assuming we have tuned the moduli of the Weierstrass model to have an $\mathrm{SU}(4) \times \mathrm{SU}(2)$ gauge theory, we see that we actually have two Hitchin systems which are coupled via the source terms provided by the localized matter. Additionally, we see that generically, different sorts of bifundamental representations will be present. Now, from the perspective of the parent gauge theory described in section 4, we also observe that poles in the Higgs field require background values for a single hypermultiplet. In other words, even if we try to tune the moduli so that different matter fields localize at the same point of a curve, there is no pairing available between different matter field representations. So in this sense, these tunings of different representations cannot change the pole order, but only the location of non-zero entries for each generalized residue.

Assuming that all such localized matter are kept at distinct points, we see that from the perspective of the $\mathrm{SU}(2)$ factor, we can engineer only simple poles. Even so, it should still be noted that in this case it is nonetheless possible to achieve a generic T-brane configuration for the $\mathrm{SU}(2)$ factor. Conversely, we also see that for the SU(4) factor, the presence of different representations, such as the $\mathbf{6}$ and $\mathbf{4}$, and the respective multiplicities allows us to fill out T-branes with simple poles.

Suppose next that we start to bring the various matter fields to the same location in the geometry. In this context it is possible to begin to engineer general wild T-brane solutions, embedded in $\mathrm{SU}(2) \times \mathrm{SU}(4)$. Using the techniques of section 2.1 to embed $\mathrm{SU}(2)$ Hitchin systems into large gauge groups, we see that depending on the choice of structure group of 
the Higgs bundle, a variety of breaking patterns are possible, including unbroken $6 \mathrm{D}$ symmetry $\mathrm{SU}(2) \times \mathrm{SU}(2), \mathrm{SU}(4)$, and $\mathrm{SU}(2)$ (as well as the maximally broken/unbroken cases).

We will consider several choices of Hitchin system which break some of the gauge symmetry of the $\mathrm{SU}(4) \times \mathrm{SU}(2)$ system. For ease of exposition, we shall first consider embedding a Higgs bundle into the SU(2) factor. Given a choice of $n$ and $r$, we would like to know, for example, the maximal pole order available in a wild SU(2) Hitchin system engineered in such a global model. If we choose to also break the SU(4) gauge symmetry (i.e. break the full gauge group), we can now expect that doublets of the SU(2) Higgs bundle which descend from different sources can all combine to produce a higher order pole. The total number of such doublets is:

$$
F_{\text {doub }}=3 r+(2 n+r+16)+4 n-4 r=6 n+16 .
$$

(note that as expected by anomaly cancellation, this is the same number of doublets arising in the $6 \mathrm{D}$ theory from a single, generic $\mathrm{SU}(2)$ symmetry on $\mathbb{F}_{n}[42]$ ). From this, we conclude that the highest order pole which can be achieved in such models is obtained by a maximal breaking pattern of the $\mathrm{SU}(4)$ factor, and with $\mathrm{SU}(2)$ Higgs bundle pole order:

$$
k_{\max }=6 n+16 .
$$

As a check, we will arrive at (5.10) in appendix $\mathrm{C}$ by geometric methods. For example, when $n=-2$, we can achieve a single pole of order 4 . We study the moduli space of wild Hitchin systems for this case in much greater detail in appendix A. At the absolute extreme where $n=12$, we can achieve a pole of order 88 .

Now, in contrast to the case above, let us consider embedding a Higgs bundle with structure group $\mathrm{SU}(2) \subset \mathrm{SU}(4)$ only. This choice leaves unbroken $\mathrm{SU}(2) \times \mathrm{SU}(2)$ in the $6 \mathrm{D}$ theory and gives rise to an anomaly consistent spectrum of $n(\mathbf{2}, \mathbf{2})$ bifundamentals and $4 n+16[(\mathbf{1}, \mathbf{2})+(\mathbf{2}, \mathbf{1})]$ representations. Here then the relevant counting of doublets "visible" to the SU(2) Higgs bundle is obtained by the branching rules of SU(4) $\rightarrow \mathrm{SU}(2)$ and the counting the original number of $\mathbf{4}$ and $\mathbf{6}$ multiplets in the CY geometry, leading to

$$
F_{\text {doub }}^{\prime}=r+(n-r+2)+(2 n+2 r+16)+2(n-r)=5 n+18 .
$$

In this case the maximal pole order is clearly different than the case above. For example, if only the location of the $(\mathbf{6}, \mathbf{2})$ and $(\mathbf{4}, \mathbf{2})$ multiplets are forced to overlap, then the maximal pole order in the $\mathrm{SU}(2) \subset \mathrm{SU}(4)$ Higgs bundle is determined by $2 n-r=2 k_{\max }$. In the case that $r=0$ we have the much more restrictive bound of $k_{\max }=n$. We provide these examples to illustrate that the for the same $C Y$ geometry there can be multiple choices of T-brane solutions, with different wild pole structure, leading to entirely different $6 \mathrm{D}$ effective theories.

In appendix $\mathrm{C}$, we will study bifundamentals associated with $\mathrm{SU}(M)-\mathrm{SU}(N)$ collisions from a geometric perspective. While we do not explicitly study collisions with additional matter as in the situation of table 1, more general situations can be understood in principle by combining the analyses of bifundamentals and fundamentals considered separately in appendix C. 


\section{$5.2 \quad$ Tangent bundle to $K 3$ revisited}

Most of the examples encountered so far in this paper have used an explicit weakly coupled hypermultiplet. Recent work on 6D superconformal field theories has also shown that the correspondence between matter and complex structure deformations also extends to the case of conformal matter as in references [35-38]. Here we study this phenomenon in one particularly tractable case: small instantons of heterotic theory which have been dissolved back into finite size instantons. We focus on the case of deformations to an unbroken $E_{7}$ gauge group, namely, the breaking pattern involves an $\mathrm{SU}(2)$ gauge theory. Our plan in this subsection will be to study this case in greater detail, following the analysis presented in reference [4] (see also [42, 61]).

In the spectral cover construction, or equivalently in the F-theory dual realization, we have a Hitchin system on a $\mathbb{P}^{1}$ with $12+n$ marked points, the number of instantons in the heterotic dual description. In the theory with a smoothing deformation, we have an $\mathfrak{e}_{7}$ singularity which degenerates to an $\mathfrak{e}_{8}$ singularity at marked points. The minimal Weierstrass model is:

$$
y^{2}=x^{3}+\varepsilon f_{8+n}(u) w^{3} x+g_{12+n}(u) w^{5},
$$

that is, we have $8+n$ half hypermultiplets in the $\mathbf{5 6}$ of $E_{7}$. The zeroes of $g_{12+n}(u)$ indicates the locations of the small instantons. Dissolving these small instantons into flux amounts to activating a source for the Higgs field, triggering a breaking pattern down to $E_{7}$. The parameter $\varepsilon$ indicates that we can also take a limit where we proceed back to a singular T-brane configuration.

We review and extend the description from [4] of this breaking pattern in terms of Hitchin systems. Let $D \subset \mathbb{P}^{1}$ be the divisor consisting of the $12+n$ zeros of $g_{12+n}$. There is essentially no change in our conclusions if some of the zeros of $g_{12+n}$ have multiplicity greater than one.

The breaking pattern from $E_{8}$ to $E_{7}$ is governed by an $\mathrm{SU}(2)$ Hitchin system. So, we consider meromorphic SU(2) Higgs bundles

$$
\Phi: E \rightarrow E \otimes K_{\mathbb{P}^{1}}(D)
$$

with simple poles on $D$. Here, if $p$ occurs in $D$ with multiplicity $m$, by a "simple pole on $D$ " we mean that the poles of $\Phi$ at $p$ can have order $m$. Our conclusion is that $\operatorname{Tr}\left(\Phi^{2}\right)$ also has simple poles on $D$, just as in the situation where the zeros of $g_{12+n}$ are isolated.

Next, consider the parameter space

$$
B=H^{0}\left(\mathbb{P}^{1}, K_{\mathbb{P}^{1}}^{2}(D)\right)
$$

Note that $\operatorname{dim} B=n+9$ when $m=n+12$. For each $b \in B$, we construct a spectral cover $C_{b}$ of $\mathbb{P}^{1}$ inside the total space of the line bundle $K_{\mathbb{P}^{1}}(D)$. Letting $y$ be a point of this total space, then the spectral cover $\pi: C_{b} \rightarrow \mathbb{P}^{1}$ is given by the equation

$$
y^{2}=g_{n+12} b .
$$

Note that $g_{n+12} b \in H^{0}\left(\mathbb{P}^{1}, K_{\mathbb{P}^{1}}^{2}(2 D)\right)$, so (5.15) makes sense. 
If we have a line bundle $L$ on $C_{b}$ (or torsion-free sheaf more generally), we recover a meromorphic Higgs bundle by putting $E=\pi_{*} L$. The embedding of $C_{b}$ in the total space of $K_{\mathbb{P}^{1}}(D)$ gives rise to $\Phi: E \rightarrow E \otimes K_{\mathbb{P}^{1}}(D)$ in the same manner as the case of a Higgs field without singularities.

Now $C_{b}$ is singular over the points $p \in \mathbb{P}^{1}$ at which $g_{n+12}$ vanishes to higher order. The important point for us is that these singularities do not affect the moduli count. This is because the tangent space to the moduli space $H^{1}\left(C_{b}, \mathcal{O}_{C_{b}}^{*}\right)$ of line bundles on $C_{b}$ is $H^{1}\left(C_{b}, \mathcal{O}_{C_{b}}\right)$. Since $C_{b}$ is connected, the dimension of this vector space is just the arithmetic genus of $C_{b}$, which is a deformation invariant so can be computed by Riemann-Hurwitz by assuming that the zeros of $g_{n+12}$ are distinct. The result is that the genus, hence the dimension, is $n+9$.

There are $n+12$ additional pairs of moduli. The moduli of $B$ can be enlarged by letting the points of $D$ vary, i.e. the base space can be enlarged to a fibration over $S y m^{n+12} \mathbb{P}^{1}$ with typical fiber $B$. The other half of the pair of $n+12$ moduli appear in what are identified with RR moduli in the IIA description, arising from the limiting mixed Hodge structure.

\section{Conclusions}

A central pillar of F-theory is the close correspondence between the geometry of elliptically fibered Calabi-Yau manifolds, and the resulting moduli space of vacua for the low energy effective field theory. Vacua with T-branes generalize this correspondence since they involve non-abelian intersections of 7-branes which are not visible in complex geometry. In this paper we have shown that the open string patch of this moduli space is governed by a Hitchin system coupled to defects. Non-zero background values for these defects provide a systematic way to build up T-branes localized at points as well as non-reduced schemes. This gives a systematic method for engineering wild Higgs bundles in F-theory. CalabiYau geometry also unifies seemingly different wild Hitchin systems: changes in the order of poles or in the nature of residues are captured by corresponding variations in the gauge singlet complex structure moduli of an F-theory compactification. Constraints from 6D effective field theory translate to geometric conditions on the order of poles, and the rank of generalized residues which can actually be realized by an F-theory model. In the remainder of this section we discuss some avenues for future investigation.

An implicit feature of the results in this paper is the way in which limiting mixed Hodge structure is completed in singular limits (as in (1.1)) by an emergent Hitchin system [4]. It would be instructive to develop this explicit correspondence in more detail, both as a general "proof of principle" as well as a means to further unify a priori distinct wild Hitchin systems.

Along these lines, wild Hitchin systems in F-theory must incorporate a sufficient number of charged matter fields to realize certain types of higher order poles. A natural question is whether there is a universal maximum upper bound possible in global models. Using the results of this paper, we see that this question translates into determining a sharp upper bound on the total amount of matter which can be charged under any particular gauge group in an F-theory model. 
Another feature of wild Higgs bundles is the necessity of introducing Stokes chambers in order to properly account for the generalized monodromy experienced by holomorphic sections of such singular bundles. Returning to the geometry of an F-theory compactification, we expect that upon further compactification on a $T^{2}$ that such holomorphic sections will correspond to matter fields localized at a point of the $T^{2}$ factor and spread over the Hitchin system curve in question. Since such matter fields also correspond to localized deformations of the complex structure moduli of the Calabi-Yau, this also suggests that the notion of Stokes chambers and generalized monodromy matrices should also lift to the complex structure moduli and intermediate Jacobian of the Calabi-Yau threefold. Developing the resulting effect on the periods of holomorphic three-forms with higher order singularities - namely not just simple poles along a divisor — would be most instructive.

Now that we have a clear interpretation of most Hitchin system phenomena in terms of their corresponding F-theory avatars, it is natural to consider next the resulting 4D vacua obtained from compactification on a Calabi-Yau fourfold. The reduced supersymmetry means that we should not expect the moduli space of vacua to define a hyperkahler manifold. Nevertheless, we expect the methods developed here and in [4] to persist in this more general setting.

Finally, one of the important phenomenological applications of T-branes is in the construction of realistic Yukawa couplings. To extract these couplings, it is necessary to know the localized profile of matter field wave functions. We have also seen that the presence of higher order singularity types has an impact on the local presentation of matter fields, since the actual normalizable wave functions depend on Stokes data. It would be interesting to study the possible impact on wave function profiles, as well as the overlap of multiple wave functions.

\section{Acknowledgments}

We thank P. Boalch, S. Gukov, R. Donagi, J. Hurtubise, D.R. Morrison, S. Rayan, and W. Taylor for helpful discussions. JJH thanks the 2015 Simons Center Summer Workshop on Geometry and Physics for kind hospitality during part of this work. SK thanks UNC Chapel Hill and the organizers of the Spring 2015 southeast conference on string theory for kind hospitality during the completion of part of this work. LBA, JJH, SK and LPS thank the organizers of the 2015 FRG workshop at Harvard for providing a stimulating atmosphere for collaboration. The work of LBA is supported by NSF grant PHY-1417337 and is a part of the working group activities of the 4-VA initiative "A Synthesis of Two Approaches to String Phenomenology." The work of JJH is supported by NSF CAREER grant PHY-1452037. JJH also acknowledges support from the Bahnson Fund at UNC Chapel Hill. The work of SK was supported by NSF grants DMS-1201089 and DMS1502170. The work of LPS is supported by NSF grant DMS 1509693, and she would like to thank the hospitality of IMPA, Brazil, and the support of the GEAR Network through NSF grants DMS 1107452, 1107263, 1107367. 


\section{A Introduction to wild Hitchin systems}

In this appendix we present some of the salient features of wild Higgs bundles. Our aim here will be to give a self-contained introduction to those aspects relevant for our discussion of wild T-branes in F-theory. We shall be interested in the Hitchin system on a genus $g$ curve $C$, for some gauge group $G$ (see [62-64] and references therein for relevant background material). In the physics literature, it is common to reference a gauge group $G$, an adjoint valued $(1,0)$ form $\Phi$, and a gauge connection $A$. It is convenient to instead work in terms of the pair $(E, \Phi)$, where $E$ is a stable holomorphic vector bundle with structure group $G$.

In order to describe the basic geometric features in the study of wild Higgs bundles, it is enough to limit ourselves to Higgs bundles $(E, \Phi)$ over the projective line $\mathbb{P}^{1}$. This is an effective divisor $D$, a vector bundle $\mathcal{E}$ of rank $n$; and an $K_{\mathbb{P}^{1}}(D)$ valued endomorphism $\Theta: \mathcal{E} \rightarrow \mathcal{E} \otimes K_{\mathbb{P}^{1}}(D)$ called the Higgs field. The divisor $D$ controls where $\Phi$ is allowed to have poles, so it is sometimes referred to as the polar divisor.

It is useful to suppose that there is a compatible parabolic structure on $p_{j}$, in the sense of Mehta and Seshadri. This means, a set of parabolic weights which are real numbers

$$
0 \leq \alpha_{0}^{j} \leq \ldots \leq \alpha_{r-1}^{j}<1,
$$

and a finite decreasing filtration $\{0\}=\left(\mathcal{E}_{p_{j}}\right)_{1} \subseteq\left(\mathcal{E}_{p_{j}}\right)_{\alpha_{r-1}^{j}} \subseteq \ldots \subseteq\left(\mathcal{E}_{p_{j}}\right)_{0}=\mathcal{E}_{p_{j}}$ of the fibre of $\mathcal{E}$ at $p_{j}$ preserved by the residue $\operatorname{res}\left(\Phi, p_{j}\right)$, where $r_{j}$ is the smallest index such that $\alpha_{r_{j}}^{j}>0$ (when such an index exists; $r_{j}=r$ otherwise). In what follows, we shall consider particular examples to illustrate the theory that has been developed in the literature to study Higgs bundles with poles.

We adopt the notation of reference [65] where a wild Higgs bundle of type $\left(m, r_{1}, r_{2}, \ldots\right)$ is a Higgs bundle with $m$ poles of orders $k_{i}:=r_{i}+1$. Then, as explained in [65, Remark 9.12] for rank two Higgs bundles, one obtains moduli spaces of complex dimension two when

$$
\left(m, r_{1}, r_{2}, \ldots\right)=(4,0,0,0,0),(3,1,0,0),(2,1,1),(2,2,0),(1,3),
$$

denoting four poles of order 1 ; two of order 2 and two poles of order 1 ; or two poles of order 2; or two poles of order 3 and a simple pole; or finally one pole of order 4 .

A comment on notation. In the paper, and in particular in section 4, when considering Higgs bundles with a single pole of order $k$ at zero, it is convenient to adopt similar notation to that used in [44, eq. (1.1)] where the corresponding complexified connection is written as

$$
\mathcal{A}=d u\left(\frac{T_{k}}{u^{k}}+\ldots+\frac{T_{2}}{u^{2}}+\frac{T_{1}}{u}\right)
$$

where $T_{1}, T_{2}, \ldots, T_{k}$ are elements of the Lie algebra of group. On the other hand, in this appendix we would like to review some known results for low rank Higgs bundles (see e.g. [56]). To this end, we now write a complexified connection as:

$$
\mathcal{A}=d Q+T_{1} \frac{d u}{u},
$$

for $Q$ a square matrix of meromorphic connections. From the above papers, one has the following definition. 
Definition A.1 The formal monodromy of $\mathcal{A}$ is $M_{0}:=e^{2 \pi i T_{1}}$. Moreover, we say that $T_{1}$ is the exponent of the formal monodromy.

One should keep in mind that varying $Q$ from the perspective of reference [56], will be considered equivalent to varying $\left(T_{k}, T_{k-1}, \ldots, T_{2}\right)$ from the perspective [44].

In what follows, we shall give a detailed description of data associated to Wild Higgs bundles of type $(1,1)$, and build up to two of the cases where the moduli space has dimension 2 : the ones of $(1,3)$ and $(2,1,1)$ following closely [66] and references therein.

When calculating moduli dimensions, as done as in [65, Remark 9.12] and references therein, one needs to consider isomonodromic deformation [45], given by meromorphic differential equations by which one can vary the parameters contained in $\left(T_{k}, T_{k-1}, \ldots, T_{2}\right)$ without changing the generalized monodromy $\widehat{M}$ obtained as the product of the formal monodromy and the Stokes matrices (e.g. see [44, eq. (2.29)]).

Rank 2 Higgs bundles with 1 pole of order 2. We will follow [56] to study Higgs bundles $(E, \Phi)$ on $\mathbb{P}^{1}$ of rank $r=2$ and with only one pole of order 2 in one marked point $p_{1}=0$. This is equivalent to considering a diagonal generic meromorphic connection $d-\mathcal{A}$ on the trivial rank 2 vector bundle over the unit disc $\mathbb{D} \subset \mathbb{C}$ with only a pole of order 2 at 0 . For $u$ a coordinate vanishing at 0 , we write

$$
\mathcal{A}=d Q+T_{1} \frac{d u}{u}
$$

where $T_{1}$ is a constant diagonal matrix, and $Q$ is a diagonal matrix of meromorphic functions $[56, \mathrm{p} .154] .{ }^{10}$ We write the complexified connection as:

$$
\mathcal{A}=d u\left(\frac{T_{2}}{u^{2}}+\frac{T_{1}}{u}\right)
$$

Hence, in terms of [44], one is taking $T_{2}=\frac{d Q}{d u} u^{2}$. Considering $\widetilde{q}_{i j}$ as in [44, eq. (2.11)] without the tilde, this matrix is

$$
T_{2}=\left(\begin{array}{cc}
\widetilde{q}_{12} & 0 \\
0 & \widetilde{q}_{22}
\end{array}\right) .
$$

One should note that along [44], when the order $k$ of the single pole is fixed, the notation is simplified and the diagonal entries of $T_{k}$ are denoted by $\widetilde{q}_{j}$ with no tilde. For convenience we shall follow the notation in [56] appearing in (A.4). In particular, we have

$$
Q=\left(\begin{array}{cc}
q_{1}(u) & 0 \\
0 & q_{2}(u)
\end{array}\right)
$$

and define $q_{i j}(u)$ as the leading term of $q_{i}(u)-q_{j}(u)$. Letting $q_{1}(u)-q_{2}(u)=a / u+b$, then

$$
q_{12}(u)=a / u, \text { and } q_{21}(u)=-a / u .
$$

\footnotetext{
${ }^{10}$ Note that $Q$ is determined by $\mathcal{A}$ and $u$ by requiring that it has constant term zero in its Laurent expansion with respect to $u$.
} 
Note that from (A.5)-(A.6) one has that $\widetilde{q}_{i}=q_{i}^{\prime}(u) u^{2}$, and hence $\widetilde{q}_{i}-\widetilde{q}_{j}=-a$. In what follows we shall use polar coordinates and write $a=\widetilde{r} e^{i \widetilde{\theta}}$.

Associated to the above connections are anti-Stokes directions, which can be obtained as follows. Consider directed lines in $\mathbb{C}$ through the origin parameterized by $S^{1}$. For $\mathrm{d}_{1}, \mathrm{~d}_{2} \in S^{1}$ we let $\operatorname{Sect}\left(\mathrm{d}_{1}, \mathrm{~d}_{2}\right)$ be the (open) sector swept out by rays rotating from the point $\mathrm{d}_{1}$ to the point $\mathrm{d}_{2}$.

Definition A.2 The anti-Stokes directions $\mathbb{A} \subset S^{1}$ obtained through the matrix $Q$ are the directions $d \in S^{1}$ for which either

$$
q_{12}(u) \in \mathbb{R}_{<0}, \quad \text { or } \quad q_{21}(u) \in \mathbb{R}_{<0}
$$

for $u$ on the ray specified by $d$.

These are the directions along which $e^{(a / u+b)}$ (respectively $e^{-(a / u+b)}$ ) decays most rapidly as $u$ approaches 0 (note that $\mathbb{A}$ is independent of the coordinate choice $u$ ). Moreover, these directions have $\pi$ rotational symmetry: if $\pm \frac{a}{u} \in \mathbb{R}_{<0}$ then $\mp \frac{a}{u} e^{-i \pi} \in \mathbb{R}_{<0}$, and thus $\# \mathbb{A}:=2$. Note that if $q_{12}(u) \in \mathbb{R}_{<0}$ for a direction $d$, then $q_{21}(u) \notin \mathbb{R}_{<0}$ for that direction (and vice versa), and thus in this set up, a direction is referred to as a half-period.

Definition A.3 For $d \in \mathbb{A}$ an anti-Stokes direction, the roots of $d$ are

$$
\operatorname{Roots}(d):=\left\{(i j) \mid q_{i j}(u) \in \mathbb{R}_{<0} \text { along } d\right\} .
$$

Remark A.4 In order to find the directions, for one single pole of order $k$ we must study angles for which $a / u^{k-1}$ is negative. For $a=\widetilde{r} e^{i \widetilde{\theta}}$ as before, we need $u=r e^{i \theta}$ for which $\widetilde{\theta}-(k-1) \theta=(2 m+1) \pi$ for $m \in \mathbb{N}$. In such a case, one has that $a / u^{k-1}=-\widetilde{r} / r^{k-1}$ which is negative. Moreover, in general we shall call a half-period an l-tuple $\mathrm{d}=\left(\mathrm{d}_{1}, \ldots, \mathrm{d}_{l}\right) \subset \mathbb{A}$ of consecutive anti-Stokes directions, where $2 l=\# \mathbb{A}$. When weighted by their multiplicities, the number of anti-Stokes directions in any half-period is $1=\operatorname{Mult}\left(\mathrm{d}_{1}\right)+\ldots+\operatorname{Mult}\left(\mathrm{d}_{l}\right)$.

In our case there are only two half periods (see figure 4 ):

- One supported by $q_{12}(u)$ in a direction $\mathrm{d}_{1}:=e^{i(\widetilde{\theta}-\pi)}$, for which taking $u=r e^{i(\widetilde{\theta}-\pi)}$ one has $q_{12}(u)=-\frac{\widetilde{r}}{r} \in \mathbb{R}_{<0}$;

- One supported by $q_{21}(u)$ in a direction $\mathrm{d}_{2}:=e^{-i \pi} \mathrm{d}_{1}=e^{i \theta}$, for which taking $z=r e^{i \theta}$ one has $q_{21}(u)=-\frac{\widetilde{r}}{r} \in \mathbb{R}_{<0}$.

Moreover, from Definition A.3, in the current setting one has

$$
\operatorname{Roots}\left(\mathrm{d}_{1}\right)=\{(12)\}, \text { and } \operatorname{Roots}\left(\mathrm{d}_{2}\right)=\{(21)\} \text {. }
$$

Definition A.5 The multiplicity Mult $(d)$ of $d$ is the number of roots supporting $d$, which from Definition A.3 is 1. Finally, the group of Stokes factors ${ }^{11}$ associated to $d$ is the group

$$
\mathbb{S t o}_{d}(\mathcal{A}):=\left\{K \in G \mid(K)_{i j}=\delta_{i j} \text { unless }(i j) \text { is a root of } d\right\},
$$

which is a unipotent subgroup of $G=\mathrm{GL}(2, \mathbb{C})$ of dimension equal to $\operatorname{Mult}(d)=1$.

\footnotetext{
${ }^{11}$ Note that these are called Stokes matrices in [67] and referred to as $S t_{d}$.
} 


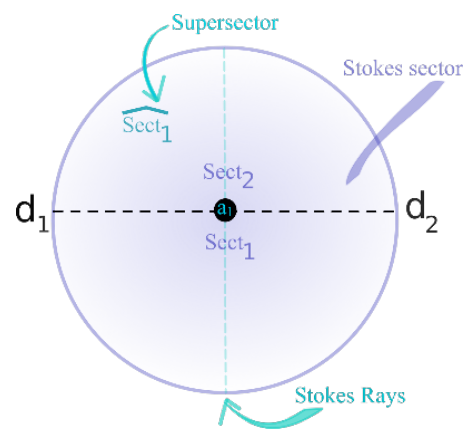

Figure 3. Stokes rays, and Stokes Sectors and Super Sectors.

For both directions $\mathrm{d}_{1}$ and $\mathrm{d}_{2}$ that one has, the group of Stokes factors is a 1dimensional subgroup given by

$$
\mathbb{S t o}_{\mathrm{d}_{1}}(\mathcal{A})=\left\{\left(\begin{array}{ll}
1 & \widetilde{u} \\
0 & 1
\end{array}\right) \text { for } \widetilde{u} \in \mathbb{C}\right\} \text { and } \mathbb{S t o}_{\mathrm{d}_{2}}(\mathcal{A})=\left\{\left(\begin{array}{cc}
1 & 0 \\
\widetilde{u} & 1
\end{array}\right) \text { for } \widetilde{u} \in \mathbb{C}\right\}
$$

In particular, note that this agrees with Lemma 3.2 of [56] since, as a variety,

$$
\begin{aligned}
\operatorname{Sto}_{\mathrm{d}_{1}}(\mathcal{A}) \cdot \mathbb{S t o}_{\mathrm{d}_{2}}(\mathcal{A}) & \cong U_{+} \times U_{-} \\
\left(K_{1}, K_{2}\right) & \mapsto\left(S_{1}, S_{2}\right)
\end{aligned}
$$

for $U_{ \pm}$the lower and upper triangular unipotent subgroups of $\mathrm{GL}(2, \mathbb{C})$, and

$$
S_{1}:=K_{1} K_{2} \text {, and } S_{2}:=K_{2} K_{1} \text {. }
$$

We will see later that these are the Stokes matrices, and are each in $U_{ \pm}$respectively (but one needs to be careful with the above double lower index). Each of the two Stokes directions defines an ordering: $q_{1}<_{\mathrm{d}_{1}} q_{2}$, and $q_{2}<_{\mathrm{d}_{2}} q_{1}$. In particular one has that

$$
\begin{array}{ll}
e^{q_{1}} / e^{q_{2}} \rightarrow 0 \text { along the ray } \theta_{1}:=e^{i \frac{3 \pi}{2}} \in S^{1} \quad \text { bisecting Sect } 1:=\operatorname{Sect}\left(\mathrm{d}_{1}, \mathrm{~d}_{2}\right), \\
e^{q_{2}} / e^{q_{1}} \rightarrow 0 \text { along the ray } \theta_{2}:=e^{i \frac{\pi}{2}} \in S^{1} \quad \text { bisecting Sect } 2:=\operatorname{Sect}\left(\mathrm{d}_{2}, \mathrm{~d}_{1}\right)
\end{array}
$$

and these rays are called Stokes Rays as seen in figure 3, and the sectors bounded by the Stokes rays are referred to as super sectors $\widehat{\text { Sect. }}$

Remark A.6 (Parameter space) One should note that from (A.4) the only parameters in all the above considerations are the constant $2 \times 2$ diagonal matrix $T_{1}$, and the diagonal matrix $Q$. The latter, from (A.7), is equivalent to the parameters a and $b$ for which $q_{1}(u)-$ $q_{2}(z)=a u+b$.

We shall now consider the local moduli of meromorphic connections. For this, let $\operatorname{Sys}(\mathcal{A})$ be the set of germs at $0 \in \mathbb{C}$ of meromorphic connections on the rank 2 bundle that are formally equivalent to $d-\mathcal{A}$ (as in (A.4)). Formally,

$$
\operatorname{Syst}(\mathcal{A})=\{d-A \mid A=\widehat{F}[\mathcal{A}] \text { for some } \widehat{F} \in G[[z]]\},
$$


where $A$ is a matrix of germs of meromorphic one-forms, $G[[u]]=\mathrm{GL}(2, \mathbb{C}[[u]])$ and

$$
\widehat{F}[\mathcal{A}]=(d \widehat{F}) \widehat{F}^{-1}+\widehat{F} \mathcal{A} \widehat{F}^{-1}
$$

For $G\{u\}:=\operatorname{GL}(2, \mathbb{C}\{u\})$ the subgroup of germs of holomorphic bundle automorphisms, we are interested in $\operatorname{Sys}(\mathcal{A}) / G\{u\}$. For Higgs bundles of rank 1 or with just one pole, this is just a point. Since we are considering here rank 2 Higgs bundles with one single double pole, in this case the group is non-trivial and it can be described in terms of Stokes matrices. We shall denote by $\widehat{\operatorname{Sys}}_{c f}(\mathcal{A})$ the set of compatibly framed connection germs with both irregular and formal type $\mathcal{A}$.

Definition A.7 The set $\widehat{\operatorname{Sys}}(\mathcal{A})_{\text {cf }}$ is isomorphic to the set of marked pairs, defined as

$$
\widehat{\operatorname{Sys}}(\mathcal{A})_{m p}:=\{(A, \widehat{F}) \mid A \in \operatorname{Sys}(\mathcal{A}), \widehat{F} \in G[[u]], A=\widehat{F}[\mathcal{A}]\} .
$$

Remark A.8 We can think of $\widehat{\operatorname{Sys}}(\mathcal{A})_{c f}$ as the set of pairs $\left(A, g_{0}\right)$ with $A \in \operatorname{Sys}(\mathcal{A})$ and $g_{0} \in G$ such that $g_{0}[A]$ and $\mathcal{A}$ have the same leading term.

Note that the above correspondence ${ }^{12}$ can be seen by taking $g_{0}:=\widehat{F}(0)^{-1}$. Then, one has the quotient $\mathcal{H}(\mathcal{A})=\widehat{\operatorname{Sys}}(\mathcal{A}) / G\{u\}$, and for $T \cong\left(\mathbb{C}^{*}\right)^{2}$ it follows that $\operatorname{Sys}(\mathcal{A}) / G\{u\} \cong$ $\mathcal{H}(\mathcal{A}) / T$. We shall consider the following sectors Sect and super sectors $\widehat{\text { Sect: }}$

$$
\begin{aligned}
& \operatorname{Sect}_{1}:=\operatorname{Sect}\left(e^{i(\widetilde{\theta}-\pi)}, e^{i \theta}\right) \\
& \operatorname{Sect}_{2}:=\operatorname{Sect}\left(e^{i \theta}, e^{i(\widetilde{\theta}-\pi)}\right)\left(:=\operatorname{Sect}_{0}\right) \\
& \widehat{\operatorname{Sect}}_{1}:=\operatorname{Sect}\left(e^{i\left(\widetilde{\theta}-\frac{3}{2} \pi\right)}, e^{i\left(\widetilde{\theta}+\frac{1}{2} \pi\right)}\right) \ni \mathrm{d}_{1} \\
& \widehat{\operatorname{Sect}}_{2}:=\operatorname{Sect}\left(e^{i\left(\widetilde{\theta}-\frac{1}{2} \pi\right)}, e^{i\left(\widetilde{\theta}-\frac{1}{2} \pi\right)}\right) \ni \mathrm{d}_{2}
\end{aligned}
$$

Note that given $\widehat{F}$ a formal transformation such that $A:=\widehat{F}[\mathcal{A}]$, there are canonical $2 \times 2$ matrices of holomorphic functions

$$
\begin{array}{lll}
\Sigma_{1}(\widehat{F}) \text { on Sect } 1 \text { such that } & \Sigma_{1}(\widehat{F})[\mathcal{A}]=A, \\
\Sigma_{2}(\widehat{F}) \text { on } \text { Sect }_{2} \text { such that } & \Sigma_{2}(\widehat{F})[\mathcal{A}]=A,
\end{array}
$$

defined uniquely such that $\Sigma_{j}(\widehat{F})$ can be analytically extended to $\widehat{\operatorname{Sect}}_{j}$, and such that it is asymptotic to $\widehat{F}$ on 0 within $\widehat{\operatorname{Sect}}_{j}$. For a fixed $p \in \operatorname{Sect}_{2}$, choose a branch of $\log (u)$ giving a lift $\widetilde{p}$ of $p$ to the universal cover of the punctured disc $\mathbb{D}_{0}$. Then, the canonical fundamental solution of $A$ on the sectors Sect $_{1}$ and Sect $_{2}$ are

$$
\begin{aligned}
\Phi_{1} & =\Sigma_{1}(\widehat{F}) u^{T_{1}} e^{Q}, \\
\Phi_{2} & =\Sigma_{2}(\widehat{F}) u^{T_{1}} e^{Q},
\end{aligned}
$$

\footnotetext{
${ }^{12}$ The matrix entries $\widehat{F}_{i j}$ are 0 if $i \neq j$, and constant if $i=j$.
} 
respectively, for a $\operatorname{fixed}^{13}(\mathcal{A}, z, \widetilde{p})$ and $\left(A, g_{0}\right) \in \widehat{\operatorname{Syst}}(\mathcal{A})$ (as in Remark A.8). For this fixed data, the Stokes factors are

$$
\begin{aligned}
& K_{1}=e^{-Q} u^{-T_{1}} \kappa_{1} u^{T_{1}} e^{Q}, \\
& K_{2}=e^{-Q} u^{-T_{1}} \kappa_{2} u^{T_{1}} e^{Q},
\end{aligned}
$$

where the $\kappa_{1}, \kappa_{2}$ are the matrix of holomorphic functions defined by

$$
\kappa_{1}=\Sigma_{1}(\widehat{F})^{-1} \circ \Sigma_{2}(\widehat{F}), \text { and } \kappa_{2}=\Sigma_{2}(\widehat{F})^{-1} \circ \Sigma_{1}(\widehat{F}) .
$$

The Stokes matrices are essentially the transition matrices between the canonical fundamental solution $\Phi_{2}$ on Sect S $_{0}$ and $\Phi_{1}$ on the opposite sector, Sect ${ }_{1}$, when they are continued along the two possible paths in the punctured disk joining these sectors. Moreover,

$$
\Phi_{1}=\Phi_{2} \cdot P S_{-} P^{-1} \text { and } \Phi_{2}=\Phi_{1} \cdot P S_{+} P^{-1} M_{0}
$$

where $P_{a b}=\delta_{c(a) b}$ for $c$ the permutation of $\{1,2\}$ giving the ordering of the sector taken. (so it is the identity matrix if we consider the sector giving the ordering $q_{1}<q_{2}$ ).

Remark A.9 Whilst understanding tentacles will be most useful when many poles are considered at the same time, we should note that if one needed to define a tentacle involving poles of order two, the extra data needed would be two points $\mathrm{b}_{1}$ and $\mathrm{b}_{2}$ in red in figure 6 .

Rank 2 Higgs bundles with 1 pole of order 4. We shall focus here on Higgs bundles $(E, \Phi)$ on $\mathbb{P}^{1}$ with one pole of order 4 . Consider a diagonal generic meromorphic connection $d-\mathcal{A}$ on the trivial rank 2 vector bundle over the unit disc $\mathbb{D} \subset \mathbb{C}$ with a pole of order 4 at 0 and no others. For $u$ a coordinate vanishing at 0 , as in (A.4) we write

$$
\mathcal{A}=d Q+T_{1} \frac{d u}{u}
$$

where $T_{1}$ is a constant diagonal matrix, and $Q$ is a diagonal matrix of meromorphic functions. In terms of the notation of section 4 In particular, we have

$$
Q=\left(\begin{array}{cc}
q_{1}(u) & 0 \\
0 & q_{2}(u)
\end{array}\right)
$$

and define $q_{i j}(u)$ as the leading term of $q_{i}(u)-q_{j}(u)$. Letting

$$
q_{1}(u)-q_{2}(u)=\frac{a}{u^{3}}+\frac{b}{u^{2}}+\frac{c}{u}+d
$$

then $q_{12}(u)=\frac{a}{u^{3}}$ and $q_{21}(u)=-\frac{a}{u^{3}}$. We shall use polar coordinates and consider $a=\widetilde{r} e^{i \widetilde{\theta}}$. As before, the anti-Stokes directions $\mathbb{A} \subset S^{1}$ are the directions $d \in S^{1}$ for which either

$$
q_{12}(u) \in \mathbb{R}_{<0}, \quad \text { or } q_{21}(u) \in \mathbb{R}_{<0}
$$

\footnotetext{
${ }^{13}$ Recall that from (A.4) fixing $\mathcal{A}$ is equivalent to fix $Q$ and $T_{1}$. To this data that was already fixed, one is adding the data of Remark A.8 and a point $p$ with its lift $\widetilde{p}$, the latter being equivalent to the point $b_{1}$ in figure 5 .
} 
for $u$ on the ray specified by $d$. These have $\pi / 3$ rotational symmetry, since if $q_{i j}(u) \in \mathbb{R}_{<0}$ then $\left.q_{j i}\left(u e^{\frac{\pi i}{3}}\right)\right) \in \mathbb{R}_{<0}$. In particular, this says that the number $r$ of anti-Stokes directions is divisible by 6 , and so we define $l=r / 6$. An $l$-tuple $\mathbf{d}=\left(\mathrm{d}_{1}, \mathrm{~d}_{2}, \ldots, \mathrm{d}_{l}\right)$ of consecutive anti-Stokes directions is a half period, and since

$$
1=\operatorname{Mult}\left(\mathrm{d}_{1}\right)+\ldots+\operatorname{Mult}\left(\mathrm{d}_{l}\right)
$$

and multiplicities (number of roots supporting the direction $d$ ) are positive integers, one has that $l=1$ and hence there are 6 anti-Stokes directions. Each of these directions form what is called half-period. The 6 anti-Stokes directions can be described as follows:

- Three directions are supported by $q_{12}(u)=\widetilde{r} e^{i \widetilde{\theta}} / u^{3}$. For $j=1,2,3$, the directions are $\mathrm{d}_{12}^{j}:=e^{i\left(\frac{\tilde{\theta}}{3}-\frac{(2 j+1) \pi}{3}\right)}$, for which taking $u=r e^{i\left(\frac{\tilde{\theta}}{3}-(2 j+1) \frac{\pi}{3}\right)}$ one has

$$
q_{12}(u)=-\frac{\widetilde{r}}{r^{3}} \in \mathbb{R}_{<0}
$$

- Three directions are supported by $q_{21}(u)=-\widetilde{r} e^{i \widetilde{\theta}} / u^{3}$, defined as $\mathrm{d}_{21}^{j}:=e^{\frac{i \pi}{3}} \mathrm{~d}_{12}^{j}$ or equivalently $\mathrm{d}_{21}^{j}=e^{i\left(\frac{\tilde{\theta}}{3}-\frac{2 j \pi}{3}\right)}$, for which taking $u=e^{i\left(\frac{\tilde{\theta}}{3}-\frac{2 j \pi}{3}\right)}$ one has

$$
q_{21}(u)=-\frac{\widetilde{r}}{r^{3}} \in \mathbb{R}_{<0}
$$

and they appear in figure 4, agreeing with Definition A.2. Recall from Definition A.5 that the group of Stokes factors associated to a direction $d$ is the group

$$
\mathbb{S t o}_{d}(\mathcal{A}):=\left\{K \in G \mid(K)_{i j}=\delta_{i j} \text { unless }(i j) \text { is a root of } d\right\}
$$

which is a unipotent subgroup of $G=\mathrm{GL}(2, \mathbb{C})$ of dimension 1 . For all directions $\mathrm{d}_{12}^{j}$ and $\mathrm{d}_{21}^{j}$ that one has in this setting, for $j=1,2,3$, the group of Stokes factors is a 1-dimensional subgroup given by

$$
\begin{aligned}
& \mathbb{S t o}_{\mathrm{d}_{12}^{j}}(\mathcal{A})=\left\{\left(\begin{array}{ll}
1 & \widetilde{z} \\
0 & 1
\end{array}\right) \text { for } \widetilde{z} \in \mathbb{C}\right\} \\
& \mathbb{S t o}_{\mathrm{d}_{21}^{j}}(\mathcal{A})=\left\{\left(\begin{array}{ll}
1 & 0 \\
\widetilde{u} & 1
\end{array}\right) \text { for } \widetilde{u} \in \mathbb{C}\right\}
\end{aligned}
$$

In particular, this agrees with Lemma 3.2 (2) of [56] since, as a variety,

$$
\begin{aligned}
\Pi_{j=1}^{3}\left(\mathbb{S t o}_{\mathrm{d}_{12}^{j}}(\mathcal{A}) \cdot \mathbb{S t o}_{\mathrm{d}_{21}^{j}}(\mathcal{A})\right) & \cong\left(U_{+} \times U_{-}\right)^{3} \\
\left(K_{1}, \ldots, K_{6}\right) & \mapsto\left(S_{1}, \ldots, S_{6}\right)
\end{aligned}
$$

for $U_{ \pm}$the lower and upper triangular unipotent subgroups of $\mathrm{GL}(2, \mathbb{C})$, and

$$
S_{i}:=K_{i} K_{2(i-1)} \in U_{+/-} \text {for } i \text { odd/even }
$$




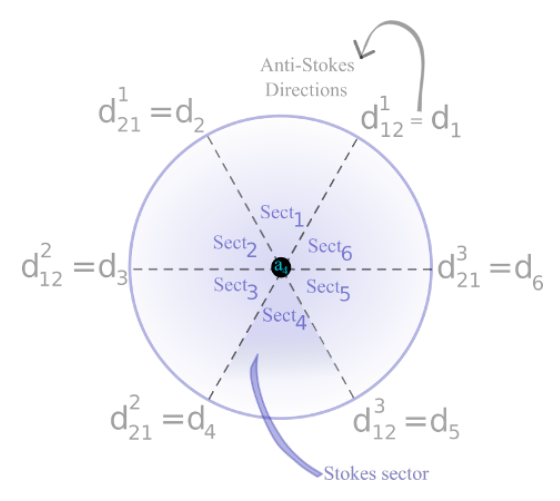

Figure 4. Anti-Stokes directions.

the Stokes matrices. Note that, independently of $j$, as in the previous case the Stokes directions $\mathrm{d}_{12}^{j}$ and $\mathrm{d}_{21}^{j}$ define the orderings $q_{1}<_{\mathrm{d}_{12}^{j}} q_{2}$, and $q_{2}<_{\mathrm{d}_{21}^{j}} q_{1}$. Recall that for a rank 2 Higgs bundle with one pole of order 2 one had two sectors defined as in (A.15)-(A.16). In this case, with only one pole of order 4 there are 6 sectors, $\operatorname{Sect}_{\mathrm{i}}:=\operatorname{Sect}\left(\mathrm{d}_{i}, \mathrm{~d}_{i+1}\right)$, with indices mod 6 as in figure 4 .

In order to consider the local moduli of meromorphic connections, let $\operatorname{Sys}(\mathcal{A})$ be the set of germs at $0 \in \mathbb{C}$ of meromorphic connections on the rank 2 bundle that are formally equivalent to $d-\mathcal{A}$. Formally,

$$
\operatorname{Syst}(\mathcal{A})=\{d-A \mid A=\widehat{F}[\mathcal{A}] \text { for some } \widehat{F} \in G[[u]]\},
$$

where $A$ is a matrix of germs of meromorphic one-forms, $G[[u]]=\mathrm{GL}(2, \mathbb{C}[[u]])$ and

$$
\widehat{F}[\mathcal{A}]=(d \widehat{F}) \widehat{F}^{-1}+\widehat{F} \mathcal{A} \widehat{F}^{-1} .
$$

As in the previous cases, one has $\widehat{\operatorname{Sys}}_{c f}(\mathcal{A})$ the set of compatibly framed connection germs with both irregular and formal type $\mathcal{A}$. The set $\widehat{\operatorname{Sys}}(\mathcal{A})_{c f}$ is isomorphic to the set of marked pairs be

$$
\widehat{\operatorname{Sys}}(\mathcal{A})_{m p}:=\{(A, \widehat{F}) \mid A \in \operatorname{Sys}(\mathcal{A}), \widehat{F} \in G[[u]], A=\widehat{F}[\mathcal{A}]\}
$$

Consider $\widehat{F}$ a formal transformation such that $A:=\widehat{F}[\mathcal{A}]$ has convergent series. From [56, Theorem 3.1] there are canonical $2 \times 2$ matrices of holomorphic functions on each Sect $_{j}$ given by $\Sigma_{j}(\widehat{F})$ on $\operatorname{Sect}_{i}$ such that $\Sigma_{j}(\widehat{F})[\mathcal{A}]=A$, defined uniquely such that $\Sigma_{j}(\widehat{F})$ can be analytically extended to $\widehat{\operatorname{Sect}}_{j}$, and such that it is asymptotic to $\widehat{F}$ on 0 within $\widehat{\operatorname{Sect}}_{j}$.

Remark A.10 As explained in [56], of all the holomorphic isomorphisms between $\mathcal{A}$ and $A$ which are asymptotic to $\widehat{F}$, from the above analysis one is being chosen in a canonical way.

The canonical fundamental solution of $A$ on the sectors $\operatorname{Sect}_{j}$ are $\Phi_{j}:=\Sigma_{j}(\widehat{F}) u^{T_{1}} e^{Q}$, for a fixed $(\mathcal{A}, u, \widetilde{p})$ and $\left(A, g_{0}\right) \in \widehat{\operatorname{Syst}}(\mathcal{A})$, where for a fixed $p \in \operatorname{Sect}_{0}$ one has chosen a branch of $\log (u)$ giving a lift $\widetilde{p}$ of $p$ to the universal cover of the punctured disc $\mathbb{D}_{0}$. For this fixed data the Stokes factors are constant invertible matrices ${ }^{14}$

$$
K_{j}=e^{-Q} u^{-T_{1}} \kappa_{j} u^{T_{1}} e^{Q} \in \mathbb{S t o}_{\mathrm{d}_{j}}(\mathcal{A}),
$$

\footnotetext{
${ }^{14}$ Since $u^{T_{1}} e^{Q}$ is a fundamental solution of $\mathcal{A}$ (i.e. its columns are a basis of solutions) we have $d\left(K_{i}\right)=0$.
} 


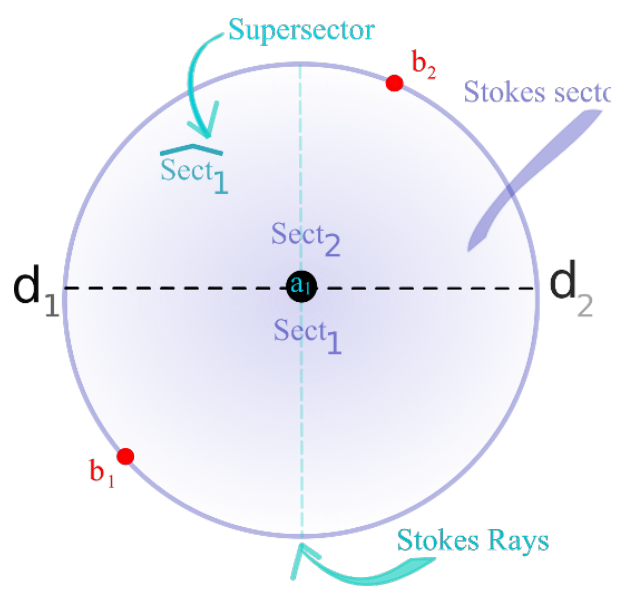

Figure 5. Data needed to define a tentacle.

where the $\kappa_{j}$ are matrix of holomorphic functions defined by

$$
\kappa_{j}=\Sigma_{j}(\widehat{F})^{-1} \circ \Sigma_{j-1}(\widehat{F})
$$

Remark A.11 Note that $\kappa_{j}[\mathcal{A}]=\mathcal{A}$, and thus it is an automorphism of $\mathcal{A}$. Moreover, $K_{6} \cdots K_{1}=P S_{6} \cdots S_{1} P^{-1}$, where $P$ is the identity matrix when the ordering is $q_{1}<q_{2}$.

If $\Phi_{j}$ is continued across the anti-Stokes ray $\mathrm{d}_{j+1}$, then on the sector Sect $_{j+1}$ it follows that $K_{j+1}:=\Phi_{j+1}^{-1} \circ \Phi_{j}$ for all $j$ except $K_{1}:=\Phi_{j+1}^{-1} \circ \Phi_{j} \circ M_{0}^{-1}$ for $i=6$, where as in Definition A.1 one has that $M_{0}:=e^{2 \pi i T_{1}}$ is the so-called formal monodromy.

The Stokes matrices $S_{j}=K_{j} K_{2(j-1)}$ are essentially the transition matrices between the canonical fundamental solutions: if $\Phi_{j}$ on Sect $_{j}$ is continued onto Sect ${ }_{j+1}$, then for $j=1, \ldots, 5$ one has $\Phi_{j}=\Phi_{j+1} \cdot P S_{j+1} P^{-1}$ where $P_{a b}=\delta_{c(a) b}$ for $c$ the permutation giving the ordering of the sector taken (so it's the identity matrix if we stick with the sector giving the ordering $\left.q_{1}<q_{2}\right)$. For $j=6$ one has that $\Phi_{6}=\Phi_{1} \cdot P S_{1} P^{-1} M_{0}$.

Remark A.12 If one continues $\Phi_{0}=\Phi_{6}$ around the sectors and back to Sect $t_{0}$, it will become

$$
\Phi_{0} \cdot P S_{6} \cdots S_{2} S_{1} P^{-1} M_{0}
$$

If a wild Higgs bundle had multiple poles, one of which of order 4, then the extra data needed to define a tentacle coming from the order 4 pole would be as in red in figure 5 .

Rank 2 Higgs bundles with 2 poles of order 2. When considering Higgs bundles with multiple poles of different orders, one needs to have a coherent way of encoding the data coming from each pole. Following [56], we shall recall here how this is done through the so-called tentacles in the case of a rank 2 Higgs bundles with exactly two poles of order 2 .

In a more general setting, consider Higgs bundles with different poles over a divisor $D=\left\{p_{1}, \ldots, p_{4}\right\}$ consisting of at most four points on $\mathbb{P}^{1}$, and let $V$ be a homomorphically trivial bundle over $\mathbb{P}^{1}$. Then, we may consider $u$ a local coordinate which vanishes at $p_{i}$, 


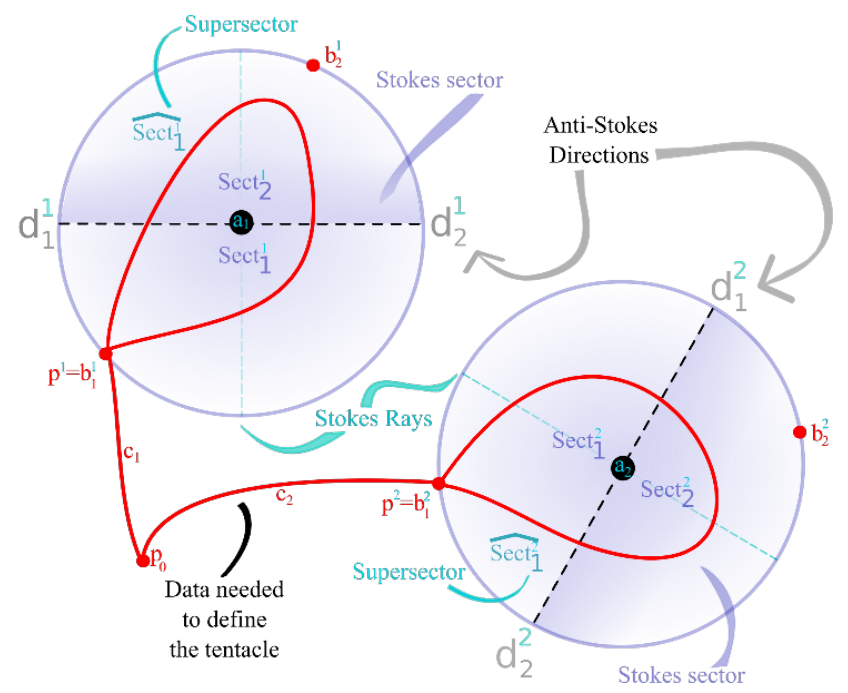

Figure 6. Tentacles for two poles of order 2.

and near each point $p_{i}$ there is a local trivialization of $V$. Then, near each $p_{i}$ let $\nabla=d-\mathcal{A}$ be a meromorphic connection with poles on each $p_{i} \in D$ of order $k_{i}$ : one may write

$$
\mathcal{A}=\sum_{i=1}^{4} d Q_{i}+T_{1}^{i} \frac{d u}{\left(u-p_{i}\right)}+\text { holomorphic terms }
$$

or equivalently, following the notation of section 4 , as

$$
\mathcal{A}=\sum_{i=1}^{4}\left(\frac{T_{k_{i}}^{i}}{\left(u-p_{i}\right)^{k_{i}}}+\cdots+\frac{T_{1}^{i}}{\left(u-p_{i}\right)^{1}}\right),
$$

where $T_{j}^{i}$ are $2 \times 2$ matrices of $(1,0)$ forms. As mentioned before, the connection $\nabla$ has a pole of order $k_{i}$ at $p_{i}$, and assuming that $T_{1}^{1}+\cdots+{ }_{1}^{4}=0$, it has no other poles. To deal with this type of Higgs bundles we choose disjoint open discs $\mathrm{d}_{i}$ on $\mathbb{P}^{1}$ with $p_{i} \in \mathrm{d}_{i}$ and, for each $i$, a coordinate $u_{i}$ on $\mathrm{d}_{i}$ vanishing at $p_{i}$. Thus the local picture above of the previous sections is repeated on each such disc.

Remark A.13 The Stokes data associated to each pole and its disc is put together through tentacles (see [56] where these were defined and thoroughly studied), and in what follows we shall review how these are obtained. In particular, one needs to fix a base point $p_{0} \neq p_{i}$, and a point in each sector.

Consider then a fixed base point $p_{0} \neq p_{i}$, and a choice $b_{\xi}$ in each sector bounded by anti-stokes directions at each point $p_{i}$. These points will allow one to define the tentacle associated to the Higgs bundle, which ultimately will consist of the data in figure 6 .

Define monodromy manifolds as spaces of representations of the groupoid $\widetilde{\Gamma}$ :

- The objects of $\widetilde{\Gamma}$ are the elements of $\widetilde{B}:=\left\{p_{0}\right\} \cup \widetilde{B}_{1} \cup \widetilde{B}_{2} \cup \widetilde{B}_{3} \cup \widetilde{B}_{4}$, where $\widetilde{B}_{i}$ is the discrete set of points of the universal cover of the punctured disc $\mathrm{d}_{i} / p_{i}$ which are above one of the $b_{\xi}$ 's. 
- If $\widetilde{p}_{1}, \widetilde{p}_{2} \in \widetilde{B}$, then the set of morphisms of $\widetilde{\Gamma}$ from $p_{1}$ to $p_{2}$ is the set of homotopy classes of paths $\gamma:[0,1] \rightarrow \mathbb{P}^{1}-\left\{p_{1}, p_{2}, p_{3}, p_{4}\right\}$ from $p_{1}$ to $p_{2}$. Here we have denoted by $p \in \mathbb{P}^{1}$ the underlying point of $\widetilde{p} \in \widetilde{B}$ (namely $p_{0}$ or one of the $b_{\xi}$ ).

For a path $\gamma_{p_{2} p_{1}}$ from $p_{1}$ to $p_{2}$, we shall denote by $\left[\gamma_{p_{2} p_{1}}\right] \in \widetilde{\Gamma}$ its class. Each choice of basis of the fibre $V_{p_{0}}$ of the rank 2 bundle $V$ at $p_{0}$, one has that $(V, \nabla)$ naturally determines a representation of the groupoid $\Gamma$ in the group $G=G L(2, \mathbb{C})$, as follows. Since there is a canonical choice of basis $\Phi: \mathbb{C}^{2} \rightarrow V$ of $\nabla$-horizontal sections of $V$ in a neighbourhood of $p_{i}$, for $i=1,2$, one can extend these bases along the path $\gamma_{p_{2} p_{1}}([0,1])$. Since they are both horizontal, one has that $\Phi_{1}=\Phi_{2} \cdot C$ on this path, for some $C \in G L(2, \mathbb{C})$. Then, the representation is $\rho\left(\left[\gamma_{p_{2} p_{1}}\right]\right):=C=\Phi_{2}^{-1} \Phi_{1}$. In particular, $\rho$ encodes all possible connection matrices between sectors at different poles as well as all the Stokes factors and Stokes matrices at each pole. Henceforth, we shall denote by $\gamma_{i}$ a path connecting $p_{0}$ to $p_{i}$, as appearing in red in figure 6 . The representations have two properties to which we shall allude in the coming sections (see [56, Lemma 3.6])

(SR1) For any $i$, if $p_{1} \in \widetilde{B}_{i}$ and $p_{2}$ is the next element of $B_{i}$ after $p_{1}$ in a positive sense, consider $\gamma_{p_{1} p_{2}}$ a small arc in $\mathrm{d}_{i}$ from $p_{1}$ to $p_{2}$. Then $\rho\left(\gamma_{p_{2} p_{1}}\right) \in \mathbb{S} t o_{d}\left(A_{i}^{0}\right)$, where $d$ is the unique anti-Stokes ray that $\gamma_{p_{2} p_{1}}$ crosses.

(SR2) For each $j$ there is a diagonal matrix $\Lambda_{j}$ (with distinct eigenvalues $\bmod \mathbb{Z}$ if $k_{j}=1$ ) such that for any $p_{1} \in \widetilde{B}_{j}, p_{2} \in \widetilde{B}$ and morphism $\gamma_{p_{2} p_{1}}$, one has that $\rho\left(\gamma_{p_{2}\left(p_{1}+2 \pi\right)}\right)=$ $\rho\left(\gamma_{p_{2} p_{1}}\right) \cdot e^{2 \pi i{ }^{j} \Lambda}$, where $\gamma_{p_{2}\left(p_{1}+2 \pi\right)}=\gamma_{p_{2} p_{1}}$ are equal as paths, but $\left(\widetilde{p}_{1}+2 \pi\right)$ is the next point of $\widetilde{B}_{j}$ after $\widetilde{p}_{1}$ (in a positive sense) which is also above $p_{1}$.

Definition A.14 A Stokes representation $\rho$ is a representation of the groupoid $\widetilde{\Gamma}$ into $\mathrm{GL}(2, \mathbb{C})$ together with a choice of 4 diagonal matrices ${ }^{j} \Lambda$ such that (SR1) and (SR2) hold. The set of Stokes representations will be denoted $\operatorname{Hom}_{\mathbb{S}}(\widetilde{\Gamma}, \operatorname{GL}(2, \mathbb{C}))$.

Definition A.15 The matrices ${ }^{j} \Lambda$ associated to a Stokes representation $\rho$ will be called the exponents of the formal monodromy of $\rho$ and the number $\operatorname{deg}(\rho):=\sum_{j} \operatorname{Tr}\left({ }^{j} \Lambda\right)$ is the degree of $\rho$.

In order to define the Stokes matrices in the case of multiples poles (through a definition consistent with (A.34)), one needs to make choices of points and paths between them. These choices are encoded in a choice of the so-called tentacle appearing in figure 6 :

1. A point $p_{j}$ in some sector at $p_{j}$ between two anti-Stokes rays, for $j=1, \ldots, 4$.

2. A lift $\tilde{p}_{j}$ of each $p_{j}$ to the universal cover of the punctured disc $\mathrm{d}_{j} /\left\{p_{i}\right\}$.

3. A base-point $p_{0} \in \mathbb{P}^{1} /\left\{p_{1}, \ldots, p_{4}\right\}$.

4. A path $\gamma_{j}:[0,1] \rightarrow \mathbb{P}^{1} /\left\{p_{1}, \ldots, p_{4}\right\}$ in the punctured sphere, from $p_{0}$ to $p_{j}$ for each $j$, such that the loop $\left(\gamma_{4}^{-1} \beta_{4} \gamma_{4}\right)\left(\gamma_{3}^{-1} \beta_{3} \gamma_{3}\right)\left(\gamma_{2}^{-1} \beta_{2} \gamma_{2}\right)\left(\gamma_{1}^{-1} \beta_{1} \gamma_{1}\right)$ based at $p_{0}$ is contractible in $\mathbb{P}^{1} /\left\{p_{1}, \ldots, p_{4}\right\}$, where $\beta_{j}$ is any loop in $\mathrm{d}_{j} /\left\{p_{j}\right\}$ based at $p_{j}$ encircling $p_{j}$ once in a positive sense. 
Remark A.16 Note that a choice of tentacle implies a labelling $P_{j}$ of the permutation associated to $p_{j}$ (which for simple poles is the identity matrix).

In the case of only one pole, one can still define the tentacle data associated to the system, which is consistent with what was described in previous sections. We shall denote by $C_{j}:=P_{j}^{-1} \rho\left(\gamma_{j}\right) \in \mathrm{GL}(2, \mathbb{C})$, for $j=1,2,3,4$, where as before $\gamma_{j}$ is the path connecting $p_{0}$ to $p_{j}$. Moreover, denote by ${ }^{i} \sigma_{j}$ the morphism from ${ }^{i} \widetilde{b}_{(j-1) \cdot l}$ to ${ }^{i} \widetilde{b}_{j \cdot l}$ with underlying path a simple arc in $\mathrm{d}_{i} /\left\{p_{i}\right\}$ from ${ }^{i} b_{(j-1) \cdot l}$ to ${ }^{i} b_{j \cdot l}$ in a positive sense (where $l=l_{i}=r_{i} /\left(2 k_{i}-2\right)$ ).

One defines the Stokes matrices by the formulae (which agrees with the case of just one pole in (A.34)):

$$
{ }^{i} S_{1}:=P_{i}^{-1} \rho\left({ }^{i} \sigma_{0}\right){ }^{i} M_{0}^{-1} P_{i} \text {. and }{ }^{i} S_{j}:=P_{i}^{-1} \rho\left({ }^{i} \sigma_{j}\right) P_{i} \text { for } j=2, \ldots, 2 k_{i}-2
$$

In the case of a rank 2 Higgs bundles with two poles of order two as we are considering in this section, there are only two marked points $p_{1}$ and $p_{2}$ and

$$
\nabla:=d-\left(T_{2}^{1} \frac{d u}{\left(u-p_{1}\right)^{2}}+T_{1}^{1} \frac{d u}{\left(u-p_{1}\right)}\right)-\left(T_{2}^{2} \frac{d u}{\left(u-p_{2}\right)^{2}}+T_{1}^{2} \frac{d u}{\left(u-p_{2}\right)}\right) .
$$

In this case one considers two discs $\mathrm{d}_{i}$ containing $p_{i}$, for $i=1,2$ and choose $p_{0} \in \mathbb{P}^{1}-\left\{p_{1}, p_{2}\right\}$ and $b_{j}^{i}$, for $i, j=1,2$, marked points in each of the two sectors that each disc $\mathrm{d}_{i}$ has. Then, morphisms of $\widetilde{\Gamma}$ correspond to paths connecting two points in $\left\{b_{j}^{i}, p_{0}\right\}_{i, j=1,2}$ not passing through $p_{1}, p_{2}$, and the tentacle is given by the data appearing in figure 6 .

Remark A.17 One should note that in this case the tentacle is determined by the choice of the discs $\mathrm{d}_{1}, \mathrm{~d}_{2}$, together with a base point $p_{0}$, two points $\mathrm{p}^{i}=\mathrm{b}_{1}^{i}$ and paths $\mathrm{c}_{i}$ from $\mathrm{p}_{0}$ to $\mathrm{p}_{i}$. Taking the canonical orientation the matrices $P_{i}$ yields the $2 \times 2$ identity matrices.

\section{B An integrable system}

In this section, we collect a few results from [68] which will make the argument leading to (4.53) more precise. The argument is a straightforward extension of an argument in [44], which also referred to [68], but not explicitly. We primarily work in terms of a gauge group $\mathrm{U}(N)$ and its complexification $\mathrm{GL}(N, \mathbb{C})$ so we state those first, then adapt them to $\operatorname{SL}(N, \mathbb{C})$.

We consider Higgs bundles on $C$ valued in $L=K_{C}(D)$, where $D$ is an effective divisor. So we have a rank $N$ and degree $d$ vector bundle $E$ on $C$ and a Higgs bundle structure

$$
\Phi: E \rightarrow E \otimes K_{C}(D) .
$$

If $D=\sum m_{i} p_{i}$, then $\Phi$ can be identified with a Higgs field having poles of order at most $m_{i}$ at $p_{i}$. Let

$$
B_{L}=H^{0}\left(C, K_{C}\right) \oplus H^{0}\left(C, K_{C}^{2}\right) \oplus \ldots H^{0}\left(C, K_{C}^{r}\right) .
$$

The characteristic polynomial of $\Phi$ gives rise to a map $\operatorname{Higgs}_{C}(N, d, L) \rightarrow B_{L}$, where $\operatorname{Higgs}_{C}(N, d, L)$ is the moduli space of Higgs bundles of the above type. We can define spectral covers for $L$-valued Higgs fields in perfect analogy to the case $D=0$ of the Hitchin system. 
Theorem. Suppose that $L^{\otimes N}$ is very ample and $\operatorname{deg}(D)>\max (2, \rho)$, where $0 \leq \rho<r$ is the residue of $d \bmod N$. Then

1. The moduli space $M_{C}(N, d, L)$ of stable $L$ - valued Higgs fields of rank $N$ and degree $d$ has a smooth component $M_{C}^{\mathrm{sm}}(N, d, L)$ of top dimension $N^{2}(2 g-2+\operatorname{deg}(D))+$ $1+\epsilon_{D}$, where $\epsilon_{D}$ is 1 if $D=0$ and zero if $D>0$. Furthermore, $M_{C}^{\mathrm{sm}}(N, d, L)$ is the unique component containing those Higgs pairs which are supported on irreducible and reduced spectral curves.

2. $M_{C}^{\mathrm{sm}}(N, d, L)$ has a canonical Poisson structure (depending on $\left.D\right)$.

3. The characteristic polynomial map $H: M_{C}^{\mathrm{sm}}(N, d, L) \rightarrow B_{L}$ is an algebraically completely integrable Hamiltonian system. The generic (Lagrangian) fiber is a complete Jacobian of a smooth spectral curve of genus $N^{2}(g-1)+1+(\operatorname{deg} D) N(N-1) / 2$.

All dimensions in the theorem are complex. Observe that the dimension of the fiber is computed readily by Riemann-Hurwitz for the spectral cover. The dimension of the base is computed by Riemann-Roch, and then the dimension of the total space is found by adding the dimensions of the base and fiber.

Similar results appear in [69], which also contains results for bundles with fixed determinant, i.e. $\operatorname{SL}(N, \mathbb{C})$-bundles. In this case, the base is replaced by

$$
B_{L}=H^{0}\left(C, K_{C}^{2}\right) \oplus H^{0}\left(C, K_{C}^{3}\right) \oplus \ldots H^{0}\left(C, K_{C}^{N}\right),
$$

and the fiber is replaced by a torsor for the Prym variety. Since we are interested in poles, we assume that $D \neq 0$. The dimension of the base is computed to be

$$
\left(N^{2}-1\right)(g-1)+\frac{(N+2)(N-1)}{2} \operatorname{deg}(D) .
$$

By subtracting $g$ from the dimension of the Jacobian, the dimension of the Prym is

$$
\left(N^{2}-1\right)(g-1)+\frac{(N(N-1)}{2} \operatorname{deg}(D),
$$

from which the dimension of the component of the moduli space is

$$
\left(N^{2}-1\right)(2 g-2+\operatorname{dim}(D)) .
$$

Specializing to $\mathbb{P}^{1}$, these can be summarized as the following theorem, where we wrote $M_{\mathbb{P}^{1}, 0}(N, L)$ for the moduli space of $\operatorname{SL}(N, \mathbb{C})$ Higgs bundles on $\mathbb{P}^{1}$ valued in $L$. Since $d=0$, the only condition is that $L$ is very ample, or equivalently $\operatorname{deg}(D) \geq 3$.

Theorem. Suppose that $\operatorname{deg}(D) \geq 3$. Then

1. The moduli space $M_{\mathbb{P}^{1}, 0}(N, L)$ of stable $L$ - valued $\operatorname{SL}(N, \mathbb{C})$ Higgs bundles has a smooth component $M_{\mathbb{P}^{1}, 0}^{\mathrm{sm}}(N, L)$ of top dimension $\left(N^{2}-1\right)(2 g-2+\operatorname{dim}(D))$.

2. The characteristic polynomial map $H: M_{C}^{\mathrm{sm}}(N, d, L) \rightarrow B_{L}$ is an algebraically completely integrable Hamiltonian system. The generic (Lagrangian) fiber is a torsor for the Prym variety of a smooth spectral cover, an abelian variety of dimension $\left(N^{2}-1\right)(g-1)+\frac{(N(N-1)}{2} \operatorname{deg}(D)$. 


\section{Complex structure deformations}

In this appendix, we describe complex structure deformations, extending the analysis in [4]. We implicitly make reference to these features when we translate background values for hypermultiplets to complex structure deformations.

We begin by recalling the relevant tools of deformation theory of a possibly singular algebraic variety $X$. Let $\Omega_{X}^{1}$ be the sheaf of holomorphic 1-forms on $X$. Then we put

$$
\mathcal{T}_{X}^{0}=\underline{\operatorname{Hom}}_{X}\left(\Omega_{X}^{1}, \mathcal{O}_{X}\right), \quad \mathcal{T}_{X}^{1}=\underline{\operatorname{Ext}}_{X}^{1}\left(\Omega_{X}^{1}, \mathcal{O}_{X}\right) .
$$

Then $\mathcal{T}_{X}^{0}$ is just the tangent sheaf of $X$, which is a vector bundle if $X$ is smooth but not in general. Furthermore, if $X$ is smooth, then $\mathcal{T}_{X}^{1}$ is zero because $\Omega_{X}^{1}$ is a vector bundle. Since all Ext sheaves are defined locally on $X$, we see that $\mathcal{T}_{X}^{1}$ is supported on the singular locus $\operatorname{Sing}(X)$ of $X$.

The first order deformations of $X$ are given by $\operatorname{Ext}_{X}^{1}\left(\Omega^{1}, \mathcal{O}_{X}\right)$ (the Ext group, not the Ext sheaf). The local to global spectral sequence for $H^{p}\left(X, \underline{E x t_{X}^{q}}\left(\Omega_{X}^{1}, \mathcal{O}_{X}\right)\right) \Longrightarrow$ $\left.\operatorname{Ext}_{X}^{q}\left(\Omega_{X}^{1}, \mathcal{O}_{X}\right)\right)$ gives

$$
0 \rightarrow H^{1}\left(X, \mathcal{T}_{X}^{0}\right) \rightarrow \operatorname{Ext}_{X}^{1}\left(\Omega_{X}^{1}, \mathcal{O}_{X}\right) \rightarrow H^{0}\left(X, \mathcal{T}_{X}^{1}\right) \stackrel{\delta}{\rightarrow} H^{2}\left(X, \mathcal{T}_{X}^{0}\right)
$$

The map $\operatorname{Ext}_{X}^{1}\left(\Omega^{1}, \mathcal{O}_{X}\right) \rightarrow H^{0}\left(X, \mathcal{T}_{X}^{1}\right)$ takes a global first order deformation of $X$ to the associated local deformation of a neighborhood of the singularity. Thus $H^{1}\left(X, \mathcal{T}_{X}^{0}\right)$ is the space of first order deformations of $X$ which preserve $\operatorname{Sing}(X)$, and furthermore, (C.2) says that a first order deformation $\rho \in H^{0}\left(X, \mathcal{T}_{X}^{1}\right)$ of a neighborhood of $\operatorname{Sing}(X)$ extends to a first order deformation of all of $X$ if and only if $\delta(\rho)=0$.

For emphasis, the domain $H^{0}\left(X, \mathcal{T}^{1}\right)$ of the map $\delta$ depends only on a neighborhood of $\operatorname{Sing}(X)$, but since $\mathcal{T}_{X}^{0}$ is supported on all of $X, \delta$ depends on the global geometry of $X$, not just a neighborhood of $\operatorname{Sing}(X)$. We point out that this is a familiar situation in string theory and in geometry, appearing in a compact conifold transition [70-72].

Now suppose we have a curve $C$ of $A_{1}$ singularities in a Calabi-Yau threefold $X$, so that $\operatorname{Sing}(X)=C$. Since $\mathcal{T}_{X}^{1}$ is local, we pick a local model for $X$ to be given by the equation $x y=z^{2}$ in the total space $Y$ of the bundle $L_{1} \oplus L_{2} \oplus K_{C}$, where $L_{1} \otimes L_{2} \simeq K_{C}^{2}$. Letting $\mathcal{I}_{X} \subset \mathcal{O}_{Y}$ be the ideal sheaf of $X$, we have the exact sequence

$$
0 \rightarrow \mathcal{I}_{X} /\left.\mathcal{I}_{X}^{2} \stackrel{d}{\longrightarrow} \Omega_{Y}^{1}\right|_{X} \rightarrow \Omega_{X}^{1} \rightarrow 0 .
$$

Letting $\pi: Y \rightarrow C$ denote the projection, we can rewrite this as

$$
\left.\left.0 \rightarrow\left(\pi^{*}\left(K_{C}^{-2}\right)\right)\right|_{X} \stackrel{d}{\longrightarrow}\left(\pi^{*}\left(K_{C}\right) \oplus \pi^{*}\left(L_{1}^{*}\right) \oplus \pi^{*}\left(L_{2}^{*}\right) \oplus \pi^{*}\left(K_{C}^{*}\right)\right)\right|_{X} \rightarrow \Omega_{X}^{1} \rightarrow 0 .
$$

The components of $d$ are given by the components of $d f=x d y+y d x+2 z d z$, i.e. $(0, y, x, 2 z)$. So we compute $\underline{E x t}_{X}^{1}$ by dualizing $d$

$$
\left.\left.\left(\pi^{*}\left(K_{C}^{*}\right) \oplus \pi^{*}\left(L_{1}\right) \oplus \pi^{*}\left(L_{2}\right) \oplus \pi^{*}\left(K_{C}\right)\right)\right|_{X} \stackrel{d^{\mathrm{t}}}{\longrightarrow}\left(\pi^{*}\left(K_{C}^{2}\right)\right)\right|_{X} \rightarrow \underline{\operatorname{Ex}} t_{X}^{1}\left(\Omega_{X}^{1}, \mathcal{O}_{X}\right) \rightarrow 0
$$


i.e. $\operatorname{Ext}_{X}^{1}\left(\Omega_{X}^{1}, \mathcal{O}_{X}\right)$ is the cokernel of $d^{\mathrm{t}}$. But the map $d^{\mathrm{t}}$ imposes $x=y=z=0$ on the cokernel, in other words, just restricts $\left.\left(\pi^{*}\left(K_{C}^{2}\right)\right)\right|_{X}$ to $C$. Thus $\operatorname{Ext}_{X}^{1}\left(\Omega_{X}^{1}, \mathcal{O}_{X}\right)$ is just $K_{C}^{2}$, or more formally, $i_{*}\left(\mathcal{O}_{C}\left(K_{C}^{2}\right)\right.$, where $i: C \hookrightarrow X$ is the inclusion. So the local deformations are simply given by $H^{0}\left(C, K_{C}^{2}\right)$, the base of the $\mathrm{SU}(2)$ Hitchin system.

Next, we look at the $A_{n}$ case, with local model $x y=z^{N+1}$ in the total space of $L_{1} \oplus L_{2} \oplus K_{C}$, where $L_{1} \otimes L_{2} \simeq K_{C}^{N+1}$. Then (C.4) is replaced by

$$
\left.\left.0 \rightarrow\left(\pi^{*}\left(K_{C}^{-N+1}\right)\right)\right|_{X} \stackrel{d}{\longrightarrow}\left(\pi^{*}\left(K_{C}\right) \oplus \pi^{*}\left(L_{1}^{*}\right) \oplus \pi^{*}\left(L_{2}^{*}\right) \oplus \pi^{*}\left(K_{C}^{*}\right)\right)\right|_{X} \rightarrow \Omega_{X}^{1} \rightarrow 0 .
$$

with $d$ in components given by $\left(0, y, x,(N+1) z^{N}\right)$. Similarly, $\operatorname{Ext}_{X}^{1}\left(\Omega_{X}^{1}, \mathcal{O}_{X}\right)$ is now

$$
\left.\left.\left(\pi^{*}\left(K_{C}^{*}\right) \oplus \pi^{*}\left(L_{1}\right) \oplus \pi^{*}\left(L_{2}\right) \oplus \pi^{*}\left(K_{C}\right)\right)\right|_{X} \stackrel{d^{\mathrm{t}}}{\longrightarrow}\left(\pi^{*}\left(K_{C}^{N+1}\right)\right)\right|_{X} \rightarrow \underline{\operatorname{Ext}}{ }_{X}^{1}\left(\Omega_{X}^{1}, \mathcal{O}_{X}\right) \rightarrow 0,
$$

with the new $d$. So $\underline{\operatorname{Ext}}_{X}^{1}\left(\Omega_{X}^{1}, \mathcal{O}_{X}\right)$ is the restriction of $\pi^{*}\left(K_{C}^{N+1}\right)$ to the infinitesimal neighborhood $C_{N-1}$ of $C$ defined by $x=y=z^{N}=0$, an invertible sheaf on $C_{N-1} \cdot{ }^{15}$

Let $\mathcal{I} \subset \mathcal{O}_{C_{N-1}}$ be the ideal of $C$ in $C_{N-1}$, locally generated by $z$. We get a filtration of $\mathcal{O}_{C_{N-1}}$

$$
\mathcal{O}_{C_{N-1}}=\mathcal{I}^{0} \supset \mathcal{I}^{1} \supset \cdots \supset \mathcal{I}^{N-1} \supset \mathcal{I}^{N}=0
$$

and a corresponding filtration of $\left.\pi^{*}\left(K_{C}^{N+1}\right)\right|_{C_{N-1}}$

$$
\left.\left.\left.\pi^{*}\left(K_{C}^{N+1}\right)\right|_{C_{N-1}} \supset \mathcal{I}^{1} \pi^{*}\left(K_{C}^{N+1}\right)\right|_{C_{N-1}} \supset \cdots \supset \mathcal{I}^{N-1} \pi^{*}\left(K_{C}^{N+1}\right)\right|_{C_{N-1}} \supset 0 .
$$

Now $\mathcal{I} / \mathcal{I}^{2}$ is the conormal bundle of $C$, isomorphic to $K_{C}^{*}$. It follows that $\mathcal{I}^{k} / \mathcal{I}^{k+1} \simeq K_{C}^{-k}$ for $k \leq N-1$. We can therefore break up (C.9) into short exact sequences

$$
\left.\left.0 \rightarrow \mathcal{I}^{k+1} \pi^{*}\left(K_{C}^{N+1}\right)\right|_{C_{N-1}} \rightarrow \mathcal{I}^{k} \pi^{*}\left(K_{C}^{N+1}\right)\right|_{C_{N-1}} \rightarrow i_{*}\left(\mathcal{O}_{C}\left(K_{C}^{N+1-k}\right)\right) \rightarrow 0,
$$

where by convention we put $\mathcal{I}^{0} \pi^{*}\left(K_{C}^{N+1}\right)=\pi^{*}\left(K_{C}^{N+1}\right)$. Observe that (C.10) remains exact on global sections

$$
0 \rightarrow H^{0}\left(\left.\mathcal{I}^{k+1} \pi^{*}\left(K_{C}^{N+1}\right)\right|_{C_{N-1}}\right) \rightarrow H^{0}\left(\left.\mathcal{I}^{k} \pi^{*}\left(K_{C}^{N+1}\right)\right|_{C_{N-1}}\right) \rightarrow H^{0}\left(C, K_{C}^{N+1-k}\right) \rightarrow 0,
$$

so that $H^{0}\left(X, \mathcal{T}^{1}\right)$ is filtered by the $H^{0}\left(\left.\mathcal{I}^{k} \pi^{*}\left(K_{C}^{N+1}\right)\right|_{C_{N-1}}\right)$ with graded quotients $H^{0}\left(C, K_{C}^{N+1-k}\right)$, and is thus (noncanonically) isomorphic to the Hitchin base $\oplus_{k=2}^{N+1} H^{0}\left(C, K_{C}^{k}\right)$.

However, to match with gauge theory, we need a canonical isomorphism. For this, recall that $\pi^{*}\left(K_{C}\right)$ has a canonical $K_{C}$-valued section which we call $\lambda$, familiar from the Hitchin system. Using $\lambda$, we have a map

$$
\bigoplus_{k=2}^{N+1} H^{0}\left(C, K_{C}^{k}\right) \stackrel{\phi}{\rightarrow} H^{0}\left(C_{N-1}, \pi^{*}\left(K_{C}^{N+1}\right)\right),\left.\quad\left(\omega_{2}, \ldots, \omega_{N+1}\right) \mapsto\left(\sum_{k=2}^{N+1} \pi^{*} \omega_{k} \lambda^{N+1-k}\right)\right|_{C_{N-1}} .
$$

\footnotetext{
${ }^{15}$ In [4], this was described less precisely as a vector bundle on $C$.
} 
Considering successive quotients and taking the previous discussion into consideration, we see immediately that the canonical map $\phi$ is an isomorphism, thereby identifying $H^{0}\left(X, \mathcal{T}^{1}\right)$ with the base of the $\mathrm{SU}(N)$ Hitchin system in the local case. Compare to [23].

We now allow the $A_{N-1}$ singularity to enhance to $A_{N}$ at isolated points $p_{i}$. In F-theory, this corresponds to the transverse intersection of an $I_{1}$ curve with the $I_{N}$ locus $C$. This is the situation considered in [4], which we now review.

Let $D=\sum_{i=1}^{d} p_{i}$ be the corresponding divisor. Our local model is

$$
x y=z^{N+1}+w z^{N},
$$

where $w \in H^{0}(C, \mathcal{O}(D))$ is a section vanishing precisely at the $p_{i}$.

In [4], it was shown that $\mathcal{T}^{1}$ has a torsion subsheaf $\operatorname{Tors}\left(\mathcal{T}^{1}\right)$, a skyscraper sheaf supported on $D$, one-dimensional over each $p_{i}$. Then $\mathcal{T}^{1}$ is annihilated by the partial derivatives $x, y,(n+1) z^{N}+N w z^{N-1}, z^{N}$, and the torsion class is generated by $z^{N-1}$. So $\mathcal{T}^{1} / \operatorname{Tors}\left(\mathcal{T}^{1}\right)$ is annihilated by $x, y, z^{N}$. In other words, if we let $C_{N-1}$ denote the nonreduced curve with equation $x=y=z^{N}=0$ as in the $A_{N-1}$ case, then $\mathcal{T}^{1} / \operatorname{Tors}\left(\mathcal{T}^{1}\right)$ is the locally free sheaf associated to a line bundle on $C_{N-1}$.

We have the short exact sequence

$$
0 \rightarrow \operatorname{Tors}\left(\mathcal{T}^{1}\right) \rightarrow \mathcal{T}^{1} \rightarrow \mathcal{T}^{1} /\left(\operatorname{Tors}\left(\mathcal{T}^{1}\right)\right) \rightarrow 0
$$

Since $H^{1}\left(\operatorname{Tors}\left(\mathcal{T}^{1}\right)\right)=0,($ C.14) remains exact on global sections:

$$
0 \rightarrow H^{0}\left(X, \operatorname{Tors}\left(\mathcal{T}^{1}\right)\right) \rightarrow H^{0}\left(X, \mathcal{T}^{1}\right) \rightarrow H^{0}\left(X, \mathcal{T}^{1}\right) /\left(\operatorname{Tors}\left(\mathcal{T}^{1}\right)\right) \rightarrow 0 .
$$

Concretely, the deformations are described as

$$
x y=z^{N+1}+w z^{N}+\sum_{j=1}^{N} \omega_{j} z^{N-j},
$$

with $\omega_{j} \in H^{0}\left(C, K_{C}^{j}(D)\right)$. The correspondence $\omega_{j} \leftrightarrow \omega_{j} / w$ identifies global sections of $\mathcal{O}\left(K_{C}^{j}(D)\right)$ with meromorphic sections of $\mathcal{O}\left(K_{C}^{j}\right)$ with first order poles on $D$, and we will frequently make this identification without comment. Away from $D$, the $u^{N-1}$ term can be eliminated by shifting $u$, so only the residues of $\omega_{1}$ are true parameters. The invariant way to say this is

$$
\operatorname{Tors}\left(\mathcal{T}^{1}\right)=\left.\mathcal{O}\left(K_{C}(D)\right)\right|_{D}, \quad H^{0}\left(X, \operatorname{Tors}\left(\mathcal{T}^{1}\right)\right)=H^{0}\left(D,\left.\mathcal{O}\left(K_{C}(D)\right)\right|_{D}\right) .
$$

Comparing the above local description with the global description of the pure $A_{N-1}$ case, we see that $\mathcal{T}^{1} /\left(\operatorname{Tors}\left(\mathcal{T}^{1}\right)\right.$ is the invertible sheaf of sections of the line bundle $\left.\pi^{*}\left(K_{C}^{N}(D)\right)\right|_{C_{N-1}}$ on $C_{N-1}$. We filter $\mathcal{T}^{1} / \operatorname{Tors}\left(\mathcal{T}^{1}\right)$ using powers of $\mathcal{I}$ as before, use $\mathcal{I} / \mathcal{I}^{2} \simeq \mathcal{O}\left(K_{C}^{*}\right)$, then finally use the spectral cover description as in (C.12) to canonically identify

$$
H^{0}\left(C, \mathcal{T}^{1} /\left(\operatorname{Tors}\left(\mathcal{T}^{1}\right)\right)\right) \simeq \bigoplus_{j=2}^{N} H^{0}\left(C, K_{C}^{j}(D)\right),
$$

the base of a parabolic Hitchin system. 
We now identify these moduli with the Higgs branch of an $\mathrm{SU}(N)$ gauge theory. For now, we content ourselves with describing the system in holomorphic gauge, ignoring the gauge field. Stability will guarantee that a gauge field can be found which satisfies the D-term constraints after going to unitary gauge.

We consider a Higgs field $\Phi$, and hypermultiplets $\psi_{i} \oplus \psi_{i}^{c}$ localized at $p_{i}$, with $\psi_{i}$ in the $\mathbf{N}$ representation and $\psi_{i}^{c}$ in the $\overline{\mathbf{N}}$. Note that the $N \times N$ matrix $\psi_{i} \otimes \psi_{i}^{c}$ has rank less than or equal to 1 . The rank can only be zero if either $\psi_{i}$ or $\psi_{i}^{c}$ is zero. Otherwise, the rank is 1 and $\psi_{i} \otimes \psi_{i}^{c}$ is either a projection operator onto a one-dimensional subspace, or nilpotent. These two cases are distinguished by the non-vanishing or vanishing of $\operatorname{Tr}\left(\psi_{i} \otimes \psi_{i}^{c}\right)$. This trace appears in the identity

$$
\left\langle\left\langle\psi_{j}, \psi_{j}^{c}\right\rangle\right\rangle=\psi_{i} \otimes \psi_{i}^{c}-\frac{1}{N} \operatorname{Tr}\left(\psi_{i} \otimes \psi_{i}^{c}\right) I_{N},
$$

where $I_{N}$ is the $N \times N$ identity matrix.

Intrinsically we can identify $\operatorname{Tr}\left(\psi_{i} \otimes \psi_{i}^{c}\right)$ with the residue of a meromorphic 1-form at $p_{i}$, identifying it with the torsion deformations $H^{0}\left(X, \operatorname{Tors}\left(\mathcal{T}^{1}\right)\right)$. If on the other hand $\operatorname{Tr}\left(\psi_{i} \otimes \psi_{i}^{c}\right)=0$, then $\left\langle\left\langle\psi_{j}, \psi_{j}^{c}\right\rangle\right\rangle=\psi_{i} \otimes \psi_{i}^{c}$ itself has rank 1. In the rank 0 case we similarly have $\left\langle\left\langle\psi_{j}, \psi_{j}^{c}\right\rangle\right\rangle=\psi_{i} \otimes \psi_{i}^{c}(=0)$ and these cases can now be combined.

To describe the correspondence and allow non-trivial gauge bundles, we let $\mathcal{P}$ denote a principal $G:=\mathrm{SU}(N)$-bundle on $C$. Then $\Phi \in \Gamma\left(\operatorname{ad}(\mathcal{P}) \otimes K_{C}(D)\right), \psi_{i} \in\left(\mathcal{P} \times{ }_{G} \mathbf{N}\right)_{p_{i}}$, and $\left.\psi_{i}^{c} \in\left(\mathcal{P} \times_{G} \overline{\mathbf{N}}\right)\right|_{p_{i}}$. The poles of $\Phi$ at the $p_{i}$ will be explained presently.

Then the equation for $\Phi$ is

$$
\bar{\partial} \Phi=\sum_{j} \delta_{p_{j}}\left\langle\left\langle\psi_{j}, \psi_{j}^{c}\right\rangle\right\rangle
$$

Thus $\Phi$ can have first order poles at the $p_{j}$.

We can rephrase (C.20) by saying that $\Phi$ is meromorphic, with first order poles at the $p_{i}$ whose residues are determined by $\psi$ and $\psi^{c}$. This perspective will be useful in generalizations.

We now match to geometry. As mentioned above, the case $\operatorname{Tr}\left(\psi_{i} \otimes \psi_{i}^{c}\right) \neq 0$ corresponds to the torsion deformation at $p_{i}$. Modding out by the torsion deformations, we suppose that $\operatorname{Tr}\left(\left\langle\left\langle\psi_{i}, \psi_{i}^{c}\right\rangle\right\rangle\right)=0$ for all $i$, so that $\left\langle\left\langle\psi_{i}, \psi_{i}^{c}\right\rangle\right\rangle$ is nilpotent with square zero.

Multiplying by $w$ to clear the poles, we let $z \in \mathcal{O}\left(K_{C}\right)$ and consider the characteristic polynomial

$$
z^{n+1}+w \operatorname{det}(z I+\Phi)
$$

which is of the form

$$
z^{N+1}+w z^{N}+\sum_{j=2}^{N} \omega_{j} z^{N-j} .
$$

Note that all poles in the expansion of the determinant in (C.21) are simple since each $\left\langle\left\langle\psi_{j}, \psi_{j}^{c}\right\rangle\right\rangle$ has rank 1 . Hence, for $j \geq 2$, we have $\omega_{j} \in H^{0}\left(K_{C}^{j}(D)\right)$, a precise match with the right hand side of (C.16). 
Now we turn to an $A_{N-1}-A_{M-1}$ collision, with local equation

$$
x y=z^{2}+u^{N} v^{M} .
$$

We let the two component curves be $C_{1}$ (corresponding to $u=0$ ) and $C_{2}$ (corresponding to $v=0$ ), intersecting transversally at the point $p$ corresponding to $u=v=0$. As before, the sheaf $\mathcal{T}_{X}^{1}$ is a line bundle on the scheme $D$ locally defined by the partial derivatives, $x=y=z=u^{N-1} v^{M}=u^{N} v^{M-1}=0$. Then $D$ is given locally by $u^{N}=0$ along $C_{1}-p$ and by $v^{M}=0$ along $C_{2}-p$.

Then $u^{N-1} v^{M-1}$ is a torsion class at $p$ as it is annihilated by each of $x, y, z, u, v$. Write $D_{\mathrm{CM}}$ for the reduction of $D$ modulo this torsion class. Thus $D_{\mathrm{CM}}$ has the single local equation $u^{N-1} v^{M-1}=0$, showing that $D_{\mathrm{CM}}$ is the union of the two (not necessarily reduced) components $\left(C_{1}\right)_{N-1}$ and $\left(C_{2}\right)_{M-1}$. Then we have a short exact sequence

$$
0 \rightarrow \operatorname{Tors}\left(\mathcal{T}_{X}^{1}\right) \rightarrow \mathcal{T}_{X}^{1} \rightarrow \mathcal{L} \rightarrow 0
$$

for some line bundle $\mathcal{L}$ on $D_{\mathrm{CM}}$.

Combining the previous discussion with the argument in the case of a defect considered in [4], we have $\left.\mathcal{L}\right|_{\left(C_{1}\right)_{N-1}} \simeq \pi^{*}\left(K_{C_{1}}^{N}(M p)\right)$ and $\left.\mathcal{L}\right|_{\left(C_{2}\right)_{M-1}} \simeq \pi^{*}\left(K_{C_{2}}^{M}(N p)\right)$, where $\left(C_{1}\right)_{N-1}$ is the thickening of $C_{1}$ defined by $u^{N-1}=0$, and similarly $\left(C_{2}\right)_{M-1}$ is the thickening of $C_{2}$ defined by $v^{M-1}=0$.

By restriction of $\mathcal{L}$ to the components we get an exact sequence

$$
\left.\left.\left.0 \rightarrow \mathcal{L} \rightarrow \mathcal{L}\right|_{\left(C_{1}\right)_{N-1}} \oplus \mathcal{L}\right|_{\left(C_{2}\right)_{M-1}} \rightarrow \mathcal{L}\right|_{u^{N-1}=v^{M-1}=0} \rightarrow 0,
$$

where the term $\left.\mathcal{L}\right|_{u^{N-1}=v^{M-1}=0}$ enforces an identification of sections of $\left.\mathcal{L}\right|_{\left(C_{1}\right)_{N-1}}$ and $\left.\mathcal{L}\right|_{\left(C_{2}\right)_{M-1}}$ necessary to get a section of $\mathcal{L}$. This gives an exact sequence

$$
0 \rightarrow H^{0}(\mathcal{L}) \rightarrow H^{0}\left(\left.\mathcal{L}\right|_{\left(C_{1}\right)_{N-1}}\right) \oplus H^{0}\left(\left.\mathcal{L}\right|_{\left(C_{2}\right)_{M-1}}\right) \rightarrow H^{0}\left(\left.\mathcal{L}\right|_{u^{N-1}=v^{M-1}=0}\right) \rightarrow 0
$$

where the map on the right is seen to be surjective, either by explicit calculation or by showing $H^{1}(\mathcal{L})=0$.

Combining these calculations with the calculation in the pure $A_{N}$ case, we deduce a canonical isomorphism

$$
H^{0}\left(\left.\mathcal{L}\right|_{\left(C_{1}\right)_{N-1}}\right) \simeq H^{0}\left(K_{C_{1}}^{2}(M p)\right) \oplus \ldots \oplus H^{0}\left(K_{C_{1}}^{N}(M p)\right),
$$

and similarly for the restriction of $\mathcal{L}$ to $\left(C_{2}\right)_{M-1}$.

For simplicity, we assume that $C_{1}$ has genus $g_{1} \geq 2$ (otherwise we can assume that there are sufficiently many collisions to ensure a nontrivial Hitchin base). Then $H^{0}\left(\left.\mathcal{L}\right|_{\left(C_{1}\right)_{N-1}}\right)$ has dimension $\left(N^{2}-1\right)\left(g_{1}-1\right)+M(N-1)$. We get a similar result for the restriction to $\mathrm{C}_{2}$.

Since $H^{0}\left(\left.\mathcal{L}\right|_{u^{N-1}=v^{M-1}=0}\right)$ has dimension $(M-1)(N-1)$, we get $\operatorname{dim} H^{0}(\mathcal{L})=\left(N^{2}-\right.$ 1) $\left(g_{1}-1\right)+\left(M^{2}-1\right)\left(g_{2}-1\right)+M(N-1)+N(M-1)-(M-1)(N-1)$, or

$$
\operatorname{dim} H^{0}(\mathcal{L})=\left(N^{2}-1\right)\left(g_{1}-1\right)+\left(M^{2}-1\right)\left(g_{2}-1\right)+M N-1 .
$$


But $h^{0}\left(\mathcal{T}_{X}^{1}\right)=h^{0}\left(\mathcal{T}_{X}^{1} /\left(\operatorname{Tors} \mathcal{T}_{X}^{1}\right)\right)+h^{0}\left(\operatorname{Tors} \mathcal{T}_{X}^{1}\right)=h^{0}(\mathcal{L})+1$, and so

$$
h^{0}\left(\mathcal{T}_{X}^{1}\right)=\left(N^{2}-1\right)\left(g_{1}-1\right)+\left(M^{2}-1\right)\left(g_{2}-1\right)+M N .
$$

This is precisely the dimension of the Higgs branch of an $\mathrm{SU}(N) \times \mathrm{SU}(M)$ gauge theory with $g_{1} \mathrm{SU}(N)$ adjoints, $g_{2} \mathrm{SU}(M)$ adjoints, and a bifundamental.

\section{C.1 Unfolding $A_{2}$}

Let us illustrate some of these considerations in the special case where we unfold an $A_{2}$ singularity, i.e. $N=3$. We now suppose that the $A_{1}$ singularity is enhanced to $A_{2}$ at a divisor $D$ in $C$, where the points in $D$ can have multiplicity greater than 1 . In F-theory, this situation arises from a collision between an $I_{2}$ and $I_{1}$ divisor where the components meet at $D$, including multiplicities from tangencies.

For computing $\mathcal{T}^{1}$ we start with a local model and then sort out how these fit together globally. Near a point $p$ of multiplicity $m$, we can take the local model

$$
x y+z^{3}+t^{m} z^{2}=0 .
$$

Computing partial derivatives, the sheaf $\mathcal{T}^{1}$ is locally just $\mathcal{O}_{X} /\left(y, x, 3 z^{2}+\right.$ $\left.2 t^{m} z, t^{m-1} z^{2}\right)$. But the element $z \in \mathrm{T}_{X}^{1}$ is a torsion class supported at $p$ since it is annihilated by $x, y, z^{2}$, and $t^{2 m-1}$, which jointly vanish only at $p$. In addition, $z$ is annihilated by $z t^{m-1}$. Hence the torsion subsheaf of $\mathcal{T}_{X}^{1}$ at $p$ is isomorphic to $\mathcal{O} /\left(x, y, z^{2}, z t^{m-1}, t^{2 m-1}\right)$, which has dimension $3 m-2$. The quotient of $\mathcal{T}_{X}^{1}$ by the torsion is clearly $\mathcal{O} /(x, y, z)$, a line bundle on $C$. Globally this is

$$
0 \rightarrow \operatorname{Tors}\left(\mathcal{T}_{X}^{1}\right) \rightarrow \mathcal{T}_{X}^{1} \rightarrow \mathcal{O}_{C}\left(K_{C}^{2}(D)\right) \rightarrow 0,
$$

where the line bundle at the end is identified globally exactly as in [4]. If $D=\sum m_{i} p_{i}$, then $\operatorname{dim} H^{0}\left(\operatorname{Tors}\left(\mathcal{T}_{X}^{1}\right)\right)=\sum_{i}\left(3 m_{i}-2\right)$.

Now, these additional moduli may appear to be inconsistent with the rules for F-theory. We reconcile these two viewpoints by showing that these local deformations are not in the kernel of the map $\delta$ from (C.2), so do not extend to global deformations in F-theory.

We content ourselves with considering the case of simple enhancements in F-theory. ${ }^{16}$ Consider the F-theory model with $f$ and $g$ given by

$$
f=-2 h_{4+2 n}^{2}+u f_{8+3 n}+u^{2} f_{8+2 n}+O\left(u^{3}\right), \quad g=3 h_{4+2 n}^{3}-u f_{8+3 n} h_{4+2 n}+u^{2} g_{12+4 n}+O\left(u^{3}\right),
$$

generically an $I_{2}$ singularity. From the leading order behavior of the discriminant

$$
u^{2}\left(-9 f_{8+3 n}^{2} h_{4+2 n}^{2}+108 h_{4+2 n}^{3} g_{12+4 n}+108 h_{4+2 n}^{4} f_{8+2 n}\right)+O\left(u^{3}\right),
$$

we see that we have enhancements at the zeros of

$$
-9 f_{8+3 n}^{2}+108 h_{4+2 n} g_{12+4 n}+108 h_{4+2 n}^{2} f_{8+2 n},
$$

\footnotetext{
${ }^{16}$ We thank W. Taylor for suggesting that we do this calculation.
} 
a set of $16+6 n$ points, generically distinct. We also have antisymmetric matter at the zeros of $h_{4+2 n}$, but these are singlets and can be ignored.

We can deform away from this F-theory geometry by relaxing the constraints

$$
f_{8+4 n}=-3 h_{4+2 n}^{2}, g_{12+6 n}=2 h_{4+2 n}^{3}, g_{12+5 n}=-f_{8+3 n} h_{4+2 n} .
$$

We study the map from $\left(f_{8+4 n}, g_{12+6 n}, g_{12+5 n}\right)$ to the space $\operatorname{Ext}_{X}^{1}\left(\Omega_{X}^{1}, \mathcal{O}_{X}\right)$ of first order deformation, and then compose with the map $\operatorname{Ext}_{X}^{1}\left(\Omega_{X}^{1}, \mathcal{O}_{X}\right) \rightarrow H^{0}\left(X, \mathcal{T}_{X}^{1}\right)$ from (C.2) by restricting attention to a neighborhood of the singularity.

We shift coordinates to the singularity along $u=0$ by the change of coordinates $x \mapsto X+h_{4+2 n}$. Then the Weierstrass equation becomes

$$
y^{2}=X^{3}+3 h_{4+2 n} X^{2}+f_{8+3 n} u X+u^{2}\left(g_{12+4 n}+f_{8+2 n} h_{4+2 n}\right)+O\left(u^{3}\right) .
$$

The singularity is located at $y=X=u=0$, and is visibly an $A_{1}$ generically. The singularity is enhanced at that points where the discriminant $f_{8+3 n}^{2}-12 h_{4+2 n}\left(g_{12+4 n}+\right.$ $\left.f_{8+2 n} h_{4+2 n}\right)$ of the quadratic form $3 h_{4+2 n} X^{2}+f_{8+3 n} u X+u^{2}\left(g_{12+4 n}+f_{8+2 n} h_{4+2 n}\right)$ vanishes. Of course this is precisely the divisor $D$ of $16+6 n$ points discussed above.

The terms involving $\left(f_{8+4 n}, g_{12+6 n}, g_{12+5 n}\right)$ change the Weierstrass equation by

$$
f_{8+4 n}\left(X+h_{4+2 n}\right)+g_{12+6 n}+g_{12+5 n} u .
$$

Comparing with the discussion earlier in this section, we see that the terms $f_{8+4 n} X+$ $g_{12+5 n} u$ which vanish on the singularity must correspond to torsion deformations, while the other terms $f_{8+4 n} h_{4+2 n}+g_{12+6 n}$ correspond to $H^{0}\left(\mathcal{T}^{1} / \operatorname{Tors}\left(\mathcal{T}^{1}\right)\right) \simeq H^{0}\left(C, K_{C}^{2}(D)\right)$. However, since $D$ has degree $16+6 n$ and $C \simeq \mathbb{P}^{1}$, we have $\mathcal{O}\left(K_{C}^{2}(D)\right) \simeq \mathcal{O}_{\mathbb{P}^{1}}(12+6 n)$. This is as it must be, since $f_{8+4 n} h_{4+2 n}+g_{12+6 n}$ has degree $12+6 n$.

To probe $\left.\operatorname{Tors}\left(\mathcal{T}^{1}\right)\right)$, we require $f_{8+4 n} h_{4+2 n}+g_{12+6 n}=0$, i.e. $g_{12+6 n}=-f_{8+4 n} h_{4+2 n}$. Then the free moduli which map to $\operatorname{Tors}\left(\mathcal{T}^{1}\right)$ correspond to the deformations

$$
f_{8+4 n} X+g_{12+5 n} u \text {. }
$$

Let's study the kernel of the map sending the deformation $f_{8+4 n} X+g_{12+5 n} u$ to $H^{0}\left(X, \mathcal{T}^{1}\right)$. By the calculation of $\mathcal{T}^{1}$, the kernel is generated by the partial derivatives of $3 h_{4+2 n} X^{2}+$ $f_{8+3 n} u X+u^{2}\left(g_{12+4 n}+f_{8+2 n} h_{4+2 n}\right)$, i.e.

$$
6 h_{4+2 n} X+f_{8+3 n} u, \quad f_{8+3 n} X+2 u\left(g_{12+4 n}+f_{8+2 n} h_{4+2 n}\right) .
$$

So the deformations which map to zero are generated by these two polynomials, and we conclude by comparing degrees that the kernel is given by

$$
f_{8+4 n} X+g_{12+5 n} u=p_{4+2 n}\left(6 h_{4+2 n} X+f_{8+3 n} u\right)+q_{n}\left(f_{8+3 n} X+2 u\left(g_{12+4 n}+f_{8+2 n} h_{4+2 n}\right)\right)
$$

for arbitrary polynomials $p_{4+2 n}$ and $q_{n}$ of respective degrees $4+2 n$ and $n$. Furthermore, for generic moduli there are no redundancies in the expression (C.40). So the dimension of the kernel is

$$
(2 n+5)+(n+1)=3 n+6 .
$$


Since the space of the expressions $f_{8+4 n} X+g_{12+5 n} u$ has dimension $(4 n+9)+(5 n+13)=9 n+$ 22 , we conclude that the space of torsion deformations realized in our F-theory geometry is $(9 n+22)-(3 n+6)=6 n+16$. Since the space of torsion deformations is also $6 n+16$, the degree of $D$, we conclude that for generic moduli all torsion deformations are realized in F-theory.

The situation is different for non-generic deformations. Suppose that we specialize to a situation where $m$ of the $16+6 n$ enhancement points coalesce, leaving $16+6 n-m$ ordinary enhancement points. By the calculation above, we get a $(16+6 n-m)+(3 m-2)=$ $14+6 n+2 m$ dimensional space of torsion deformations. But the above calculation of the kernel remains unchanged. The only difference is that we have $m-1$ additional moduli for deforming a pole of order $m$ to $m$ isolated poles. So the space of torsion deformations realized in our F-theory geometry is at most $(6 n+16)+(m-1)=6 n+m+15$. Since $6 n+$ $m+15<6 n+2 m+14$ for $m>1$, we conclude that not all of the local torsion deformations associated with a pole of order $m$ can be realized in a compact F-theory geometry.

It would be interesting to understand the matter associated with the singularity (C.30) in type IIA, and what in the gauge theory might prevent some of these putative deformations from being realized in a compact F-theory geometry.

Open Access. This article is distributed under the terms of the Creative Commons Attribution License (CC-BY 4.0), which permits any use, distribution and reproduction in any medium, provided the original author(s) and source are credited.

\section{References}

[1] J.M. Maldacena, The large-N limit of superconformal field theories and supergravity, Int. J. Theor. Phys. 38 (1999) 1113 [hep-th/9711200] [InSPIRE].

[2] R. Donagi, S. Katz and E. Sharpe, Spectra of D-branes with Higgs vevs, Adv. Theor. Math. Phys. 8 (2004) 813 [hep-th/0309270] [INSPIRE].

[3] S. Cecotti, C. Cordova, J.J. Heckman and C. Vafa, T-branes and monodromy, JHEP 07 (2011) 030 [arXiv: 1010.5780] [INSPIRE].

[4] L.B. Anderson, J.J. Heckman and S. Katz, T-branes and geometry, JHEP 05 (2014) 080 [arXiv: 1310.1931] [INSPIRE].

[5] A. Collinucci and R. Savelli, T-branes as branes within branes, JHEP 09 (2015) 161 [arXiv: 1410.4178] [INSPIRE].

[6] A. Collinucci and R. Savelli, F-theory on singular spaces, JHEP 09 (2015) 100 [arXiv: 1410.4867] [INSPIRE].

[7] A. Collinucci, S. Giacomelli, R. Savelli and R. Valandro, T-branes through 3d mirror symmetry, JHEP 07 (2016) 093 [arXiv: 1603.00062] [INSPIRE].

[8] I. Bena, J. Blåbäck, R. Minasian and R. Savelli, There and back again: a T-brane's tale, JHEP 11 (2016) 179 [arXiv: 1608.01221] [INSPIRE].

[9] C.-C. Chiou, A.E. Faraggi, R. Tatar and W. Walters, T-branes and Yukawa couplings, JHEP 05 (2011) 023 [arXiv: 1101.2455] [INSPIRE].

[10] A. Font, L.E. Ibáñez, F. Marchesano and D. Regalado, Non-perturbative effects and Yukawa hierarchies in F-theory SU(5) unification, JHEP 03 (2013) 140 [Erratum ibid. 07 (2013) 036] [arXiv: 1211.6529] [INSPIRE]. 
[11] A. Font, F. Marchesano, D. Regalado and G. Zoccarato, Up-type quark masses in SU(5) F-theory models, JHEP 11 (2013) 125 [arXiv:1307.8089] [INSPIRE].

[12] F. Marchesano, D. Regalado and G. Zoccarato, Yukawa hierarchies at the point of $E_{8}$ in F-theory, JHEP 04 (2015) 179 [arXiv:1503.02683] [INSPIRE].

[13] M. Cicoli, F. Quevedo and R. Valandro, De Sitter from T-branes, JHEP 03 (2016) 141 [arXiv: 1512.04558] [inSPIRE].

[14] F. Marchesano and S. Schwieger, T-branes and $\alpha^{\prime}$-corrections, JHEP 11 (2016) 123 [arXiv: 1609.02799] [INSPIRE].

[15] J.M. Ashfaque, Monodromic T-branes and the $\mathrm{SO}(10)_{\mathrm{GUT}}$, arXiv:1701.05896 [INSPIRE].

[16] J.J. Heckman and C. Vafa, From F-theory GUTs to the LHC, arXiv:0809.3452 [INSPIRE].

[17] J.J. Heckman, Particle physics implications of F-theory, Ann. Rev. Nucl. Part. Sci. 60 (2010) 237 [arXiv:1001.0577] [INSPIRE].

[18] T. Weigand, Lectures on F-theory compactifications and model building, Class. Quant. Grav. 27 (2010) 214004 [arXiv: 1009.3497] [INSPIRE].

[19] A. Maharana and E. Palti, Models of particle physics from type IIB string theory and F-theory: a review, Int. J. Mod. Phys. A 28 (2013) 1330005 [arXiv:1212.0555] [InSPIRE].

[20] M. Wijnholt, Higgs bundles and string phenomenology, Proc. Symp. Pure Math. 85 (2012) 275 [arXiv: 1201.2520] [INSPIRE].

[21] N.J. Hitchin, The selfduality equations on a Riemann surface, Proc. Lond. Math. Soc. 55 (1987) 59 [INSPIRE].

[22] D.-E. Diaconescu, R. Donagi, R. Dijkgraaf, C. Hofman and T. Pantev, Geometric transitions and integrable systems, Nucl. Phys. B 752 (2006) 329 [hep-th/0506196] [INSPIRE].

[23] D.-E. Diaconescu, R. Donagi and T. Pantev, Intermediate Jacobians and ADE Hitchin systems, hep-th/0607159 [INSPIRE].

[24] C. Beasley, J.J. Heckman and C. Vafa, GUTs and exceptional branes in F-theory - I, JHEP 01 (2009) 058 [arXiv:0802.3391] [INSPIRE].

[25] N. Hitchin, Lie groups and Teichmüller theory, Topology 31 (1992) 449.

[26] S.H. Katz and C. Vafa, Matter from geometry, Nucl. Phys. B 497 (1997) 146 [hep-th/9606086] [INSPIRE].

[27] R. Donagi and M. Wijnholt, Higgs bundles and UV completion in F-theory, Commun. Math. Phys. 326 (2014) 287 [arXiv:0904.1218] [INSPIRE].

[28] H. Hayashi, T. Kawano, R. Tatar and T. Watari, Codimension-3 singularities and Yukawa couplings in F-theory, Nucl. Phys. B 823 (2009) 47 [arXiv:0901.4941] [INSPIRE].

[29] R. Donagi, L. Ein and R. Lazarsfeld, Nilpotent cones and sheaves on K3 surfaces, Contemp. Math. 207 (1997) 51.

[30] L.B. Anderson, J. Gray, A. Lukas and E. Palti, Two hundred heterotic standard models on smooth Calabi-Yau threefolds, Phys. Rev. D 84 (2011) 106005 [arXiv:1106.4804] [INSPIRE].

[31] L.B. Anderson, J. Gray, A. Lukas and B. Ovrut, Vacuum varieties, holomorphic bundles and complex structure stabilization in heterotic theories, JHEP 07 (2013) 017 [arXiv:1304.2704] [INSPIRE]. 
[32] L.B. Anderson, A. Constantin, S.-J. Lee and A. Lukas, Hypercharge flux in heterotic compactifications, Phys. Rev. D 91 (2015) 046008 [arXiv:1411.0034] [InSPIRE].

[33] M. Bershadsky, K.A. Intriligator, S. Kachru, D.R. Morrison, V. Sadov and C. Vafa, Geometric singularities and enhanced gauge symmetries, Nucl. Phys. B 481 (1996) 215 [hep-th/9605200] [INSPIRE].

[34] P.S. Aspinwall and R.Y. Donagi, The heterotic string, the tangent bundle and derived categories, Adv. Theor. Math. Phys. 2 (1998) 1041 [hep-th/9806094] [INSPIRE].

[35] M. Del Zotto, J.J. Heckman, A. Tomasiello and C. Vafa, $6 d$ conformal matter, JHEP 02 (2015) 054 [arXiv: 1407.6359] [INSPIRE].

[36] J.J. Heckman, More on the matter of 6D SCFTs, Phys. Lett. B 747 (2015) 73 [arXiv: 1408.0006] [INSPIRE].

[37] J.J. Heckman, T. Rudelius and A. Tomasiello, $6 D$ RG flows and nilpotent hierarchies, JHEP 07 (2016) 082 [arXiv: 1601.04078] [INSPIRE].

[38] N. Mekareeya, T. Rudelius and A. Tomasiello, T-branes, anomalies and moduli spaces in $6 \mathrm{D}$ SCFTs, arXiv: 1612.06399 [INSPIRE].

[39] D. Baraglia, M. Kamgarpour and R. Varma, Complete integrability of the parahoric Hitchin system, arXiv: 1608.05454.

[40] D.H. Collingwood and W.M. McGovern, Nilpotent orbits in semisimple Lie algebras, Van Nostrand Reinhold, New York U.S.A., (1993).

[41] S. Gukov and E. Witten, Gauge theory, ramification, and the geometric Langlands program, hep-th/0612073 [INSPIRE].

[42] M. Bershadsky, K.A. Intriligator, S. Kachru, D.R. Morrison, V. Sadov and C. Vafa, Geometric singularities and enhanced gauge symmetries, Nucl. Phys. B 481 (1996) 215 [hep-th/9605200] [INSPIRE].

[43] P. Boalch, Geometry of moduli spaces of meromorphic connections on curves, Stokes data, wild non-Abelian Hodge theory, hyper-Kähler manifolds, isomonodromic deformations, Painlevé equations and relations to Lie theory, thése d'habilitation, Université Paris XI, Paris France, (2013) [arXiv:1305.6593] [INSPIRE].

[44] E. Witten, Gauge theory and wild ramification, arXiv:0710.0631 [INSPIRE].

[45] M. Jimbo, T. Miwa and K. Ueno, Monodromy preserving deformations of linear differential equations with rational coefficients I, Physica D 2 (1981) 306.

[46] N. Marcus, A. Sagnotti and W. Siegel, Ten-dimensional supersymmetric Yang-Mills theory in terms of four-dimensional superfields, Nucl. Phys. B 224 (1983) 159 [INSPIRE].

[47] N. Arkani-Hamed, T. Gregoire and J.G. Wacker, Higher dimensional supersymmetry in $4 D$ superspace, JHEP 03 (2002) 055 [hep-th/0101233] [INSPIRE].

[48] R. Dijkgraaf and C. Vafa, A perturbative window into nonperturbative physics, hep-th/0208048 [INSPIRE].

[49] S. Cecotti, M.C.N. Cheng, J.J. Heckman and C. Vafa, Yukawa couplings in F-theory and non-commutative geometry, arXiv:0910.0477 [INSPIRE].

[50] R. Donagi and M. Wijnholt, Model building with F-theory, Adv. Theor. Math. Phys. 15 (2011) 1237 [arXiv:0802.2969] [INSPIRE]. 
[51] J. Fisher and S. Rayan, Hyperpolygons and Hitchin systems, Int. Math. Res. Not. 2016 (2016) 1839 [arXiv: 1410.6467] [INSPIRE].

[52] T. Hausel, M. Mereb and M. Lennox Wong, Arithmetic and representation theory of wild character varieties, arXiv:1604.03382.

[53] S. Rayan, The quiver at the bottom of the twisted nilpotent cone on $P^{1}$, Eur. J. Math. (2016) [arXiv: 1609.08226] [INSPIRE].

[54] L. Godinho and A. Mandini, Hyperpolygon spaces and moduli spaces of parabolic Higgs bundles, arXiv:1101.3241.

[55] R. Hartshorne, Algebraic geometry, Graduate texts in mathematics, Springer, New York U.S.A., (1977).

[56] P. Boalch, Symplectic manifolds and isomonodromic deformations, Adv. Math. 163 (2001) 137.

[57] O. Biquard and P. Boalch, Wild non-Abelian Hodge theory on curves, Compos. Math. 140 (2004) 179 [math.DG/0111098].

[58] A. Beauville, Jacobiennes des courbes spectral et systèmes Hamiltoniens complètement intégrables (in French), Acta Math. 164 (1990) 211.

[59] L.B. Anderson, J. Gray, N. Raghuram and W. Taylor, Matter in transition, JHEP 04 (2016) 080 [arXiv: 1512.05791] [INSPIRE].

[60] S. Bradlow and L. Schaposnik, Higgs bundles and exceptional isogenies, Res. Math. Sci. 3 (2016) 14 [arXiv: 1508.02650].

[61] D.R. Morrison and C. Vafa, Compactifications of F-theory on Calabi-Yau threefolds - II, Nucl. Phys. B 476 (1996) 437 [hep-th/9603161] [INSPIRE].

[62] L.P. Schaposnik, Spectral data for G-Higgs bundles, arXiv:1301.1981.

[63] N.J. Hitchin, Langlands duality and $G_{2}$ spectral curves, math/0611524 [INSPIRE].

[64] N.J. Hitchin, Stable bundles and integrable systems, Duke Math. J. 54 (1987) 91 [INSPIRE].

[65] P.P. Boalch, Geometry and braiding of Stokes data; fission and wild character varieties, Ann. Math. 179 (2014) 301 [arXiv:1111.6228].

[66] P. Boalch, Hyper-Kähler manifolds and non-Abelian Hodge theory of (irregular) curves, arXiv: 1203.6607

[67] J. Martinet and J.P. Ramis, Elementary acceleration and multisummability I, Ann. Inst. H. Poincaré Phys. Théor. 54 (1991) 331.

[68] R. Donagi and E. Markman, Spectral curves, algebraically completely integrable Hamiltonian systems and moduli of bundles, alg-geom/9507017 [INSPIRE].

[69] F. Bottacin, Symplectic geometry on moduli spaces of stable pairs, Ann. Sci. École Norm. Sup. 28 (1995) 391.

[70] H. Clemens, Double solids, Adv. Math. 47 (1983) 107 [INSPIRE].

[71] R. Friedman, Simultaneous resolution of threefold double points, Math. Ann. 274 (1986) 671.

[72] B.R. Greene, D.R. Morrison and A. Strominger, Black hole condensation and the unification of string vacua, Nucl. Phys. B 451 (1995) 109 [hep-th/9504145] [INSPIRE]. 